\title{
Mutation Testing Advances: An Analysis and Survey
}

\author{
Mike Papadakis ${ }^{\mathrm{a}}$, Marinos Kintis ${ }^{\mathrm{a}}$, Jie Zhang ${ }^{\mathrm{c}}$, Yue Jia ${ }^{\mathrm{b}}$, Yves Le Traon ${ }^{\mathrm{a}}$, \\ Mark Harman ${ }^{\mathrm{b}}$ \\ ${ }^{a}$ Luxembourg University \\ ${ }^{b}$ University College London \\ ${ }^{c}$ Peking University
}

\begin{abstract}
Mutation testing realises the idea of using artificial defects to support testing activities. Mutation is typically used as a way to evaluate the adequacy of test suites, to guide the generation of test cases and to support experimentation. Mutation has reached a maturity phase and gradually gains popularity both in academia and in industry. This chapter presents a survey of recent advances, over the past decade, related to the fundamental problems of mutation testing and sets out the challenges and open problems for the future development of the method. It also collects advices on best practices related to the use of mutation in empirical studies of software testing. Thus, giving the reader a 'mini-handbook'-style roadmap for the application of mutation testing as experimental methodology.
\end{abstract}

Keywords: Mutation Testing, Software Testing, Survey, Seeded Faults

\section{Introduction}

How can we generate test cases that reveal faults? How confident are we with our test suite? Mutation analysis answers these questions by checking the ability of our tests to reveal some artificial defects. In case our tests fail to reveal the

\footnotetext{
* Corresponding author

Email addresses: michail.papadakis@uni.lu (Mike Papadakis), marinos.kintis@uni.lu (Marinos Kintis), zhangjie_marina@pku.edu.cn (Jie Zhang), yue.jia@ucl.ac.uk (Yue Jia), yves.letraon@uni.lu (Yves Le Traon), mark.harman@ucl.ac.uk (Mark Harman)
}

Preprint submitted to Journal of ${ }^{A} T_{E} X$ Templates

December 14, 2017 
artificial defects, we should not be confident on our testing and should improve our test suites. Mutation realises this idea and measures the confidence inspired by our testing. This method has reached a maturity phase and gradually gains popularity both in academia and in industry [1. Figures 1 and 2 record the current trends on the number of publications according to the data we collected (will be presented later). As demonstrated by these figures, the number of scientific publications relying on mutation analysis is continuously increasing, demonstrated in Figure 1 and numerous of these contributions appear in the major software engineering venues, shown in Figure 2 Therefore, mutation testing can be considered as a mainstream line of research.

The underlying idea of mutation is to force developers to design tests that explore system behaviours that reveal the introduced defects. The diverge types of defects that one can use, leads to test cases with different properties as these are designed to reflect common programming mistakes, internal boundary conditions, hazardous programming constructs and emulate test objectives of other structural test criteria. Generally, the method is flexible enough that it can be adapted to check almost everything that developers want to check, i.e., by formulating appropriate defects.

The flexibility of mutation testing is one of the key characteristics that makes it popular and generally applicable. Thus, mutation has been used for almost all forms of testing. Its primary application level is unit testing but several advances have been made in order to support other levels, i.e., specification [2], design [3], integration [4] and system levels [5]. The method has been applied on the most popular programming languages such as $\mathrm{C}[6], \mathrm{C}++[7, \mathrm{C} \#[8$, Java 9, JavaScript [10, Ruby [1] including specification [2] and modelling languages [12. It has also been adapted for the most popular programming paradigms such as Object-Oriented [13], Functional [14], aspect-oriented and declarative-oriented [15, 16] programming.

In the majority of the research projects, mutation was used as an indicator of test effectiveness. However, recently researchers have also focused on a different aspect: the exploration of the mutant behaviours. Thus, instead of exploring 
the behaviour space of the program under analysis the interest shifts to the behaviour of the mutants. In this line of research, mutants are used to identify important behaviour differences that can be either functional or non-functional. By realising this idea, one can form methods that assist activities outside software testing. Examples of this line of research are methods that automatically localise faults [17, automatically repair software [18, automatically improve programs' non-functional properties such as security [19, memory consumption [20] and execution speed [20, 21].

Mutation analysis originated in early work in the 1970s [22 24], but has a long and continuous history of development improvement, with particularly important advances in breakthroughs in the last decade, which constitute the focus of this survey. Previous surveys can be traced back to the work of DeMillo 25] (in 1989), which summarised the early research achievements. Other surveys are due to Offutt and Untch [26 (in 2000) and Jia and Harman [27. (in 2011). Offutt and Untch summarised the history of the technique and listed the main problems and solutions at that time. Subsequently, Jia and Harman comprehensively surveyed research achievements up to the year 2009 .

There is also a number of specialised surveys on specific problems of mutation testing. These are: a survey on the Equivalent mutant problem, by Madeyski et al. 28] (in 2014), a systematic mapping of mutation-based test generation by Souza et al. [29] (in 2014), a survey on model-based mutation testing [30] (in 2016) and a systematic review on search-based mutation testing by Silva et al. [31] (in 2017). However, none of these covers the whole spectrum of advances from 2009. During these years there are many new developments, applications, techniques and advances in mutation testing theory and practice as witnessed by the number of papers we analyse (more than 400 papers). These form the focus of the present chapter.

Mutation testing is also increasingly used as a foundational experimental methodology in comparing testing techniques (whether or not these techniques are directly relevant to mutation testing itself). The past decade has also witnessed an increasing focus on the methodological soundness and threats to valid- 


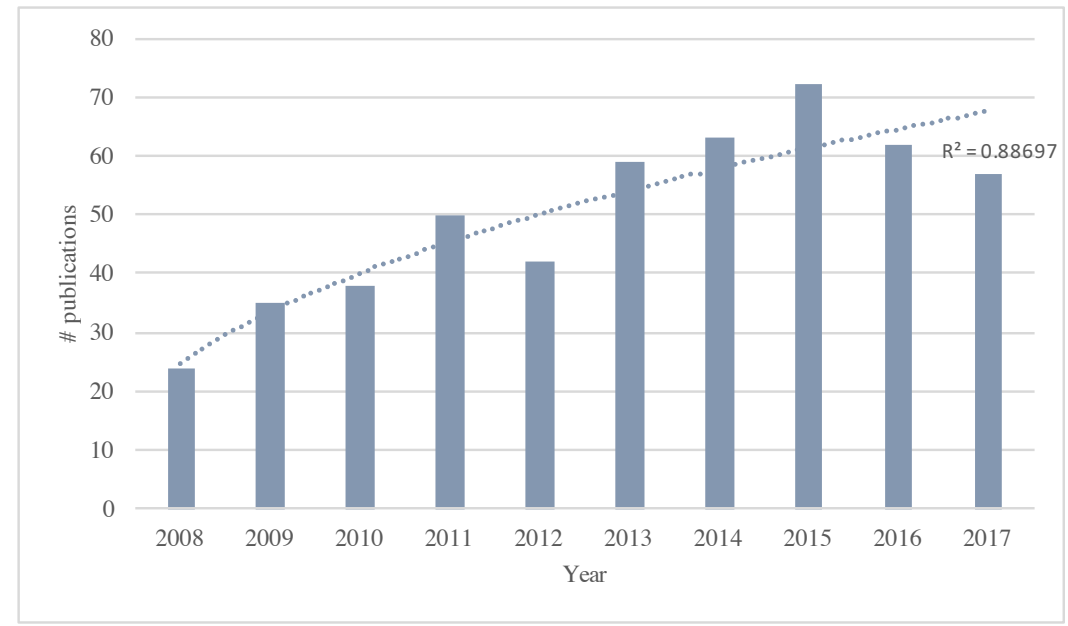

Figure 1: Number of mutation testing publications per year (years: 2008-2017).

ity that accrue from such use of mutation testing and the experimental methodology for wider empirical studies of software testing. Therefore, the present chapter also collects together advices on best practices, giving the reader a 'mini-handbook'-style roadmap for the application of mutation testing as an experimental methodology (in Section 9).

The present chapter surveys the advances related to mutation testing, i.e., using mutation analysis to detect faults. Thus, its focus is the techniques and studies that are related to mutation-guided test process. The goal is to provide a concise and easy to understand view of the advances that have been realised and how they can be used. To achieve this, we categorise and present the surveyed advances according to the stages of the mutation testing process that they apply to. In other words, we use the mutation testing process steps as a map for detailing the related advances. We believe that such an attempt will help readers, especially those new to mutation testing, understand everything they need in order to build modern mutation testing tools, understand the main challenges in the area and perform effective testing.

The survey was performed by collecting and analysing papers published in 


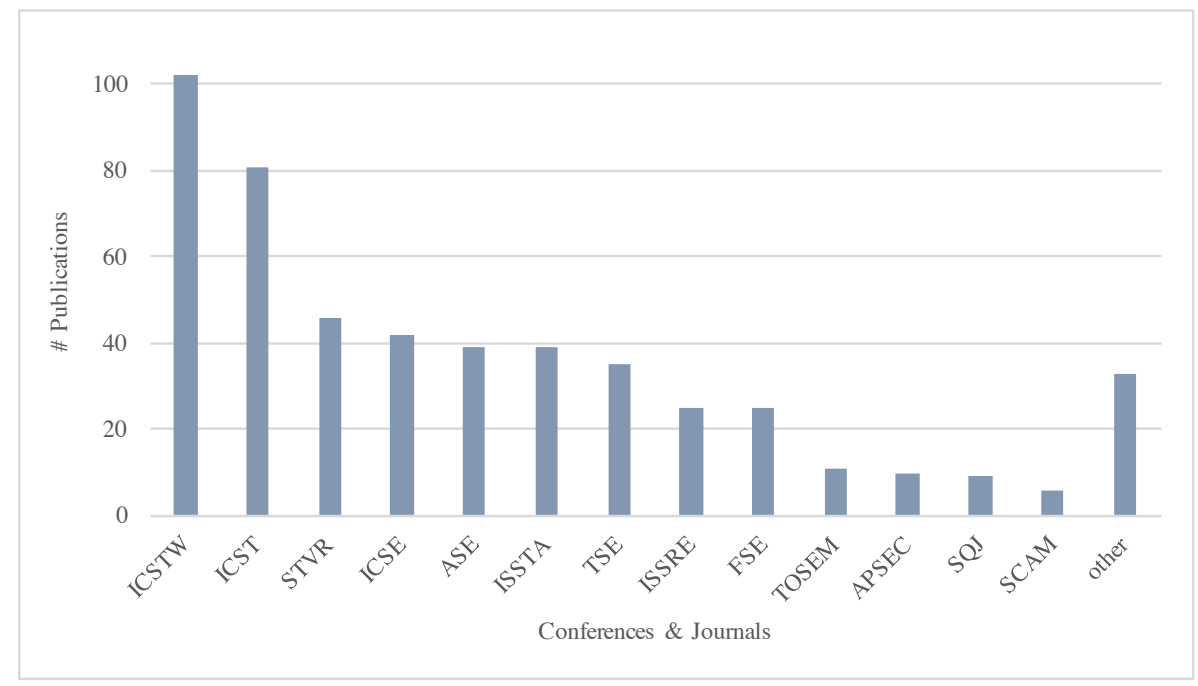

Figure 2: Number of mutation testing publications per scientific venue.

the last 10 years (2008-2017) in leading software engineering venues. This affords our survey a two-year overlap in the period covered with the previous survey of Jia and Harman [27. We adopted this approach to ensure that there is no chance that the paper could 'slip between the cracks' of the two surveys. Publication dating practices can differ between publishers, and there is a potential time lag between official publication date and the appearance of a paper, further compounded by the blurred distinction between online availability and formal publication date. Allowing this overlap lends our survey a coherent, decadewide, time window and also aims to ensure that mutation testing advances do not go uncovered due to such publication date uncertainties.

Thus, we selected papers published in the ICSE, SigSoft FSE, ASE, ISSTA, ICST, ICST Workshops (ICSTW), ISSRE, SCAM and APSEC conferences. We also collected papers published in the TOSEM, TSE, STVR and SQJ journals and formed our set of surveyed papers. We augmented this set with additional papers based on our knowledge. Overall, we selected a set of 502 papers, which fall within five generic categories, those that deal with the code-based mutation testing problems (186 papers), those concerning model-based mutation testing 
(40 papers), those that tackle problems unrelated to mutation testing problems (25 papers), those that describe mutation testing tools (34 papers) and those that use mutation testing only to perform test assessment (217 papers). In an attempt to provide a complete view of the fundamental advances in the area we also refer to some of the publications that were surveyed by the two previous surveys on the topic, i.e., the surveys of Offutt and Untch [26] and Jia and Harman [27, which have not been obviated by the recent research.

The rest of the chapter is organised as follows: Section 2 presents the main concepts that are used across the chapter. Sections 3 and 4 respectively motivate the use of mutation testing and discuss its relation with real faults. Next, the regular and other code-based advances are detailed in Sections 5 and 6 . Applications of mutation testing to other artefacts than code and a short description of mutation testing tools are presented in Sections 7 and 8 . Sections 9 and 10 present issues and best practices for using mutation testing in experimental studies. Finally, Section 11 concludes the chapter and outlines future research directions.

\section{Background}

Mutation analysis refers to the process of automatically mutating the program syntax with the aim of producing semantic program variants, i.e., generating artificial defects. These programs with defects (variants) are called mutants. Some mutants are syntactically illegal, e.g., cannot compile, named 'stillborn' mutants, and have to be removed. Mutation testing refers to the process of using mutation analysis to support testing by quantifying the test suite strengths. In the testing context, mutants form the objectives of the test process. Thus, test cases that are capable of distinguishing the behaviours of the mutant programs from those of the original program fulfil the test objectives. When a test distinguishes the behaviour of a mutant (from that of the original program) we say that the mutant is 'killed' or 'detected'; in a different case, we say that the the mutant is 'live'. 
Depending on what we define as program behaviour we can have different mutant killing conditions. Typically, what we monitor are all the observable program outputs against each running test: everything that the program prints to the standard/error outputs or is asserted by the program assertions. A mutant is said to be killed weakly [32, if the program state immediately after the execution of the mutant differs from the one that corresponds to the original program. We can also place the program state comparison at a later point after the execution of a mutant. This variation is called firm mutation [32]. A mutant is strongly killed if the original program and the mutant exhibit some observable difference in their outputs. Thus, we have variants of mutation, called weak, firm and strong. Overall, for weak/firm mutation, the condition of killing a mutant is that the program state has to be changed, while the changed state does not necessarily need to propagate to the output (as required by strong mutation). Therefore, weak mutation is expected to be less effective than firm mutation, which is in turn less effective than strong mutation. However, due to failed error propagation (subsequent computations may mask the state differences introduce by the mutants) there is no formal subsumption relation between any of the variants 32 .

Mutants are generated by altering the syntax of the program. Thus, we have syntactic transformation rules, called 'mutant operators', that define how to introduce syntactic changes to the program. For instance, an arithmetic mutant operator alters the arithmetic programming-language operator, changing + to - , for example. A basic set of mutant operators, which is usually considered as a minimum standard for mutation testing 33 is the five-operator set proposed by Offutt et al. 34. This set includes the relational (denoted as ROR), logical (denoted as LCR), arithmetic (denoted as AOR), absolute (denoted as ABS) and unary insertion (denoted as UOI) operators. Table 1 summarises these operators.

Defining mutant operators is somehow easy, we only need to define some syntactic alterations. However, defining useful operators is generally challenging. Previous research followed the path of defining a large set (almost exhaustive) 
Table 1: The popular five-operator set (proposed by Offutt et al. 34])

\begin{tabular}{|c|c|c|}
\hline Names & Description & Specific mutation operator \\
\hline $\mathrm{ABS}$ & Absolute Value Insertion & $\{(e, 0),(e, \operatorname{abs}(e)),(e,-\operatorname{abs}(e))\}$ \\
\hline AOR & Arithmetic Operator Replacement & $\begin{array}{l}\{((\mathrm{a} \text { op b }), \quad a),((\mathrm{a} \text { op b }), \quad b),(x, y) \mid x, y \in \\
\{+,-, *, /, \%\} \wedge x \neq y\}\end{array}$ \\
\hline $\mathrm{LCR}$ & Logical Connector Replacement & $\begin{array}{l}\{((\mathrm{a} o p \mathrm{~b}), a),((\mathrm{a} o p \mathrm{~b}), b),((\mathrm{a} o p \mathrm{~b}), \text { false }),((\mathrm{a} \\
o p \text { b }), \text { true }),(x, y) \mid x, y \in\{\&,|\wedge, \& \&,|||\} \wedge x \neq \\
y\}\end{array}$ \\
\hline ROR & Relational Operator Replacement & $\begin{array}{l}\{((\mathrm{a} o p \text { b }), \text { false }),((\text { a } o p \text { b }), \text { true }),(x, y) \mid x, y \in \\
\{>,>=,<,<=,==, !=\} \wedge x \neq y\}\end{array}$ \\
\hline UOI & Unary Operator Insertion & $\begin{array}{l}\{(\text { cond }, ! \text { cond }),(v,-v),(v, \sim v),(v,--v),(v, v--) \\
(v,++v),(v, v++)\}\end{array}$ \\
\hline
\end{tabular}

of mutant operators based on the grammar of the language. Then, based on empirical studies, researchers tend to select subsets of them in order to improve the applicability and scalability of the method. Of course both the definition of operators and mutant selection (selection of representative subsets) form the two sides of the same coin. Since all possible operators are enormous if not infinite, the definition of small sets of them can be viewed as a subset selection (among all possible definitions). Here, we refer to mutant reduction as the process of selecting subsets of operators over a given sets of them. We discuss studies that define mutant operators in Section 5.1.1 and mutant reduction in Section 5.1.2.

Based on the chosen set of mutant operators, we generate a set of mutant instances that we use to perform our analysis. Thus, our test objectives are to kill the mutants (design test cases that kill all the mutants). We can define as 'mutation score' or mutation coverage the ratio of mutants that are killed by our test cases. In essence, the mutation score denotes the degree of achievement of our test cases in fulfilling the test objectives. Thus, mutation score can be used as an adequacy metric [32]. 
Adequacy criteria are defined as predicates defining the objectives of testing [32. Goodenough and Gerhart [35] argue that criteria capture what properties of a program must be exercised to constitute a thorough test, i.e., one whose successful execution implies no errors in a tested program. Therefore, the use of mutation testing as a test criterion has the following three advantages [6]: to point out the elements that should be exercised when designing tests, to provide criteria for terminating testing (when coverage is attained), and to quantify test suite thoroughness (establish confidence).

In practice using mutation score as adequacy measure, implicitly assumes that all mutants are of equal value. Unfortunately, this is not true [36. In practice, some mutants are equivalent, i.e., they form functionally equivalent versions of the original program, while some others are redundant, i.e., they are not contributing to the test process as they are killed whenever other mutants are killed. Redundant mutants are of various forms. We have the duplicated mutants, mutants that are equivalent between them but not with the original program [37. We also have the subsumed mutants [38. (also called joint mutants [39]), i.e., mutants that are jointly killed when other mutants are killed [36, 39].

The problem with subsumed mutants is that they do not contribute to the test assessment process because they are killed when other mutants are also killed. This means that eliminating these mutants does not affect the selection/generation of test cases but the computation of the mutation score. Thus, the metric is inflated and becomes hard to interpret. As the distribution of mutants tend to be unpredictable and the identification of mutant equivalences and redundancies is an undecidable problem [37, it is hard to judge the test suite strengths based on the mutation score. In other words, the accuracy of the score metric is questionable. We will discuss this issue in depth in Section 9

\section{What is special about mutation testing}

Mutation testing principles and concepts share on a long heritage with more general scientific investigation, essentially drawing on the common sense of 'trial 
and error' (that pre-dates civilisation), and also resting on the foundations of inferential statistics and Popperian science [40].

One of the fundamental problems in software testing is the inability to know practically or theoretically when one has tested sufficiently. Practitioners often demand of researchers a method to determine when testing should cease. Unfortunately, this revolves around the question of what is intended by sufficiency; if we are to test in order to be sufficient to demonstrate the absence of bugs, then we are forced against the impossibility of exhaustive testing.

Faced with this challenge, much literature has centred on concepts of coverage, which measure the degree of test effort, that each coverage technique's advocates hope will be correlated with test achievement. For instance Table 2 reports on the main studies on this subject. There has been much empirical evidence concerning whether coverage is correlated with faults revelation [41 43], a problem that remains an important subject of study to the present day [6, 41]. However, even setting aside the concern of whether such correlation exists, nonmutation-based forms of coverage suffer from a more foundational problem; they are essentially unfalsifiable (with respect to the goal of fault revelation), in the Popperian sense of science 40.

Table 2: Summary of the main studies concerned with the relationship of test criteria and faults.

\begin{tabular}{|c|c|c|c|}
\hline $\begin{array}{l}\text { Author(s) [Refer- } \\
\text { ence }]\end{array}$ & Year & $\begin{array}{l}\text { Test Crite- } \\
\text { rion }\end{array}$ & $\begin{array}{l}\text { Summary of Primary Scientific } \\
\text { Findings }\end{array}$ \\
\hline Frankl \& Weiss 44 & '91, '93 & $\begin{array}{l}\text { branch, all- } \\
\text { uses }\end{array}$ & $\begin{array}{l}\text { All-uses relates with test effectiveness, } \\
\text { while branch does not. }\end{array}$ \\
\hline Offutt et al. 46. & '96 & $\begin{array}{l}\text { all-uses, } \\
\text { mutation }\end{array}$ & $\begin{array}{l}\text { Both all-uses and mutation are effective } \\
\text { but mutation reveals more faults. }\end{array}$ \\
\hline Frankl et al. 47 & '97 & $\begin{array}{l}\text { all-uses, } \\
\text { mutation }\end{array}$ & $\begin{array}{l}\text { Test effectiveness (for both all-uses and } \\
\text { mutation) is increasing at higher cover- } \\
\text { age levels. Mutation performs better. }\end{array}$ \\
\hline $\begin{array}{l}\text { Frankl } \quad \& \quad \text { Iak- } \\
\text { ounenko } 48\end{array}$ & '98 & $\begin{array}{l}\text { all-uses, } \\
\text { branch }\end{array}$ & $\begin{array}{l}\text { Test effectiveness increases rapidly at } \\
\text { higher levels of coverage (for both all-uses } \\
\text { and branch). Both criteria have similar } \\
\text { test effectiveness. }\end{array}$ \\
\hline Briand \& Pfahl 49 & '00 & $\begin{array}{l}\text { block, c- } \\
\text { uses, p-uses, } \\
\text { branch }\end{array}$ & $\begin{array}{l}\text { There is no relation (independent of test } \\
\text { suite size) between any of the four criteria } \\
\text { and effectiveness }\end{array}$ \\
\hline
\end{tabular}


Table 2: Summary of the main studies concerned with the relationship of test criteria and faults.

\begin{tabular}{|c|c|c|c|}
\hline $\begin{array}{l}\text { Author(s) [Refer- } \\
\text { ence }]\end{array}$ & Year & $\begin{array}{l}\text { Test Crite- } \\
\text { rion }\end{array}$ & $\begin{array}{l}\text { Summary of Primary Scientific } \\
\text { Findings }\end{array}$ \\
\hline Chen et al. 50 & 01 & Block & $\begin{array}{l}\text { coverage can be used for predicting the } \\
\text { software failures in operation. }\end{array}$ \\
\hline Andrews et al. 42 & '06 & $\begin{array}{l}\text { block, c- } \\
\text { uses, p-uses, } \\
\text { branch }\end{array}$ & $\begin{array}{l}\text { Block, c-uses, p-uses and branch coverage } \\
\text { criteria correlate with test effectiveness. }\end{array}$ \\
\hline Namin \& Andrews & '09 & $\begin{array}{l}\text { block, c- } \\
\text { uses, p-uses, } \\
\text { branch }\end{array}$ & $\begin{array}{l}\text { Both test suite size and coverage influ- } \\
\text { ence (independently) the test effective- } \\
\text { ness }\end{array}$ \\
\hline Li et al. 52 & '09 & $\begin{array}{l}\text { prime path, } \\
\text { branch, all- } \\
\text { uses, muta- } \\
\text { tion }\end{array}$ & $\begin{array}{l}\text { Mutation testing finds more faults than } \\
\text { prime path, branch and all-uses. }\end{array}$ \\
\hline $\begin{array}{l}\text { Papadakis } \quad \& \\
\text { Malevris 53 }\end{array}$ & '10 & $\begin{array}{l}\text { Mutant } \\
\text { sampling, } \\
1^{\text {st } \& ~ 2^{\text {nd }}} \\
\text { order muta- } \\
\text { tion }\end{array}$ & $\begin{array}{l}1^{\text {st }} \text { order mutation is more effective than } \\
2^{\text {nd }} \text { order and mutant sampling. There } \\
\text { are significantly less equivalent } 2 \text { nd order } \\
\text { mutants than } 1^{\text {st }} \text { order ones. }\end{array}$ \\
\hline Ciupa et al. 54 & '11 & $\begin{array}{l}\text { Random } \\
\text { testing }\end{array}$ & $\begin{array}{l}\text { Random testing is effective and has pre- } \\
\text { dictable performance. }\end{array}$ \\
\hline Kakarla et al. 55 & '11 & mutation & $\begin{array}{l}\text { Mutation-based experiments are vulner- } \\
\text { able to threats caused by the choice of } \\
\text { mutant operators, test suite size and pro- } \\
\text { gramming language. }\end{array}$ \\
\hline Wei et al. 56 & $' 12$ & Branch & $\begin{array}{l}\text { Branch coverage has a weak correlates } \\
\text { with test effectiveness. }\end{array}$ \\
\hline Baker \& Habli 57 & '13 & $\begin{array}{l}\text { statement, } \\
\text { branch, } \\
\text { MC/DC, } \\
\text { mutation, } \\
\text { code review }\end{array}$ & $\begin{array}{l}\text { Mutation testing helps improving the test } \\
\text { suites of two safety-critical systems by } \\
\text { identifying shortfalls where traditional } \\
\text { structural criteria and manual review } \\
\text { failed. }\end{array}$ \\
\hline Hassan \& Andrews & '13 & $\begin{array}{l}\text { multi-Point } \\
\text { Stride, data } \\
\text { flow, branch }\end{array}$ & $\begin{array}{l}\text { Def-uses is (strongly) correlated with test } \\
\text { effectiveness and has almost the same } \\
\text { prediction power as branch coverage. } \\
\text { Multi-Point Stride provides better pre- } \\
\text { diction of effectiveness than branch cov- } \\
\text { erage. }\end{array}$ \\
\hline $\begin{array}{l}\text { Gligoric et al. } 59 \\
60\end{array}$ & '13, '15 & $\begin{array}{l}\text { AIMP, } \\
\text { DBB, } \\
\text { branch, } \\
\text { IMP, PCC, } \\
\text { statement }\end{array}$ & $\begin{array}{l}\text { There is a correlation between coverage } \\
\text { and test effectiveness. Branch coverage is } \\
\text { the best measure for predicting the qual- } \\
\text { ity of test suites. }\end{array}$ \\
\hline
\end{tabular}


Table 2: Summary of the main studies concerned with the relationship of test criteria and faults.

\begin{tabular}{|c|c|c|c|}
\hline $\begin{array}{l}\text { Author(s) [Refer- } \\
\text { ence }]\end{array}$ & Year & $\begin{array}{l}\text { Test Crite- } \\
\text { rion }\end{array}$ & $\begin{array}{l}\text { Summary of Primary Scientific } \\
\text { Findings }\end{array}$ \\
\hline $\begin{array}{l}\text { Inozemtseva } \\
\text { Holmes } 61\end{array}$ & '14 & $\begin{array}{l}\text { statement, } \\
\text { branch, } \\
\text { modified } \\
\text { condition }\end{array}$ & $\begin{array}{l}\text { There is a correlation between coverage } \\
\text { and test effectiveness when ignoring the } \\
\text { influence of test suite size. This is low } \\
\text { when test size is controlled. }\end{array}$ \\
\hline Just et al. 43 & '14 & $\begin{array}{l}\text { statement, } \\
\text { mutation }\end{array}$ & $\begin{array}{l}\text { Both mutation and statement coverage } \\
\text { correlate with fault detection, with mu- } \\
\text { tants having higher correlation. }\end{array}$ \\
\hline Gopinath et al. 62 & '14 & $\begin{array}{l}\text { statement, } \\
\text { branch, } \\
\text { block, path }\end{array}$ & $\begin{array}{l}\text { There is a correlation between coverage } \\
\text { and test effectiveness. Statement cov- } \\
\text { erage predicts best the quality of test } \\
\text { suites. }\end{array}$ \\
\hline Ahmed et al. 63 & '16 & $\begin{array}{l}\text { statement, } \\
\text { mutation }\end{array}$ & $\begin{array}{l}\text { There is a weak correlation between cov- } \\
\text { erage and number of bug-fixes }\end{array}$ \\
\hline Ramler et al. 64 & $' 17$ & $\begin{array}{l}\text { strong mu- } \\
\text { tation }\end{array}$ & $\begin{array}{l}\text { Mutation testing provides valuable guid- } \\
\text { ance towards improving the test suites of } \\
\text { a safety-critical industrial software sys- } \\
\text { tem }\end{array}$ \\
\hline Chekam et al. 6] & $' 17$ & $\begin{array}{l}\text { statement, } \\
\text { branch, } \\
\text { weak \& } \\
\text { strong } \\
\text { mutation }\end{array}$ & $\begin{array}{l}\text { There is a strong connection between cov- } \\
\text { erage attainment and fault revelation for } \\
\text { strong mutation but weak for statement, } \\
\text { branch and weak mutation. Fault revela- } \\
\text { tion improves significantly at higher cov- } \\
\text { erage levels. }\end{array}$ \\
\hline $\begin{array}{l}\text { Papadakis et al. } \\
41]\end{array}$ & '18 & mutation & $\begin{array}{l}\text { Mutation score and test suite size corre- } \\
\text { late with fault detection rates, but often } \\
\text { the individual (and joint) correlations are } \\
\text { weak. Test suites of very high mutation } \\
\text { score levels enjoy significant benefits over } \\
\text { those with lower score levels. }\end{array}$ \\
\hline
\end{tabular}

Mutation testing is important because it provides a mechanism by which assertions concerning test effectiveness become falsifiable; failure to detect certain kinds of mutants suggest failure to detect certain kinds of faults. Alternative coverage criteria can only be falsified in the sense of stating that, should some desired coverage item remain uncovered, then claims to test effectiveness remain 'false'. Unfortunately, it is not usually possible to cover every desired coverage item, it is typically undecidable whether this criterion has been achieved in 
any case [59]. Coverage of all mutants is also undecidable 37], but mutation testing forms a direct link between faults and test achievements, allowing more scientific (in the sense intended by Popper) statements of test achievement than other less-fault-orientated approaches.

Mutation testing also draws on a rich seam of intellectual thought, that is currently becoming more popular in other aspects of science and engineering. Such counterfactual reasoning, can even be found beyond science, in the humanities, where historians use it to explore what would have happened, had certain historical events failed to occur. This is a useful intellectual tool to help increase understanding and analysis of the importance of these events the influence (or forward dependence [65] as we might think of it within the more familiar software engineering setting. In software engineering, counterfactual reasoning plays a role in causal impact analysis [66], allowing software engineers to discover the impact of a particular event, thereby partly obviating the problem 'correlation is not causation'.

In mutation testing, we create a 'counterfactual version of the program' (a mutant) that represents what the program would have looked like had it contained a specific chosen fault, thereby allowing us to investigate what would have happened if the test approach encounter a program containing such a fault. Causal impact analysis relies on recent development in statistics. In more traditional statistical analysis, mutation testing also finds a strong resonance with the foundations of sampling and inferential frequentist statistical analysis. A statistician might, for instance, seek to estimate the number of fish in a pool by catching a set of fish, marking these and returning them to the pool, subsequently checking how many marked fish are present in a random sample. Of course such an approach measures not only the number of fish in the pool, but also the effectiveness of the re-catching approach used in re-sampling. In a similar way, creating sets of mutant programs (marking fish) and then applying the test technique (re-sampling) can also be used to estimate the number of faults in the program, albeit confounded by concurrently measuring the effectiveness of the testing technique. 


\section{The relations between Mutants and Fault Revelation}

The underlying idea of mutation is simple; we form defects and we ask testers to design test cases that reveal them. Naturally, readers may ask why such an approach is effective. Literature answers this question in the following ways:

- First, (theoretically) by revealing the formed defects we can demonstrate that these specific types of defects are not present in the program under analysis [67. In such a case we assume that the formed mutants represent the fault types that we are interested in. In a broader perspective, the mutants used are the potential faults that testers target and thus, they are in a sense equivalent to real faults.

- Second, (theoretically and practically) when test cases reveal simple defects such as mutants (defects that are the result of simple syntactic alterations), they are actually powerful enough to reveal more complex defects. In such a case, we assume that test cases that reveal the used types of defects also reveal more complex types (multiple instances of the types we used) 68. Thus, mutation helps revealing a broader class of faults (than those used) composed of the simple and complex types of faults that were used.

- Third, (practically) when we design test cases that kill mutants we are actually writing powerful test cases. This is because we are checking whether the defects we are using can trigger a failure at every location (or related ones) we are applying them to. In such a case, we assume that test cases that are capable of revealing mutants are also capable of revealing other types of faults (different from the mutants). This is because mutants require checking whether test cases are capable of propagating corrupted program states to the observable program output (asserted by the test cases). Thus, potential faulty states (related to the mutants) have good chances to became observable [6]. 
The above mentioned points motivated researchers to study and set the foundation of mutation testing.

The first point has been shown theoretically based on the foundations of fault-based testing [67, 69. The practical importance of this assertion is that in case we form the employed mutants as common programming mistakes, then we can be confident that testers will find them. Thus, we can check against the most frequent faults. This premise becomes more important when we consider the Competent Programmer Hypothesis [24, which states that developers produce programs that are nearly correct, i.e., they require a few syntactic changes to reach the correct version. This hypothesis implies that if we form mutants by making few simple syntactic changes we can represent the class of frequent faults (made by "competent programmers"). A recent study by Gopinath et al. [70] has shown that defects mined from repositories involve three to four tokens to be fixed, confirming to some extent the hypothesis. Generally, the recent studies have not consider this subject and thus, further details about the competent programmer hypothesis can be found in the surveys of Offutt and Untch [26] and Jia and Harman [27].

The second point is also known as the mutant coupling effect [68. According to Offutt 68 the mutant coupling effect states "Complex mutants are coupled to simple mutants in such a way that a test data set that detects all simple mutants in a program will detect a large percentage of the complex mutants". Therefore, by designing test cases that reveal almost all the mutants used, we expect a much larger set of complex mutants to be also revealed. This premise has been studied both theoretically and practically (details can be found in the surveys of Offutt and Untch [26] and Jia and Harman [27]). Recent studies on the subject only investigated (empirically) the relationship between simple and complex mutants. For example the study of Gopinath et al. [71] demonstrates that many hihger order mutants are semantically different from the simple first order ones they are composed of. However, the studies of Langdon et al. 72 and Papadakis and Malevris 53. show that test cases kill a much larger ratio of complex (higher order) mutants than simple ones (first order ones) indicating 
that higher order mutants are of relatively lower strength than the first order ones.

The third point is a realisation of the requirement that the mutants must influence the observable output of the program (the test oracle). To understand the importance of this point we need to consider the so-called RIPR model (Reachability, Infection, Propagation Revealability) [32. The RIPR model states that in order to reveal a fault, test cases must: a) reach the faulty locations (Reachability), b) cause a corruption (infection) to the program state (Infection), c) propagate the corruption to the program output (Propagation) and d) cause a failure, i.e., make the corrupted state observable to the user, be asserted by the test cases (Revealability). Therefore, when designing test cases to kill mutants, we check the sensitivity of erroneous program states to be observable. Empirical evidence by Chekam et al. [6] has shown that this propagation requirement makes mutation strong and distinguishes it from weak mutation and other structural test criteria. In particular the same study demonstrates that mutant propagation is responsible for the revealation of $36 \%$ of the faults that can be captured by strong mutation.

Overall, the fundamental premise of mutation testing can be summarised as "if the software contains a fault, there will usually be a set of mutants that can only be killed by a test case that also detects that fault" [73]. This premise has been empirically investigated by the study of Chekam et al. [6] which demonstrated a strong connection between killing mutants and fault revelation. Similarly, the studies of Baker and Habli [57, Ramler et al. [64] and [74] have shown that mutation testing provides valuable guidance towards improving existing test suites.

A last reason that makes mutation strong is the fact that its test objectives are the formed defects. Thus, depending on the design of these defects, several test criteria can be emulated. Therefore, mutation testing has been found to be a strong criterion that subsumes, or probably subsumes $\{$ almost all other test

\footnotetext{
${ }^{1}$ Subsumption is not guaranteed but it is probable to happen 32 .
} 
criteria [32]. Thus, previous research has shown that strong mutation probably subsumes weak mutation [75], all dataflow criteria [47, 55], logical criteria [76], branch and statement criteria [52]. These studies suggest that a small set of mutant operators can often result in a set of test cases that is as strong as the ones resulting from other criteria.

\section{The Regular Code-based Mutation Testing Process}

This section details the code-based mutation testing advances. We categorise and present the surveyed advances according to the stages of the mutation testing process that they apply to. But before we begin the presentation we have to answer the following question: what is the mutation testing process? Figure 3 presents a detailed view of the modern mutation testing process. This process forms an extension of the one proposed by Offutt and Untch [26]. The extension is based on the latest advances in the area. The important point here is that the process keeps the steps that are inherently manual outside the main loop of the testing process (steps in bold). The remaining steps can be sufficiently automated (although manual analysis may still be necessary).

Overall, the process goes as follows: First, we select a set of mutants that we want to apply (Step 1, detailed in Section 5.1) and instantiate them by forming actual executable programs (Step 2, detailed in Section 5.2). Next, we need to remove some problematic mutants, such as equivalent, i.e., mutants that are semantically equivalent to the original program despite being syntactically different, and redundant mutants, i.e., mutants that are semantically different to the original program but are subsumed by others, (Step 3, detailed in Section 5.3 .

Once we form our set of mutants we can generate our mutation-based test suite, execute it against our mutants and determine a score. In this step, we design (either manually or automatically) test cases that have the potential to kill our mutants (Step 4, detailed in Section 5.4 and execute them with all the mutants (Step 5, detailed in Section 5.5) to determine how well they scored 


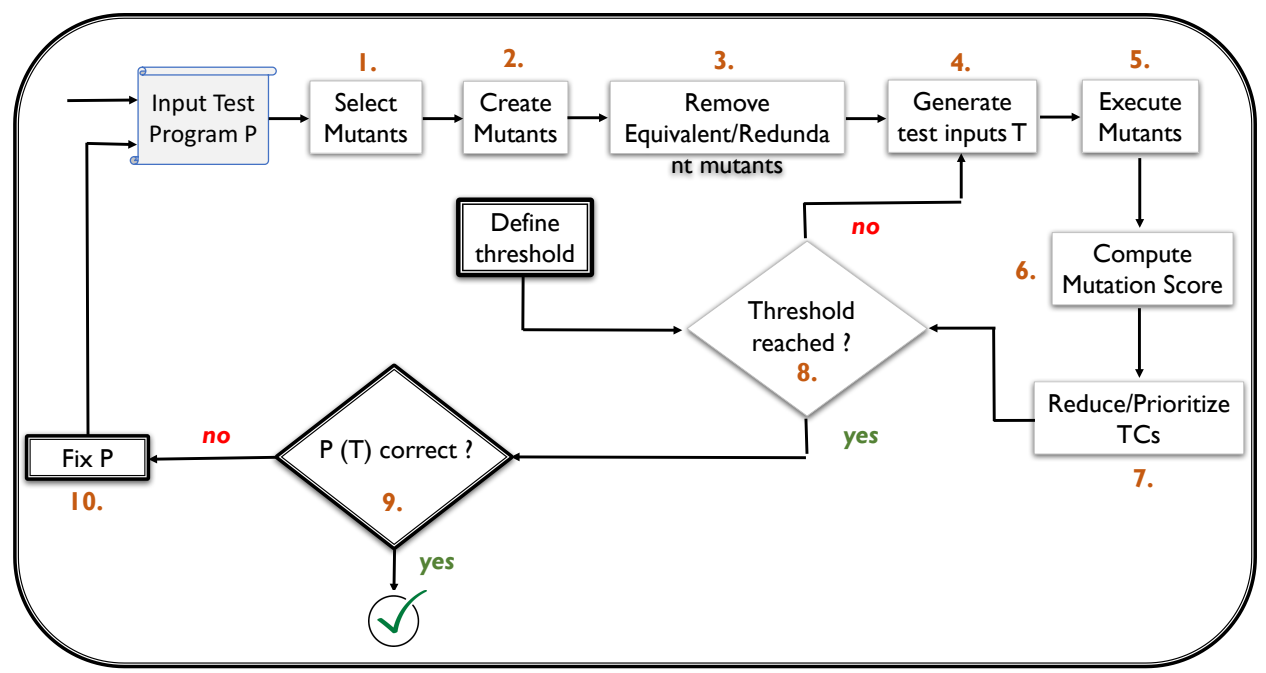

Figure 3: Modern Mutation Testing Process. The process forms an adaptation of the Offutt's and Untch's proposition [26] based on the latest advances in the area. Bold boxes represent steps where human intervention is mandatory.

(Step 6, detailed in Section 5.6). Subsequently, we perform test suite reduction by removing potentially ineffective test cases from our test suite. At this stage, we can also perform test case prioritisation so that we order the most effective test cases first (Step 7, detailed in Section 5.7). The steps 4 to 7 are repeated until the process results in a mutation score that is acceptable (Step 8, detailed in Section 5.8. .

The last part of the process is when the user is asked to assess whether the results of the test executions were the expected ones (Step 9, detailed in Section 5.9p. This step regards the so-called test oracle creation problem that involves the tester to assert the behaviour of the test execution. In case faults are found then developers need to fix the problems and relaunch the process (Step 10, detailed in Section 5.10 until we reach an acceptable score level and cannot find any faults. 


\subsection{Mutant Selection (Step 1.)}

Mutation testing requires selecting a set of mutant operators based on which the whole process is applied. Thus, we need to define the specific syntactic transformations (mutant operators) that introduce mutants. Since defining mutant operators requires the analysis of the targeted language these may result in an enormous number of mutants. Therefore, in practice it might be important to select representative subsets of them. The following subsection (named Mutant Operators) refers to works that define mutant operators, while the next one (named Mutant Reduction Strategies) refers to strategies that select subsets of mutants from a given set of mutants (aiming at reducing the cost of the process).

\subsubsection{Mutant Operators}

A large amount of work has focused on designing mutant operators that target different (categories of) programming languages, applications, types of defects, programming elements, and others.

\section{Operators for Specific Programming Languages.}

Anbalagan and Xie [7] proposed a mutant generation framework for AspectJ, an aspect-oriented programming language. This framework uses two mutant operators; pointcut strengthening and pointcut weakening, which are used to increase or reduce the number of joint points that a pointcut matches.

Derezinska and Kowalski [8] introduced six object-oriented mutant operators designed for the intermediate code that is derived from compiled $C \#$ programs. Their work also revealed that mutants on the intermediate language level are more efficient than the high-level source code level mutants.

Estero-Botaro et al. [78] defined 26 mutant operators for $W S-B P E L$ - the Web Services Business Process Execution Language. Later on, they further quantitatively evaluated these operators regarding the number of stillborn and equivalent mutants each operator generates [79]. On the same topic, BoubetaPuig et al. 80] conducted a quantitative comparison between the operators for $W S-B P E L$ and those for other languages. The results indicate that many of 
the WS-BPEL operators are different due to the lack of common features with other languages (e.g., functions and arrays).

Mirshokraie et al. [10, 81] proposed a set of JavaScript operators. These are designed to capture common mistakes in JavaScript (such as changing the setTimeout function, removing the this keyword, and replacing undefined with null). Experimental results indicate the efficiency of these operators in generating non-equivalent mutants.

Delgado-Pérez [7, 82] conducted an evaluation of class-level mutant operators for $\mathrm{C}++$. Based on the results, they propose a $\mathrm{C}++$ mutation tool, $\mathrm{MuCPP}$, which generates mutants by traversing the abstract syntax tree of each translation unit with the Clang API.

\section{Operators for Specific Categories of Programming Languages.}

Derezinska and Kowalski [8] explored and designed the mutant operators for object-oriented programs through $C \#$ programs. They advocated that traditional mutant operators are not sufficient for revealing object-oriented flaws. Hu et al. 83 studied in depth the equivalent mutants generated by object-oriented class-level mutant operators and revealed differences between class-level and statement-level mutation: statement-level mutants are more easy to be killed by test cases.

Ferrari et al. 84 focused on aspect-oriented programs. They design a set of operators based on the aspect-oriented fault types. Similarly to the work of Anbalagan and Xie [77, this work uses Aspect J as a representative of aspectoriented programs. Except for pointcut-related operators, operators for general declarations, advice definitions and implementations are also adopted.

Bottaci [85] introduced an mutation analysis approach for dynamically-typed languages based on the theory that the mutants generated by modifying types are very easily killed and should be avoided. Gligoric et al. [86] mentioned that "almost all mutation tools have been developed for statically typed languages", and thus proposed SMutant, a mutation tool that postpones mutation until execution and applies mutation testing dynamically instead of statically. In this way, the tool is able to capture type information of dynamic languages 
during execution.

\section{Operators for Specific Categories of Applications.}

Alberto et al. 87] investigated the mutation testing approach for formal models. In particular, they introduced an approach to apply mutation testing to Circus specifications as well as an extensive study of mutant operators. Praphamontripong et al. [88, 89] and Mirshokraie et al. [10] designed and studied mutant operators for web applications. Example operators are link/field/transition replacement and deletion. In a follow up study, Praphamontripong and Offutt [90] refined the initial set of operators (they exclude 3 operators), i.e., operators proposed in [89, by considering the redundancy among the mutants they introduce.

Deng et al. [91, 92 defined mutant operators specific for the characteristics of Android apps, such as the event handle and the activity lifecycle mutant operators. Usaola et al. [93] introduced an abstract specification for defining and implementing operators for context-aware, mobile applications. Similarly, Linares-Vasquez et al. 94] introduced 38 mutation operators for Android apps. These operators were systematically derived by manually analysis types of Android faults. Oliveira et al. 95] proposed 18 GUI-level mutant operators. Also focused on GUI mutants, Lelli et al. 96] specially designed mutation operators based on the created fault model. Abraham and Erwig [97] proposed operators for spreadsheets. Dan and Hierons 98, introduced how to generate mutants aiming at floating-point comparison problems. Jagannath et al. 99] introduced how to generate mutants for actor systems.

Maezawa et al. 100] proposed a mutation-based method for validating Asynchronous JavaScript and XML (Ajax) applications. The approach is based on delay-inducing mutant operators that attempt to uncover potential delaydependent faults. The experimental study suggests that by killing these mutants, actual errors can be revealed.

The study of Xie et al. 101] describes a mutation-based approach to analyse and improve Parameterized Unit Tests (PUTs). The authors propose appropriate mutant operators that alter the effectiveness of the PUT test by varying the 
strength of its assumptions and assertions.

\section{Operators for Specific Categories of Bugs.}

Brown et al. $\quad 102$ proposed a technique to mine mutation operators from source code repositories. The intuition of this work is that by making mutants sintactically similar to real faults one can get semantically similar mutants. Loise et al. [19] uses mutation testing to tackle security issues. They proposed 15 security-aware mutant operators for Java. Nanavati et al. [103, 104] realised that few operators are able to simulate memory faults. They proposed 9 memory mutant operators targeting common memory faults. Garvin and Cohen [105] focus on feature interaction faults. An exploratory study was conducted on the real faults from two open-source projects and mutants are proposed to mimic interaction faults based on the study's results. Al-Hajjaji et al. [106] specially focus on variability-based faults, such as feature interaction faults, feature independency faults, and insufficient faults. Based on real variability-related faults, they derive a set of mutant operators for simulating them.

Additionally, other studies focus on the design of mutant operators for different levels or different programming elements. Mateo et al. [5] defined systemlevel mutant operators. Applying mutation at the system level faces two problems: first, mutating one part of the system can lead to an anomalous state in another part, thus comparing program behaviour is not a trivial task; and, second, mutation's execution cost. To resolve these problems, the authors turn to weak mutation by introducing flexible weak mutation for the system level. The proposed approach is embedded in a mutation tool named Bacterio. Delamaro et al. 107] designed three new deleting mutant operators that delete variables, operators, and constants.

Additional categories of operators are due to Belli et al. [108, who proposed mutant operators for go-back functions (which cancel recent user actions or system operations), including basic mutant operators (i.e., transaction, state, and marking mutant operators), stack mutant operators (i.e., write replacement and read replacement), and high order mutant operators.

Gopinath and Walkingshaw 109 proposed operators targeting type anno- 
tations. This line of work aims at evaluating the appropriateness of type annotations. Jabbarvand and Malek [110] introduced an energy-aware framework for Android application. In this work, a set of fifty mutant operators mimicing energy defects was introduced. Arcaini et al. 111] proposed operators targeting regular expressions. These aim at assisting the generation of tests based on a fault model involving the potential mistakes one could made with regex.

\subsubsection{Mutant Reduction Strategies}

Mutant reduction strategies aim at selecting representative subsets from given sets of mutants. The practical reason for that is simply to reduce the application cost of mutation (since all the costly parts depend on the number of mutants).

Perhaps the simpler way of reducing the number of mutants is to randomly pick them. This approach can be surprisingly effective and achieve reasonably good trade-offs. Papadakis and Malevris 53] report that randomly selecting $10 \%, 20 \%, 30 \%, 40 \%, 50 \%$ and $60 \%$ of the mutants results in a fault loss of approximately $26 \%, 16 \%, 13 \%, 10 \%, 7 \%$ and $6 \%$ respectively. Zhang et al. 112 reports that by killing randomly selected sets of mutants, composed of more than $50 \%$ of the initial set, results in killing more than $99 \%$ of all the mutants. Recently, Gopinath et al. [113] used large open source programs and found that a small constant number of randomly selected mutants is capable of providing statistically similar results to those obtained when using all mutants. Also, they found that this sample is independent of the program size and the similarity between mutants.

An alternative way of selecting mutants is based on their types. The underlying idea is that certain types of mutants may be more important than others and may result in more representative subsets than random sampling. Namin et al. 114 used a statistical analysis procedure to identify a small set of operators that sufficiently predicts the mutation score with high accuracy. Their results showed that it is possible to reduce the number of mutants by approximately $93 \%$. This is potentially better than the mutant set of the pre- 
vious studies, i.e., the 5-operator set of Offutt et al. 34. Investigating ways to discover relatively good trade-offs between cost and effectiveness, Delgado et al. 82 studied a selective approach that significantly reduce the number of mutants with a minimum loss of effectiveness for $\mathrm{C}++$ programs. Delamaro et al. [107, 115] experimented with mutants that involve deletion operations (delete statements or part of it) and found that they form a cost-effective alternative to other operators (and selective mutation strategies) as it was found that they produce significantly less equivalent mutants. In a later study, Durelli et al. [116] studied whether the manual analysis involved in the identification of deletion equivalent mutants differs from that of other mutants and found no significant differences. The same study also reports that relational operators require more analysis in order to asses their equivalence.

A later study of Yao et al. [117] analysed the mutants produced by the 5-operator set of Offutt et al. 34 and found that equivalent and stubborn mutants are highly unevenly distributed, Thus, they proposed dropping the ABS class and a subclass of the UOI operators (post increment and decrement) to reduce the number of equivalent mutants and to improve the accuracy of the mutation score. Zhang et al. [118, 119] conducted an empirical study regarding the scalability of selective mutation and found that it scales well for programs involving up to 16,000 lines of code. To further improve scalability, Zhang et al. 120] demonstrated that the use of random mutant selection with $5 \%$ of the mutants (among the selective mutant operators) is sufficient for predicting the mutation score (of the selective mutants) with high accuracy.

A comparison between random mutant selection and selective mutation was performed by Zhang et al. [112. In this study, it was found that there are no significant differences between the two approaches. Later, Gopinath et al. [121] reached a similar conclusion by performing a theoretical and empirical analysis of the two approaches. The same study also concludes that the maximum possible improvement over random sampling is $13 \%$.

Overall, as we discuss in Section 9, all these studies were based on the traditional mutation scores (using all mutants) that is vulnerable to the "subsumed 
mutant threat" [36]. This issue motivated the study of Kurtz et al. [122, which found that mutant reduction approaches (selective mutation and random sampling) perform poorly when evaluated against subsuming mutants.

Both random sampling and selective mutation are common strategies in the literature. Gligoric et al. [123] applied selective mutation to concurrent programs and Kaminski and Ammann [124 to logic expressions. Papadakis and Traon [17, 125] adapts both of them for the context of fault localisation and reports that both random sampling and selective mutation that use more than $20 \%$ of all the mutants are capable of achieving almost the same results with the whole set of mutants.

Another line of research aiming at reducing the number of mutants is based on the notion of higher order mutants. In this case, mutants are composed by combining two or more mutants at the same time. Polo et al. [126] analysed three strategies to combine mutants and found that they can achieve significant cost reductions without any effectiveness loss. Later, studies showed that relatively good trade-offs between cost and effectiveness can be achieved by forming higher order combination strategies [39, 53, 127. In particular, Papadakis and Malevris [53] found that second order strategies can achieve a reduction of $80 \%$ to $90 \%$ of the equivalent mutants, with approximately $10 \%$ or less of test effectiveness loss. Similar results are reported in the studies of Kintis et al. [39], Madeyski et al. 28] and Mateo et al. [128, who found that second order strategies are significantly more efficient than the first order ones. Taking advantage of these benefits and ameliorate test effectiveness losses, Parsai et al. [129] built a prediction model that estimates the first-order mutation score given the achieved higher order mutation score.

Other attempts to perform mutant reduction are based on the mutants' location. Just et al. [130] used the location of the mutants on the program abstract syntax tree to model and predict the utility of mutants. Sun et al. 131 explored the program path space and selected mutants that are as diverse as possible with respect to the paths covering them. Gong et al. [132] selected mutants that structurally dominate the others (covering them results in cover- 
ing all the others). This work aims at weak mutation and attempts to statically identify dominance relations between the mutants. Similarly, Iida and Takada 133 identify conditional expressions that describe the mutant killing conditions, which are used for identifying some redundant mutants. Pattrick et al. 134] proposed an approach that identifies hard-to-kill mutants using symbolic execution. The underlying idea here is that mutants with little effect on the output are harder to kill. To determine the effect of the mutants on the program output, Pattrick et al. suggests calculating the range of values (on the numerical output expressions) that differ when mutants are killed. This method was latter refined by Pattrick et al. [135] by considering the semantics (in addition to numeric ones) of Boolean variables, strings and composite objects.

Another attempt to reduce the number of mutants is to rank them according to their importance. After doing so, testers can analyse only the number of mutants they can handle based on the available time and budget by starting from the higher ranked ones. The idea is that this way, testers will customise their analysis using the most important mutants. In view of this, Sridharan et al. [136] used a Bayesian approach that prioritises the selection of mutant operators that are more informative (based on the set of the already analysed mutants). Along the same lines, Namin et al. 137] introduced MuRanker an approach that predicts the difficulty and complexity of the mutants. This prediction is based on a distance function that combines three elements; the differences that mutants introduce on the control-flow-graph representation (Hamming distance between the graphs), on the Jimple representation (Hamming distance between the Jimple codes) and on the code coverage differences produced by a given set of test cases (Hamming distance between the traces of the programs). All these together allow testers to prioritise towards the most difficult to kill mutants.

Mirshokraie et al. [10, 81] used static and dynamic analysis to identify the program parts that are likely to either be faulty or to influence the program output. Execution traces are used in order to identify the functions that play an important role on the application behaviour. Among those, the proposed approach then mutates selectively: a) variables that have a significant impact 
on the function's outcome and b) the branch statements that are complex. The variables with significant impact on the outcome of the functions are identified using the usage frequency and dynamic invariants (extracted from the execution traces), while complex statements are identified using cyclomatic complexity.

Anbalagan and Xie [77] proposed reducing mutants, in the context of pointcut testing of AspectJ programs, by measuring the lexical distance between the original and the mutated pointcuts (represented as strings). Their results showed that this approach is effective in producing pointcuts that are both of appropriate strength and similar to those of the original program.

Finally, mutant reduction based on historical data has also been attempted. Nam et al. 138] generated calibrated mutants, i.e., mutants that are similar to the past defects of a project (using the project's fix patterns), and compared them with randomly selected ones. Their results showed that randomly selected mutants perform similarly to the calibrated ones. Inozemtseva et al. [139] proposed reducing the number of mutants by mutating the code files that contained many faults in the past.

\subsection{Mutant Creation (Step 2.)}

This stage involves the instantiation of the selected mutants as actual executables. The easiest way to implement this stage is to form a separate source file for each considered mutant. This approach imposes high cost as it requires approximately 3 seconds (on average) to compile a single mutant of a large project [37]. Therefore, researchers have suggested several techniques to tackle this problem.

The most commonly used technique realises the idea of meta-mutation, also known as mutant schemata [140, which encodes all mutants in a single file. This is achieved by parameterising the execution of the mutants [141]. The original proposition of mutant schemata involved the replacement of every pair of operands that participate in an operation with a call to a meta-function that functions as the operand [140]. The meta-functions are controlled through global parameters. This technique has been adopted by several tools and researchers, 
Papadakis and Malevris [141] use special meta-functions to monitor the mutant execution and control the mutant application. Wang et al. [142] and Tokumoto et al. [143] use meta-functions that fork new processes. Bardin et al. [144] and Marcozzi et al. [145, 146] instrument the program with meta-functions that do not alter the program state, called labels, to record the result of mutant execution at the point of mutation and apply weak mutation.

Another approach involves bytecode manipulation [9, 147. Instead of compiling the mutants, these approaches aim at generating mutants by manipulating directly the bytecode. Coles et al. adopt such an approach for mutating Java bytecode [147. Derezinska and Kowalski [8] and Hariri et al. [148] adopt the same approach for mutating the Common Intermediate Language of .NET and LLVM Bitcode, respectively.

Other approaches involve the use of interpreted systems, such as the ones used by symbolic evaluation engines [149. A possible realisation of this attempt is to harness the Java virtual machines in order to control and introduce the mutants [150]. Finally, Devroey et al. 151] suggested encoding all mutants as a product line. The mutants can then be introduced as features of the system under test.

\subsection{Statically Eliminating Equivalent and Redundant mutants (Step 3.)}

This step involves the identification of problematic mutants before their execution. This is a process that is typically performed statically. The idea is that some equivalent mutants, i.e., mutants that are semantically equivalent to the original program despite being syntactically different, and some redundant mutants, i.e., mutants that are semantically different to the original program but are subsumed by others, can be identified and removed prior to the costly test execution phase. By removing these "useless" types of mutants we gain two important benefits: first, we reduce the effort required to perform mutation and, second, we improve the accuracy of the mutation score measurement. Unfortunately, having too many "useless" mutants obscures the mutation testing score measurement by either overestimating or underestimating the level of coverage 
achieved. This last point is particularly important as it is linked to the decision of when to stop the testing process, i.e., Step 8 (Section 5.8).

\subsubsection{Identifying Equivalent Mutants}

Detecting equivalent mutants is a well-known undecidable problem [28]. This means that it is unrealistic to form an automated technique that will identify all the equivalent mutants. The best we can do is to form heuristics that can remove most of these mutants. One such effective heuristic relies on compiler optimisation techniques [37, 152]. The idea is that code optimisations transform the syntactically different versions (mutants) to the optimised version. Therefore, semantically equivalent mutants are transformed to the same optimised version. This approach is called Trivial Compiler Optimization (TCE) and works by declaring equivalences only for the mutants that their compiled object code is identical to the compiled object code of the original program. Empirical results suggest TCE is surprisingly effective, being able to identify at least $30 \%$ of all the equivalent mutants.

Other techniques that aim at identifying equivalent mutants are of Kintis and Malevris 153 155] who observed that equivalent mutants have specific dataflow patterns which form data-flow anomalies. Thus, by using static data-flow analysis we can eliminate a large portion of equivalent mutants. This category of techniques include the use of program verification techniques, such as Value Analysis and Weakest Precondition calculus. Program verification is used to detect mutants that are unreachable or mutants that cannot be infected [144, 145.

A different attempt to solve the same problem is based on identifying killable mutants. This has been attempted using (static) symbolic execution [149, 156. Such attempts aim at executing mutants symbolically in order to identify whether these can be killable with symbolic input data. Other approaches leverage software clones to tackle this issue [157. Since software clones behave similarly, their (non-)equivalent mutants tend to be the same. Therefore, likely killable mutants can be identified by projecting the mutants of one 
clone to the other [157.

Literature includes additional techniques for the identification of equivalent mutants using dynamic analysis. These require test case execution and, thus, are detailed in the Step 6 (Section 5.6.

\subsubsection{Identifying Redundant Mutants}

Redundant mutants, i.e., mutants that are killed when other mutants are killed, inflate the mutation score with the unfortunate result of skewing the measurement. Thus, it is likely that testers will not be able to interpret the score well and end up wasting resources or performing testing of lower quality than intended [152]. To this end, several researchers have proposed ways to statically reduce redundancies.

Researchers have identified redundancies between the mutants produced by the mutant operators. The initial attempts can be found in the studies of Foster [158, Howden [159] and Tai [160, 161] which claimed that every relational expression should only be tested to satisfy the $>,==$ and $<$ conditions. Tai 160 , 161] also argued that compound predicates involving $n$ conditional AND/OR operators should be tested with $n+2(2 * n+3)$ conditions. Along the same lines, Papadakis and Malevris [149, 162] suggested inferring the mutant infection conditions (using symbolic execution) of the mutants produced by all operators and simplify them in order to reduce the effort required to generate mutationbased test cases. This resulted in restricted versions for the Logical, Relational and Unary operators.

More recently, Kaminski et al. [76, 163] analysed the fault hierarchy of the mutants produced by the relational operators and showed that only three instances are necessary. Just et al. [164] used a similar analysis and identified some redundancies between the mutants produced by the logical mutant operators. In a subsequent work, Just et al. [165] showed that the unary operator can be also improved. Putting all these three cases together, i.e., Logical, Relational and Unary operators, results in significant gains in both required runtime execution (runtime reductions of approximately 20\%) and mutation score accuracy 
(avoiding mutation score overestimation which can be as high as 10\%). Along the same lines, Fernandes et al. [166] proposed 37 rules that can help avoiding the introduction of redundant mutants. Their results showed that these rules can reduce (on average) $13 \%$ of the total number of mutants.

All these approaches are based on a "local" form analysis, which is at the predicate level (designed for the weak mutation). Thus, applying them on strong mutation may not hold due to: a) error propagation that might prohibit killing the selected mutants [141 and b) multiple executions of the mutated statements caused by programming constructs such as loops and recursion [167, 168]. Empirical evidence by Lindström and Márki [168] confirms the above problem and shows that there is a potential loss on the mutation score precision of $8 \%$, at most.

Recently, Trivial Compiler Optimization (TCE) [37, 152] has been suggested as a way to reduce the adverse effects of this problem. Similar to the equivalent mutant identification, TCE identifies duplicate mutant instances by comparing the compiled object code of the mutants. Empirical results have shown that TCE identifies (on average) $21 \%$ and $5.4 \%$ of $\mathrm{C}$ and Java mutants 152 . The benefits of using TCE is that it is conservative as all declared redundancies are guaranteed and deals with strong mutation.

Finally, Kurtz et al. [169] attempts to identify non-redundant mutants using (static) symbolic execution. The idea is that by performing differential symbolic execution between the mutants it is possible to identify such redundancies.

\subsection{Mutation-based Test Generation (Step 4.)}

According to the RIPR model [32, in order to kill a mutant we need test cases that reach the mutant, cause an infection on the program state, manifest the infection to the program output at an observable to the user point (asserted by the test cases). Formulating these conditions as requirements we can drive the test generation process. Currently, there are three main families of approaches aiming at tackling this problem named as (static) constraint-based test generation [149, 170, search-based test generation [171, 172] and Concolic/Dynamic 
symbolic execution [141, 172]. Additional details regarding the automatic test generation and mutation-based test generation can be found in the surveys of Anand et al. [173] and Souza et al. [29].

\subsubsection{Static Constraint-based Test Generation}

Constraint-based methods turn each one of the RIPR conditions into a constraint and build a constraint system that is passed to a constraint solver. Thus, the mutant killing problem is converted to a constraint satisfaction problem [170. Wotawa et al. [174] and Nica [175] proposed formulating the original and mutant programs (one pair at a time) as a constraint system and use solvers to search for a solution that makes the two programs differ by at least one output value. Kurtz et al. [169] adopted the same strategy in order to identify subsuming mutants.

Papadakis and Malevris [149, 176] suggested formulating the RIPR conditions under selected paths in order to simplify the constraint formulation and resolution process. A usual problem of path selection is the infeasible path problem, i.e., paths that do not represent valid execution paths, which is heuristically alleviated using an efficient path selection strategy [149, 176].

Other attempts are due to Papadakis and Malevris [177] and Holling et al. 156] who used out of the box symbolic execution engines (JPF-SE [178] and KLEE [179] respectively) to generate mutation-based test cases. These approaches instrument the original program with mutant killing conditions that the symbolic execution engine is asked to cover (transforms the mutant killing problem to code reachability problem). Riener et al. [180] suggested using Bounded Model Checking techniques to search for solutions (counter examples) that expose the studied mutants.

\subsubsection{Concolic/Dynamic Symbolic Execution Test Generation}

To overcome the potential weaknesses of the static methods, researchers proposed dynamic techniques such as Concolic/Dynamic symbolic execution. Similar to the static methods, the objective is to formulate the RIPR conditions. 
However, dynamic techniques approximate the symbolic constraints based on the actual program execution. Therefore, there is a need to embed the mutant killing conditions within the executable program and guide test generation towards these conditions.

The first approach that uses Concolic/Dynamic symbolic execution is that of Papadakis et al. [177, 181] that targets weak mutation. The main idea of this approach is to embed the mutant infection conditions within the schematic functions that are produced by the mutant schemata technique (described earlier in Section 5.2. This way, all the mutants are encoded into one executable program along with their killing conditions (mutant infection conditions). Subsequently, by using a Concolic/Dynamic symbolic execution tool we can directly produce test cases by targeting the mutant infection conditions. Similarly, Zhang et al. [182] and Bardin et al. 144, 183] use annotations to embed the mutant infection conditions within the program under analysis. Along the same lines, Jamrozik et al. 184 augment the path conditions with additional constraints, similar to mutant infection conditions, to target mutants.

All the above approaches are actually performing some form of weak mutation as they produce test cases by targeting mutants' reachability and infection conditions. Performing weak mutation often results in tests that can strongly kill many mutants [32]. However, these tests often only kill (strongly) trivial mutants which usually fail to reveal faults [6]. To improve test generation, there is a need to formulate the mutant propagation condition on top of the reachability and infection conditions. This is complex as it involves the formulation of the two executions (the one of the original program and the one of the mutant) along all possible execution paths. Therefore, researchers try to heuristically approximate this condition through search. Papadakis and Malevris [141] search the path space between the mutation point until the program output. This helps finding inputs that satisfy the propagation condition. Finally, another approach proposed by Harman et al. [172] searches the program input space using a constrained search engine (reachability and infection conditions are augmented with an extra conjunct to additional constraints). 


\subsubsection{Search-based Test Generation}

Other dynamic test generation techniques use search-based optimisation algorithms to generate mutant killing test cases. The idea realised by this class of methods is to formulate and search the program input domain under the guidance of a fitness function. The primary concern for these approaches is to define a fitness function that is capable of capturing the RIPR conditions and effectively identify test inputs that satisfy these conditions.

There are many different search-based optimisation algorithms to choose from, but in the case of mutation, the most commonly used ones are the hill climbing [172, 185] and Genetic algorithms. As mentioned earlier, the main concern of these methods is the formulation of the fitness function. For instance, Ayari et al. [186] formulates the fitness as mutant reachability (distance from covering mutants) and Papadakis et al. [177, 181, formulates the fitness as fulfilment of mutant infection conditions (distance from infecting mutants).

As with the already-presented techniques, formulating the propagation condition in the fitness function is not straight-forward and thus, it is approximated by formulating indirect objectives. Fraser and Zeller [171, 187] measure the mutants' impact (the number of statements with changed coverage, between a mutant and the original programs, along the test execution) to form the propagation condition. Papadakis and Malevris [188 190 measure the distance to reach specific program points which when impacted (covered by the original program execution and not by the mutant execution or vice versa) result in mutant killing. These are determined based on the mutants that have been killed by the past executions.

Patrick et al. 191 proposed a technique to evolve input parameter subdomains based on their effectiveness in killing mutants. The experimental evaluation of this approach suggests that it can find optimised subdomains whose test cases are capable of killing more mutants than test cases selected from random subdomains.

The most recent approaches try to formulate the mutant propagation con- 
dition by measuring the disagreement between the test traces of the original program and the mutants. Fraser and Arcuri [192] count the number of executed predicates that differ while Souza et al. [185. measure the differences in the branch distances between the test executions.

\subsection{Mutant Execution (Step 5.)}

Perhaps the most expensive stage of mutation testing is the mutant execution step. This step involves the execution of test cases with the candidate test cases. Thus, given a program with $n$ mutants and a test suite that contains $m$ tests, we have to perform $n \times m$ program executions at maximum. For instance, consider a case where we have 100 mutants and a test suite that requires 10 seconds to execute for the original program. In this case, we expect that our analysis will complete in 1,000 seconds. This makes the process time-consuming (since a large number of mutants is typically involved) and, thus, limits the scalability of the method.

To reduce this overhead, several optimisations have been proposed. We identify two main scenarios where the optimisations may appear. The first one, which we refer to as "Scenario A", regards the computation of mutation score, while the second one, which we refer to as "Scenario B", regards the computation of a mutant matrix (a matrix that involves the test execution results of all tests with all the mutants; an example appears in Figure 4). This mutant matrix is used by many techniques such as the mutation-based fault localisation [17, the oracle construction [171, test suite reduction [193] and prioritisation [194].

The difference between the abovementioned scenarios is that when computing the mutation score (Scenario A) we only need to execute a mutant until it is killed. Therefore, we do not need to re-execute the mutants that have already been killed by a test case with other test cases. This simple approach achieves major execution savings. However, it does not apply on the second scenario where we need to execute all mutants with all test cases.

To illustrate the difference, consider the example mutant matrix of Figure 4 To construct this mutant matrix (Scenario B), we need 20 executions (4 mutants 


\begin{tabular}{lllll}
\hline & \multicolumn{4}{c}{ Mutants } \\
Tests & $m_{1}$ & $m_{2}$ & $m_{3}$ & $m_{4}$ \\
\hline$t_{1}$ & $\checkmark$ & & & $\checkmark$ \\
$t_{2}$ & $\checkmark$ & & & $\checkmark$ \\
$t_{3}$ & & & $\checkmark$ & $\checkmark$ \\
$t_{4}$ & & & $\checkmark$ & \\
$t_{5}$ & & & $\checkmark$ & $\checkmark$ \\
\hline
\end{tabular}

Figure 4: Example Mutant Matrix.

executed with the 5 tests). A naive approach for computing the mutation score (Scenario A) that does the same will also require 20 executions. However, mutation score calculation requires computing the number of mutants that are killed. Therefore, once a mutant is killed we do not need to re-execute it. In the above example, the tester will make 4 executions for $t_{1}$ (mutants $m_{1}, m_{2}$, $m_{3}$ and $\left.m_{4}\right)$ and he will determine that $m_{1}$ and $m_{4}$ are killed. Then, he will execute all the live mutants with $t_{2}\left(2\right.$ executions, mutants $m_{2}$ and $\left.m_{3}\right)$ and he will determine that none of them is killed. Then, he will execute the same mutants with $t_{3}$ (2 executions, mutants $m_{2}$ and $m_{3}$ ) and will determine that $m_{3}$ is killed. For the last couple of test cases $t_{4}$ and $t_{5}$ he will execute only the mutant $m_{2}$. The sum of these executions is 10 which is greatly reduced compared to the initial requirement of 20 executions.

In the above analysis, we implicitly consider that there is an order of the test cases we are using. Therefore, by using different orders we can reduce further the number of test executions. In the example of Figure 4 if we execute $t_{1}$ and $t_{3}$ first and then $t_{2}, t_{4}$ and $t_{5}$, we can reduce the number of test executions to 9. Zhang et al. 195] realised this idea using test case prioritisation techniques. Similarly, Just et al. [196] proposed using the fastest test cases first and Zhu et al. 197. selected pairs of mutants and test cases to run together based on the similarity of mutants and test cases (identified by data-compression techniques). Of course, these two approaches only apply to Scenario A. 
Regarding both Scenarios A and B, there are several optimisations that try to avoid executing mutants that have no chance of being killed by the candidate test cases. Thus, mutants that are not reachable by any test should not be executed as there is no chance of killing them. This is one of the initial test execution optimisations that has been adopted in the Proteum mutation testing tool [198. This tool records the execution trace of the original program and executes only the mutants that are reachable by the employed tests. In practice, this optimisation achieves major speed-ups compared to the execution of all tests (in both considered scenarios).

Papadakis and Malevris [177, 181] observed that it is possible to record with one execution all the mutants that can be infected by a test. This was implemented by embedding the mutant infection conditions within the schematic functions that are produced by the mutant schemata technique (described in Section 5.2. Thus, instead of executing every mutant with every test, it is possible to execute all mutants at once, by monitoring the coverage of the infection conditions. Durelli et al. [150] suggested harnessing the Java virtual machine instead of schematic functions in order to record the mutant infection conditions. Both these methods resulted in major speed-ups (up to 5 times).

When performing strong mutation many mutants are not killed despite being covered by test cases, simply because the mutant execution did not infect the program state. Therefore, there is no reason to strongly execute mutants that are not reached and infected by the candidate test cases. Based on this observation, Papadakis and Malevris [141] proposed to strongly execute only the mutants that are reached and infect the program state at the mutant expression point. Along the same lines, Kim et al. 199. proposed optimising test executions by avoiding redundant executions identified using statement level weak mutation. Both the studies of Papadakis and Malevris [141] and Kim et al. 199] resulted in major execution savings. More recently, Just et al. 200] reported that these approaches reduce the mutant execution time by $40 \%$. A further extension of this approach is to consider mutant infection at the mutant statement point (instead of mutant expression) [142]. 
Other test execution advances include heuristics related to the identification of infinite loops caused by mutants. Such infinite loops are frequent and greatly affect mutant execution time. However, since determining whether a test execution can terminate or not is an undecidable problem heuristic solutions are needed. The most frequent practice adopted by mutation testing tools to terminate test execution is with predefined execution time thresholds, e.g., if it exceeds three times the original program execution time. Mateo et al. [201, 202 proposed recording program execution and determine whether potential infinite loops are encountered by measuring the number of encountered iterations.

All the abovemetioned works aim at removing redundant executions. Another way to reduce the required effort is to take advantage of common execution parts (between the original program and the mutants). Thus, instead of executing every mutant from the input point to the mutation point, we can have a shared execution for these parts and then a different execution for the rest (from the mutation point to the program output). Such an approach is known as Split-stream execution [203]. The separation of the execution is usually performed using a Fork mechanism [143]. Empirical results suggest that such an approach can substantially improve the mutant execution process [142, 143]. It is noted that these approaches are orthogonal to those that are based on mutant infection. Therefore, a combination of them can further improve the process and substantially enhance the scalability of the method, as demonstrated by Wang et al. [142].

An alternative way of speeding-up mutation testing is by leveraging parallel processing. This is an old idea that has not been investigated much. There are many tools supporting parallel execution of mutants, like [147, 204, 205], but they do not report any results or specific advances. Mateo et al. 206] reports results from five algorithms and shows that the mutant execution cost is reduced proportionally to the number of nodes that one is using.

Finally, there are also approaches tackling the problem in specialised cases. For instance, Gligoric et al. [207, 208] suggest a method for the efficient state- 
space exploration of multithreaded programs. This work involves optimisation techniques and heuristics that achieve substantial mutant execution savings. In the context of fault localisation, Gong et al. 209] proposed a dynamic strategy that avoids executing mutants that do not contribute to the computation of mutant suspiciousness and achieves $32.4 \%$ to $87 \%$ cost-reductions. In the case of regression testing, Zhang et al. [210] identifies the mutants that are affected by the program changes (made during regression) and executes only those in order to compute the mutation score. The affected mutants are identified with a form of slicing (dependencies between mutants and program changes). Wright et al. 211. uses mutant schemata and parallelisation to optimise the test of relational database schemas. Zhou and Frankl 212] proposed a technique called inferential checking that determines whether mutants of database updating statements (INSERT, DELETE, and UPDATE) can be killed by observing the state change they induce.

\subsection{Mutation Score Calculation and Refinement (Step 6.)}

The mutant execution aims at determining which mutants are killed and which are not. By calculating this number, we can compute the mutation score that represents the level of the test thoroughness achieved. Determining whether a test execution resulted in killing a mutant requires observing and comparing the program outputs. Thus, depending on what we define as a program output we can have different killing conditions. Usually, what constitutes the program output is determined by the level of granularity that the testing is applied to. Usually in unit testing the program output is defined as the observable (public access) return values, object states (or global variables), exceptions that were thrown (or segmentation faults), and program crashes. In system level, program output constitutes everything that the program prints to the standard/error outputs, such as messages printed on the monitor, behaviour of user interfaces, messages sent to other systems and data stored (in files, databases etc.).

In the case of non-deterministic systems, it is necessary to define mutant killing conditions based on a form of oracle that models the behaviour of the 
obtained outputs. Patrick et al. 213] use pseudo-oracles to test stochastic software. Rutherford et al. 214] use discrete-event simulations (executable specifications) to define assertions and sanity checks that model how "reasonable" are the test execution results (distribution topology, communication failure, and timing) of distributed systems.

Observing and comparing the program outputs often requires a test driver that it is program specific and, thus, researchers usually approximate program outputs by observing a subset of it, usually defined by the test assertions (and program crashes). Alternative techniques involve the use of stubs, oracle data, log messages and internal program states that will be detailed later on, in Section 5.9. Mateo et al. [128] proposed flexible weak mutation, an approach for system level mutation testing that considers mutants as killed when they result in corrupted object states. Object states are checked after the execution of every method call. Wu et al. [104] record execution paths and determine whether mutants cause any deviations from the original program's ones (execution of different paths).

Computing the mutation score requires the removal of equivalent mutants. As already discussed in Section 5.3, identifying equivalent mutants is a manual task that is partially addressed through static heuristics. Since the problem is important, there are some attempts to approximate the mutation score using dynamic heuristics. The idea is that mutants that are not killed by the tests but are capable of causing differences on the program state are likely to be killable [215]. This idea was initially introduced by Grun et al. 215] and, later, studied by the works of Schuler and colleagues [216 218]. Overall, these studies examined several heuristics that measure different types of impact (breaking program invariants, changed return values, altered control-flow and data-flow) and showed that measuring whether mutants cause deviations on the program execution forms the best option.

The use of mutants' impact provides opportunities to define mutant selection and classification strategies. Schwarz et al. 219] defined a mutant selection strategy by selecting a small set of mutants with high impact and diverge lo- 
cations (all over the codebase). Mutant classification provides opportunities to achieve good trade-offs between effectiveness and efficiency. Papadakis and Traon [220, 221] defined such strategies and found that mutant' classification is beneficial when low quality test suites are used.

Other attempts to refine and approximate the mutation score with the use of mutant classification are due to Kintis et al. [222, 223]. Kintis et al. observed that killable mutants are likely to compose a higher order mutant that behaves differently than the first order ones that it is composed of. Based on this observation, a mutant classification strategy that identifies $81 \%$ of the killable mutants with a precision of $71 \%$ was proposed.

\subsection{Reduce/Prioritise Test Cases (Step 7.)}

This step involves the test suite reduction and/or test suite prioritisation. Test reduction refers to the process of removing test cases that are somehow redundant, i.e., test cases that when removed from the test suites do not change the mutation score. Test prioritisation refers to the process of ordering test cases in such a way that mutants are killed as early as possible.

Mutation-based test suite reduction has been suggested by Usaola et al. 224, using a greedy algorithm. The idea is to iteratively select the test cases that kill the maximum number of mutants that were not killed by the previously selected test cases. Hao et al. 225] used mutation to estimate a confidence level for the fault detection loss experienced due to the reduced test suites. Therefore, users can minimise their test suites using structural criteria and get an estimation of the potential fault-detection capability loss based on the mutants.

Shi et al. 193 used mutants to reduce test suites and measured different trade-offs between reduced test suites and fault-detection loss when reduced test suites kill fewer mutants than the original (non-reduced) ones. Similar to this work, Alipour et al. [226] proposed reducing (simplifying) individual tests rather than removing some of them and measured the trade-offs between reductions and fault-detection loss. 
Regarding test case prioritisation, Lou et al. [194] studied two prioritisation schemes; one based on the number of mutants killed and one based on the distribution of the killed mutants and found that prioritising based on the number of killed mutants performs best. Nguyen et al. 227] proposed ordering first the test cases that kill the most mutants in order to support the audit testing of webservice compositions. In their work, they considered only a subset of mutants, which is the ones that do not violate the explicit contract with the service under analysis.

\subsection{Confidence Inspired by Mutation Score (Step 8.)}

The mutation testing process stops when mutation score reaches a userspecified threshold. In theory, this threshold reflects the level of confidence that developers have on the testing performed. Unfortunately, there are very few studies related to this subject, i.e., measuring the relationship between mutation score and fault revelation. Along these lines, Li et al. 52 experimented with mutation adequate test suites (test suites that kill all killable mutants) and showed that these tests reveal more faults than the ones of structural testing criteria. This result is in line with the results of older studies that showed the superiority of mutation testing over other structural test criteria [27, 55].

The most recent studies on the subject are those of Papadakis et al. 41] and Chekam et al. 6] that studied the fault revelation ability of mutation testing. The most important finding of the studies is that the "relationship between strong mutation and fault revelation exhibits a form of threshold behaviour" [6] and that "achieving higher mutation scores improves signicantly the fault detection". This means that there is a strong connection between mutation score and fault revelation only at higher mutation score levels (above a specific threshold). However, below that level, the mutation score is completely disconnected from fault-revelation. In practice, this means that inadequate test suites that fail to reach relatively high mutation scores are vulnerable to noise effects

and testers should not be confident on their testing (based on them). Perhaps more importantly, the same study shows that strong mutation adequate test 
suites are capable of revealing at least $90 \%$ of the program faults [6].

The study of Tengeri et al. 228] suggests that mutation testing forms a good indicator of the expected number of defects in a system (number of real faults reported after the release of the system). Since these defects are those missed by the testing process they can be viewed as quality indicators of the test suite thoroughness.

Generally, it is important to consider the role of equivalent and redundant mutants when studying the relationship between mutation score and fault revelation. In practice, both the existence of equivalent and redundant mutants, makes the evaluation of the exact mutation score value obscure, with the unfortunate effect of overestimating or underestimating the true score [36, 152, 229]. In particular, equivalent mutants tend to reduce the true mutation score, while redundant mutants have mixed effects. Therefore, reliably studying the mutantfault relation requires, to some extend, adequate solutions for these problems.

Overall, we know very little regarding this fundamental aspect of software testing (confidence inspired by mutation score). Studies increasing our understanding on this respect are important and should form one of the main subjects addressed by future research. Similarly, studies addressing the equivalent and redundant mutant problems are also key to this problem.

\subsection{Test Oracles (Step 9.)}

Once we create test inputs and reach the desire level of mutation score, we need to check whether the program under test behaves as expected. Additionally, we need to equip our tests with test oracles that assert the desired behaviour for future use. This is the phase were we actually find faults (when the program does not behave as expected). Unfortunately, in the absence of formal specifications this task is carried out manually.

One of the first attempts to automate this process is due to Fraser and Zeller [171, 187] who used mutants to guide oracle assertion creation. The idea is to check (and assert) the part of the program output that is responsible for killing the mutants. Fraser and Zeller [171, 187] formulate this as a search problem and 
devised an automated approach that generates test assertions. Testers are then asked to validate these assertions. The same method has also been extended to identify relevant pre- and post-conditions suitable for parameterized tests [101, 230. In the same vein, Knauth et al. 231] evaluated the quality of contracts (written in the Java Modeling Language) by mutating them.

The use of mutants in creating test oracles has been a common practice in automated test generation tools. Evosuite 232 adopts this practice for generating test oracles for Java programs. Yoshida et al. [233, 234] use the same method to support test generation for $\mathrm{C} / \mathrm{C}++$ programs. Jahangirova et al. [235, 236] use mutants to detect relevant observed state differences and abstract them into test oracles.

Mutants have also been used to drive the creation of oracle data (a set of variables that should be monitored during testing) [237, 238. In this work, internal program variables are monitored and ranked according to their ability to kill mutants. Similar to the work of Fraser and Zeller, mutants assist the creation and minimisation of the oracle data. Jahangirova et al. 239 uses test generation and mutation testing to assess and improve oracles (code assertions). Additional details regarding test oracles can be found in the survey of Barr et al. 240.

\subsection{Debugging (Step 10.)}

Research on mutation-based debugging has followed two main directions, namely fault localisation and fault fixing. The former refers to the problem of locating the code areas that are responsible for a given failure while the later to the problem of automatically repairing the fault using the available test suite.

\subsubsection{Mutation-based Fault Localisation}

Mutation-based fault localisation was introduce by Papadakis and Le Traon [17, 241 with their work on the Metallaxis method. The underlying idea of Metallaxis is that mutants killed mostly by failing tests have a connection (interaction) with the program defects that caused the program failures. Thus, mutants 
killed mostly by failing tests provide indications regarding the faulty program locations. Empirical results on this approach demonstrated that mutation-based fault localisation is significantly superior to other types of fault localisation techniques, such as spectrum-based fault localisation [17, 242].

Metallaxis was later extended to support mutant reduction techniques, such as selective mutation [125] and has been released as an automated tool called Proteum/FL 204]. The idea of Metallaxis was later extended by Moon et al. [243, who introduce the MUSE method. MUSE works by checking whether mutants turn the failing test cases into passing or not. The difference from Metallaxis is that MUSE does not consider the mutants that are killed (have different outputs from the original program) by failing test cases but still they are not passing.

Other mutation-based fault localisation techniques are those of Zhang et al. 244 who studied fault localisation on the context of evolving programs and localised suspicious program edits. Hong et al. 245, 246] extended MUSE for multiligual programs. Empirical results demonstrated that mutation-based techniques identify the faulty program locations (and edits) as the most suspicious statements. Another work of this type is that of Murtaza et al. [247, 248]. In this work, it was observed that the test execution traces produced by mutants and faults are similar. Musco et al. [249] used mutants to approximate a causal graph. This approach realises the idea of tracking causality in call graphs by exploring the test paths that lead to killing mutants.

\subsubsection{Mutation-based Fault Repair and other Debugging Activities}

Mutation has been used to support program repair activities by Debroy and Wong [250, 251]. These works observe that many faults are fixed by simple syntactic transformations. Therefore, since mutants are simple syntactic transformations, they form potential patch candidates. The advantage of this technique is that it is simple and can be completely automated by a mutation testing tool.

Generally, automated fault repair is a large field of research with many 
specialised applications. Most of the approaches use genetic programming or constraint-based techniques to select and check whether special types of mutants can fix the underlying faults.

One of the first attempts in the area is due to Weimer et al. [18, 252, who used genetic programming with statement deletion, statement insertion, statement replacement and crossover mutant operators in order to support the automated bug fixing. Empirical results demonstrated that this approach can be particularly effective 253. Later, Weimer et al. 254] leveraged mutation testing advances in order to improve the performance of fault fixing. In this work, the duality between mutation testing and fault repair is detailed along with potential opportunities for cross fertilisation between the approaches. Other fault repair approaches combining search and mutation testing are by Tan and Roychoudhury [255], who introduce a regression repair technique that searches for mutants (using 8 types of mutant operators) that fix regression faults.

There are many automated fault repair techniques that use some form of syntactic transformations (can be viewed as specialised higher order mutants) in order perform program repair. For instance, Long and Rinard [256] and Kim et al. 257] use syntactic patterns to perform program repair. However, as these approaches fall outside the scope of the present paper, the interested reader is redirected to a specialised survey on this subject [258].

Other debugging techniques relying on a form of mutation testing is Angelic debugging [259]. The idea of Angelic debugging is to perform a form of data state mutation in order to correct the program execution. The idea is to identify the set of values that can substitute the program state (at runtime) and results in a form of "correct" execution.

\section{Alternative Code-based Mutation Testing Advances}

This section details code-based testing advances that do not conform to the mutation testing process, as depicted in Figure 3. These advances include predictions of the mutation score, gamification of mutation testing, the use of 
search algorithms and diversity-aware testing techniques.

Mutation testing requires performing the mutant execution step which is expensive even when using the test execution optimisations that were discussed in Section 5.5. To deal with this problem, many researchers have proposed the use of alternative proxies to measure the fault revealing potential of test cases. Gligoric et al. [59, 60] found that branch coverage measurements are strongly correlated with mutation scores. Therefore, they argued that branch coverage could be used as an alternative to real faults and mutation faults. Later, Gopinath et al. 62 conducted a large empirical study and found that statement coverage scores correlate strongly with mutation scores. Unfortunately, there are also studies demonstrating that coverage does not correlate well with mutant detection 61.

Zhang et al. 260 built a classification model that predicts the mutants that are killed without executing them. The model relies on a number of features related to mutants, tests and coverage measures and predicts the mutant execution results with a relatively good precision (with over 0.85 precision and recall).

Designing mutation-based tests is a tedious and potentially boring task that most developers try to avoid. As testing requires the involvement of developers, their motivation is crucial. In view of this, the studies of Rojas and Fraser [261] and Rojas et al. 262] suggested making these activities entertaining by gamefying mutation testing. The game includes two main roles, the attacker and the defender. The former aims at creating subtle non-equivalent mutants and the latter at creating test cases to kill these mutants. Overall, this approach helps educating and motivating developers. It can also help crowdsourcing complex tasks, such as test generation and adequacy evaluation 262 .

Search-based mutation testing forms an alternative approach to traditional mutation testing. Instead of selecting mutants from a predefined set of operators, it uses meta-heuristic search techniques to evolve and optimise the generation of higher order mutants [263]. The idea here is to seek tailored mutants that fit to the particular goals of the testers. Thus, search-based techniques can 
be employed in order to search the space of all possible mutants for those that are subtle (mutants that are killed by only few test cases) 264, representative of all mutants [38, and realistic (both semantically and syntactically close to the original program) 72].

There is a number of approaches that utilise mutant optimisation: Jia and Harman study ways to combine first order mutants so that they produce subtle faults [38, 264] for C programs. Harman et al. 265] reports that by using search it is possible to improve test effectiveness (between $5.6 \%$ and $12 \%$ ) while enjoying 15\% improved efficiency (in Java programs). Langdon et al. 72] used grammar-based, bi-objective, strongly typed genetic programming to form realistic mutants for $\mathrm{C}$ programs. Along the same lines, Omar et al. [15] experiment with Java and AspectJ mutants. Their results demonstrate that it is possible to generate subtle higher order mutants [266, 267]. Omar et al. [267, 268] experimented with different ways of combining first order mutants in order to improve the efficiency of the approach. They found that a form of local search

performs best. Wu et al. 269] use higher order mutation to genetically improve the non-functional properties of the program under test. Their approach yields time and memory performance improvements of approximately $18 \%$ and $20 \%$, respectively.

Finally, Shin et al. [270, 271] proposed using mutants as a test suite diversity measure and defined a mutation-based diversity test criterion. The idea of this approach is to construct test cases that can distinguish every mutant from every other mutant. Thus, instead of trying to distinguish the behaviour of the mutants from that of the original program, they proposed to distinguish the behaviour of every mutant from all the others.

\section{Advances beyond Code-based Mutation Testing}

This section presents approaches that use mutants to support software engineering activities other than code-based testing. We first outline those belonging to the model-based testing, and continue with those related to security testing 
and, finally, other applications and testing approaches.

\subsection{Model-based Testing}

An important line of mutation-based research regards its application to model artefacts. Older approaches called it specification-based mutation but the newer ones refer to it as model-based mutation. Here, we briefly discuss these approaches. For a detailed description and discussion on this subject, we point the reader to the specialised survey of Belli et al. 30].

Henard et al. 272] propose the use of mutation to test software product lines. The variability of software product lines and configurable systems is compactly represented by feature models. Therefore, the study of Henard et al. introduces mutant operators that mutate feature models (and their constraints). The idea is to transform the feature model into an equivalent logic formula, which is mutated using logical operators (using a tool called MutaLog [273]). Subsequently, the effectiveness of the selected configurations (to detect conformance faults of feature models) is evaluated based on their ability to detect mutants. In a later study, the feature model mutants were used to assist the automatic repair of feature models [274]. This is achieved by iteratively mutating the model under analysis until it reaches the desirable state.

An advantage of using feature model mutants is that by targeting them, it is possible to generate (automatically) a small set of test configurations. Henard et al. 275] applied this idea using search-based optimisation methods in order to minimize the number of selected configurations and maximize the number of killed mutants. Filho et al. [276, 277] used a multi-objective optimisation approach to achieve several trade-offs between the number of selected configurations and number of killed mutants, their diversity and etc.

Generating feature model mutants with the aim of selecting test configurations also been attempted by Arcaini et al. 278. The difference of this method from that of Henard et al. is that mutants are introduced directly on the feature model under test (instead of the logic formula). The study also reports results from a test configuration generation technique that attempts to kill these mu- 
tants. The same approach was then used to evaluate the conformance of feature models to a software product line [279]. This approach also attempts to automatically detect and remove conformance faults from the feature model, similar to Henard et al. 274]. The difference is that since mutants are applied directly on the feature model the resulting models are expected to be easy to understand.

The study of Trakhtenbrot [280] focuses of testing statechart-based models for reactive systems. This approach is concerned with specific semantics of statechart models that are not aligned with the model's implementation. These semantics are the "zero-time" abstraction and "maximal parallelism", which are the subjects of mutation. Considering the conformance relation of action systems, Aichernig and Jöbstl 281] proposed a technique for encoding the semantics (of action systems) as constrains to be incorporated in the test conformance relations. These relations form the mutant killable conditions. Similarly, Aichernig et al. [282, 283] developed a mutation-based test generation technique for UML state machines.

Devroey et al. 12 introduced the notion of featured model-based mutation analysis, a flexible formalism based on Featured Transition Systems, which enable the optimised generation, configuration and execution of mutants. The main idea behind this approach is to represent the model mutants as products of a Software Product Line [151]. Based on this idea, the authors demonstrate that the technique can speed-up mutant execution up to 1,000 times when compared to other behavioural model mutation approaches. Similarly, Belli and Beyazit 284] propose a mutant generation technique that attempts to limit the introduction of equivalent and duplicated mutants. The same approach aims at optimising the test case execution by avoiding the comparison of the mutants with the original models.

El-Fakih et al. 285] present a mutation-based test case generation technique for Extended Finite State Machines (EFSMs) that evaluates whether the EFSM under test conforms to user defined faults. As part of the technique, the EFSM under test is mutated and test cases able to kill the generated mutants are generated. Another technique, proposed by Su et al. [286], utilises mutated 
GUI models for test case generation of Android applications. This particular approach mutates an auto-generated stochastic GUI model of the application, represented as a FSM, in order to search for better models that will result in different, and potentially better, event sequences compared to the original model. Finally, Aichernig et al. [287] combine property-based testing with model-based mutation testing to generate efficiently test suites that target specific coverage criteria based on EFSMs.

As for code-based mutation, detecting equivalent mutants at the model level is a tricky problem. When considering behavioural models such as automata, this problem can be formulated as a language equivalence problem. Indeed, if two automata accept the same language, then their traces are the same and no test case can distinguish them. Language equivalence is P-SPACE complete but efficient algorithms exist. Devroey et al. 3] compared one of such algorithms with two sorts of simulations: one that is completely random and one that exploits syntactic differences between the models to direct trace generation to infected states. Biased simulations proved to be efficient for strong mutation cased on large models while the exact approach was more interesting for weak mutation.

Belli et al. 108 present a mutation-based technique to test "go-back" functions modelled by pushdown automata. This approach uses mutant operators that affect the transitions, state and stack of pushdown automata. Aichernig and Lorber 288, 289] propose a model-based mutation testing technique for timed automata that tackles the state-space explosion problem caused when unfolding timed automata. The method improves the test execution using the unfolded structure of the original specifications. Larsen et al. 290] builds on this work and further improve its efficiency. Zhou et al. 291] present a specificationbased mutation approach to test safety-critical systems. This method defines mutant operators for the Input Output Symbolic Transition System modelling language and introduce a test case generation technique to create test cases based on these mutants. Adra et al. 292] study the application of mutation to agent-based systems. This approach defines mutant operators to address the 
properties of this type of systems.

Stephan et al. [293, 294] present a technique that compares model-clone detection techniques for Simulink models using mutation. This approach introduces a set of structural mutant operators designed to compare model-clone detectors. The design of the operators was based on the authors' observations of potential model edit operations in publicly available models. In an extension of this work, Stephan et al. 295. present a taxonomy of Simulink mutant operators that represent realistic edit scenarios when modelling. Although this taxonomy was created with the comparison of model-clone detectors in mind, the authors suggest that it can represent Simulink model mutations in general. Later, Pill et al. 296 developed a mutation testing framework for Simulink models, named SIMULTATE.

Testing model transformations using mutation has been attempted by Khan and Hassine 297. In this approach the authors introduce specific mutant operators for the Atlas Transformation Language. Later, Troya et al. 298 focuses on the same subject and presented an extensive set of mutant operators that uses both first and higher-order mutation transformations [299]. Another study that tests model transformations using mutation is due to Aranega et al. [300, who focuses on how to support the generation of mutation-adequate test cases for checking model transformations. In this case, test cases are test models. The intuition behind the approach is that building a test model that is able to kill alive mutants from scratch is difficult, thus, the approach attempts to provide guidance to select some of the already available test models (test cases) to modify to kill the alive mutants.

Bartel et al. 301 focus on testing Dynamically Adaptive Systems. These systems are governed by adaptation policies that incorporate how and when the system will adapt. The approach focuses on testing whether these adaptation policies are correctly implemented using mutation. Thus, a set of mutant operators is defined using a meta-model that represent the policy formalisms. The approach also suggests a specialisation of these mutant operators for the case of action-based adaptation policies. 
Mutation testing has also been applied on NuSMV models. Arcaini et al. 302 creates mutants of such models and checks whether the NuSMV model advisor (an automatic static model review tool) can statically detect these mutants. Mutant operators for UML domain models have been defined by Kaplan et al. 303. This study aims at generating test cases based on information provided by the domain model, expressed as a UML class diagram with invariants, and the use case model of the application under test. Fraser and Wotawa 304 presents another model-based mutation approach aiming at determining property violations of a model. The approach relies on the notion of property relevance which relates test cases to model properties, in an attempt to connect the failing of a test case with a violation of a property.

The application of mutation testing at system requirements that are expressed in a natural language has also been attempted. Trakhtenbrot [305] introduced a semantic mutation approach that introduces mutants related to the intended meaning of the requirements (requirements expressed by predifined patterns) by altering the pattern of the requirements. This enables the use of the mutants for test generation and test evaluation.

Mutation testing has also been applied on Alloy models. Sullivan et al. 306, performed mutation testing in declarative programming paradigm (Alloy language) to support test case generation and showed that it is robust at revealing real faults.

Finally, mutation has been applied on aspect-oriented programs by mutating state models. Xu et al. 307] proposes two strategies for generating tests from such models. The first one leverages structural information from the state model to generate test cases whereas the second one is based on counterexamples generated by model-checking (counterexamples that form illegal sequences of events in the original model). The study of Lindström et al. 308 introduces a mutation-based approach to test aspect-oriented models. The approach proposes a set of mutant operators targeting specific features of aspect-oriented modelling. Abstract tests created to kill the generated mutants evaluate the modelling of cross-cutting concerns and the weaving process, as well. 


\subsection{Security Testing}

This section presents mutation-based approaches related to security. More precisely, applications on testing security policies [309 313, regression testing of security policies [314] and testing security protocols [315] are shortly described.

Testing security policies using mutation has been suggested by Mouelhi et al. 310. In this study, Mouelhi et al. propose a meta-model that captures different rule-based security policy formalisms. This meta-model forms the subject for mutation which is performed using a set of (proposed) generic operators that can simulate faults in the various instantiations of the model. Along the same lines, Mouelhi et al. 312 present another mutation-based technique that automatically transforms functional test cases into security test cases (test the security policy). In this approach, mutation is used for two purposes: to identify the subset of the functional test cases that are impacted by the security policy and to relate this subset's functional test cases to specific security policy rules.

Dadeau et al. 315, 316] propose a mutation-based test generation and evaluation technique that validates an implementation of a security protocol that is written in the High-Level Security Protocol Language. These approaches propose mutant operators that introduce leaks in the security protocols and creates abstract test cases for HLPSL models by targeting/killing mutants.

Other attempts to test security policies are due to Bertolino et al. [311] who propose a fault model for history-based security policies. This study aims at policies written in the PolPA language and proposes modification rules that attempt to simulate faults that can occur in the implementation of the Policy Decision Point (PDP) and target only the static behaviour of the PDP. Elrakaiby et al. 309] and Nguyen et al. 313] attempt to test the obligation policy enforcement and delegation policies. These goals are achieved by using mutant operators that introduce changes in key elements of the obligation policy management and delegation features.

Finally, Hwang et al. 314] investigate test selection techniques for regression testing of security policies. They proposed three techniques towards this goal, one of which is based on mutation analysis. This particular technique first uses 
mutation analysis to correlate policy rules and tests cases and, subsequently, it applies test selection. The test selection is performed by selecting test cases that are correlated with rules involved with syntactic changes between the original policy and its mutants.

\subsection{Supporting adaptive random testing, boundary value analysis and combina-} torial interaction testing

Mutation testing has been used to support or extend several not mutationbased testing methods. Thus, it has been used to support adaptive random testing, boundary value analysis and combinatorial interaction testing.

In the context of combinatorial interaction testing, Papadakis et al. 317. proposed mutating the constraints between the program input parameters. Thus, instead of selecting input combinations that satisfy the input constraints only, the authors proposed selecting the combinations that make the mutated constraints invalid. The underlying idea of this approach is that the difference between the original and the mutant constraints define some form of 'boundary' conditions that trigger faults. Empirical results with faulty applications demonstrated that mutants have a stronger correlation with faults than the input parameter combinations.

Zhang et al. 318 proposed a mutation-based extension to boundary value analysis. The approach mutates some predicates of a given path condition in order to define boundary values. Similarly to Papadakis et al. [317, these values are the solutions that satisfy the path condition and at the same time differentiate the original predicate from its mutants. The authors also propose a way to generate test cases that cover these boundary conditions based on constrained combinatorial interaction testing.

Patrick and Jia 319, 320, proposed a technique, named Kernel Density, to support adaptive random testing. This technique guides the test selection process based on the killed mutants. Thus, tests killing new mutants are considered to be more distant than those killing the same ones. Empirical results show that test cases selected by this approach kill more mutants than the ones selected by 
adaptive random testing.

\subsection{Other Mutation-based Applications}

This section describes approaches tackling general software engineering problems. These include: program analysis, software verification, code clones, defect prediction and regression testing.

Mutation analysis has been used to automatically detect loop invariants by mutating postcondition clauses 321. In such a way, many invariant candidates are generated and invalid invariants are discarded based on appropriate counterexamples. The study of Galeotti et al. [321] describes several ways to mutate the postconditions, as well as, ways to eliminate some trivial cases. Similarly, Andrés et al. 322 propose an automated framework, named PASTE, that uses mutants to evaluate the fault revealing ability of system invariants (generated from specifications) in the context of passive testing of stochastic timed systems. The approach evaluates the strengths of the invariants (and prioritises them) based on the number of killed mutants. Subsequent works detail (its mutation module, its mutant operators and the algorithms it incorporates), extend and evaluate the framework further [323, 324].

Pankumhang et al. 325 propose a code instrumentation technique, named iterative instrumentation, for measuring code coverage when testing time-sensitive systems. The approach is based on weak mutation analysis and instruments the program by inserting exit statements at the instrumentation points considered.

Other applications of mutation testing include its use for software verification. Groce et al. 326 used mutants to make developers familiar with software verification. The idea is to focus on incorrect programs (mutants) in order to understand when and how the verification process fails (by observing failures to detect problems caused by mutants).

Using mutation analysis to create software clones forms another application of mutation. Roy and Cordy 294] introduce several mutant operators that model typical copy/paste activities of developers and create clones based on the

application of these operators. The same study also uses these clones to evaluate 
different clone detection techniques. Along these lines, the work of Svajlenko et al. 327. presents mutant operators that create fork constructs in order to assist the study and analysis of code similarity.

More recently, Bower et al. 328, proposed using mutation to assist the prediction of software defects. This technique combines traditional source code metrics with a number of mutation analysis metrics to built defect classifiers. The mutation analysis metrics that were used are classified into static, e.g. the number of mutants a mutant operator generated, and dynamic ones, e.g. the number of mutants killed. The study concludes that mutation-based metrics significantly improve the performance of defect prediction and that the best results are obtained by using a combination of static and dynamic metrics.

In the context of regression testing, Zhang et al. 329] used special forms of mutants to improve the fault detection ability of regression test suites. The approach mutates both the old and the new versions of the program under test, and executes them with the available test suites. The detected differences between the two versions are considered as problems.

Di Nardo et al. [330] present a mutation-based technique to automatically generate faulty input data within complex data structures from existing field data. The approach uses six generic mutant operators that mutate the field data and guide their selection (using a data model). Results from an industrial case study show that it performs better, in terms of code coverage, than the manual testing performed by domain experts.

As discussed earlier, the existence of equivalent mutants constitutes one of the major costs of mutation's application. However, many researchers have started viewing this as an advantage in certain cases. Arcaini et al. 331 , 332 seek to find opportunities to improve the quality of the artefact under consideration. For example, they suggest that equivalent mutants can be used for improving code readability and for refactoring purposes. It is suggested that benefits from equivalent mutants may arise on all software artefacts where mutation applies. For instance, in feature models it is possible to detect dead and false optional features and redundant constraints. A similar approach is 
that of Baudry et al. [333, who suggested that equivalent mutants can be seen as diverse program versions. Therefore, by generating equivalent program versions, one can produce multiple diverse program variants (which can support security purposes, like moving target defense).

Lisper et al. [334] introduced the concept of targeted mutation, which aims at non-functional properties. The idea undelying this approach is to introduce mutants that are relevant to a targeted non-functional property and use them as guides for generating and augmenting test suites. These test suites can then be used for estimating the worst-case execution time.

Finally, another mutation-based testing technique refers to testing relational database schemas. Wright et al. 211] investigate ways to make the application of mutation to database schemas more efficient. To this end, the authors propose and evaluate 4 cost-reduction approaches that leverage mutant schemata and parallelisation. The results of the empirical study conducted suggest that the mutation analysis time can be reduced by the approaches proposed but they also indicate that their performance can be influenced by the underlining database management system (DBMS).

\section{Tools for Mutation Testing}

One important factor for the successful application of mutation is the availability of automated frameworks that support its application steps. This section discusses the tools that were introduced or were used in the studies we surveyed. Table 3 outlines the corresponding tools along with the year of their creation, their application artefact and a concise description of key characteristics.

As it can be seen from the table, our analysis concluded in 76 tools, most of which where introduced between 2008 and 2017, that apply mutation to different software artefacts. By closely examining the table, it becomes obvious that there is an increasing growth in mutation testing tools with the creation of approximately 10 tools per year. Most of these tools target the implementation level languages but there are also tools that target specification languages and 
models.

At the implementation level, the mutation testing tools target mostly the $\mathrm{C}$ and Java programming languages. Most of the tools focus on the support of traditional, method-level mutant operators and strong mutation, with few tools supporting Object-Oriented operators and weak or higher order mutation. Additionally, there have been various tools proposed that apply mutation to dynamically-typed programming languages and concurrency-related aspects.

For the non-code-based tools, there have been proposed various tools for many model notations, including Extended and Timed Finite State Machines, Simulink models, Feature Models, etc, that automate the application of mutation. Furthermore, automated frameworks for mutating security policies and protocols have also been introduced.

Table 3: Mutation Testing Tools.

\begin{tabular}{|c|c|c|c|}
\hline Name \& Ref & Year & Application & Description \\
\hline MUTATE 335 & $\mathrm{~N} / \mathrm{A}$ & $\mathrm{C}$ & supports method-level mutant operators \\
\hline JESTER 336 & 2001 & Java & $\begin{array}{l}\text { supports source-code-level (src-level) mu- } \\
\text { tant generation }\end{array}$ \\
\hline Proteum 337 & 2001 & $\mathrm{C}$ & $\begin{array}{l}\text { supports an extensive set of method-level } \\
\text { mutant operators and interface mutation } \\
\text { (inter-method level operators) }\end{array}$ \\
\hline MUTGEN 338339 & 2003 & $\mathrm{C}$ & supports method-level mutant operators \\
\hline $\begin{array}{llll}\text { MUJAVA } & 9 & 340,341 \\
\end{array}$ & 2004 & Java & $\begin{array}{l}\text { implements src-level mutant generation } \\
\text { and supports method-level and Object- } \\
\text { Oriented (OO) mutant operators }\end{array}$ \\
\hline BYTEME 342 & 2006 & Java & $\begin{array}{l}\text { implements bytecode-level mutant gen- } \\
\text { eration and supports method-level \& } \\
\text { Object-Oriented (OO) mutant operators }\end{array}$ \\
\hline SQLMUTATION 343 & 2006 & SQL & $\begin{array}{l}\text { supports traditional \& SQL-specific mu- } \\
\text { tant operators for SQL queries }\end{array}$ \\
\hline Jumble 344 & 2007 & Java & $\begin{array}{l}\text { implements bytecode-level mutant gener- } \\
\text { ation and supports method-level mutant } \\
\text { operators }\end{array}$ \\
\hline ESTP 345 & 2008 & $\mathrm{C}$ & $\begin{array}{l}\text { supports } 20 \text { traditional } \mathrm{C} \text { mutant opera- } \\
\text { tors }\end{array}$ \\
\hline Not Named 346 & 2008 & Sulu & $\begin{array}{l}\text { supports method-level mutant operators } \\
\text { (drawn from the study of Andrews et al. } \\
339 \text { ) }\end{array}$ \\
\hline MiLU 347 & 2008 & $\mathrm{C}$ & $\begin{array}{l}\text { supports method-level mutant operators } \\
\text { and higher order mutation }\end{array}$ \\
\hline
\end{tabular}


Table 3: Mutation Testing Tools.

\begin{tabular}{|c|c|c|c|}
\hline Name \& Ref & Year & Application & Description \\
\hline Not Named 304 & 2008 & $\begin{array}{l}\text { NuSMV } \\
\text { models }\end{array}$ & $\begin{array}{l}\text { supports specification-based mutation } \\
\text { (drawn from the study of Black et al. [2]) }\end{array}$ \\
\hline Not Named 348 & 2008 & $\begin{array}{l}\text { Code gen- } \\
\text { erated from } \\
\text { Simulink } \\
\text { models }\end{array}$ & $\begin{array}{l}\text { seeds faults into the implementations } \\
\text { generated from Simulink models }\end{array}$ \\
\hline Not Named 310 & 2008 & $\begin{array}{l}\text { Security } \\
\text { Policies }\end{array}$ & $\begin{array}{l}\text { supports security-policy-access-control } \\
\text { meta-model mutation (applied on poli- } \\
\text { cies defined in various notations (e.g. } \\
\text { RBAC and OrBAC) }\end{array}$ \\
\hline Not Named 349 & 2008 & $\begin{array}{l}\text { LOTOS } \\
\text { specifica- } \\
\text { tions }\end{array}$ & $\begin{array}{l}\text { supports mutation testing for LOTOS } \\
\text { specifications }\end{array}$ \\
\hline Not Named 77 & 2008 & AspectJ & $\begin{array}{l}\text { supports mutant operators for the cre- } \\
\text { ation of pointcut mutants that vary the } \\
\text { strength of the corresponding point-cut } \\
\text { in terms of the number of joint points it } \\
\text { matches }\end{array}$ \\
\hline JAVALANCHE 205 & 2009 & Java & $\begin{array}{l}\text { implements bytecode-level mutant gener- } \\
\text { ation and supports method-level mutant } \\
\text { operators and mutant classification based } \\
\text { on mutants' impact }\end{array}$ \\
\hline JDAMA 350,351 & 2009 & SQL/JDBC & $\begin{array}{l}\text { implements bytecode-level mutant gener- } \\
\text { ation and supports SQL-related opera- } \\
\text { tors and weak mutation }\end{array}$ \\
\hline $\begin{array}{l}\text { AJMutator } \\
352,353\end{array}$ & 2009 & AspectJ & $\begin{array}{l}\text { supports mutant operators for AspectJ } \\
\text { Point-cut Descriptors (PCDs) } 84 \text { and } \\
\text { automated equivalent mutant detection }\end{array}$ \\
\hline GAMERA 354 & 2009 & WS-BPEL & $\begin{array}{l}\text { supports mutation testing for WS-BPEL } \\
\text { composition }\end{array}$ \\
\hline Not Named 124 & 2009 & $\begin{array}{l}\text { boolean } \\
\text { logic }\end{array}$ & $\begin{array}{l}\text { supports mutant operators for possible } \\
\text { DNF logic faults }\end{array}$ \\
\hline PASTE 322,324 & 2009 & TFSM & $\begin{array}{l}\text { supports passive testing of systems pre- } \\
\text { senting stochastic-time information using } \\
\text { mutant operators specific to Timed Fi- } \\
\text { nite State Machices (TFSM) }\end{array}$ \\
\hline Not Named 355 & 2009 & $\mathrm{Z}$ & $\begin{array}{l}\text { supports mutant operators for } \mathrm{Z} \text { specifi- } \\
\text { cations }\end{array}$ \\
\hline Not Named 356 & 2009 & GCC-XML & $\begin{array}{l}\text { supports a subset of mutant operators } \\
\text { proposed by Ellims et al. } 357\end{array}$ \\
\hline Not Named 358 & 2009 & $\begin{array}{l}\text { LUSTRE / } \\
\text { SCADE }\end{array}$ & $\begin{array}{l}\text { supports mutant operators for LUS- } \\
\text { TRE/SCADE programs }\end{array}$ \\
\hline
\end{tabular}


Table 3: Mutation Testing Tools.

\begin{tabular}{|c|c|c|c|}
\hline Name \& Ref & Year & Application & Description \\
\hline Not Named 231 & 2009 & Java & $\begin{array}{l}\text { supports mutant operators that follow } \\
\text { the fault classification of Durães and } \\
\text { Madeira } 359\end{array}$ \\
\hline PIT 147 & 2010 & Java & $\begin{array}{l}\text { implements bytecode-level mutant gener- } \\
\text { ation and supports method-level mutant } \\
\text { operators }\end{array}$ \\
\hline MutMut 208 & 2010 & Java & $\begin{array}{l}\text { supports concurrency-related mutant op- } \\
\text { erators }\end{array}$ \\
\hline GenMutants 182 & 2010 & .Net & supports method-level mutant operators \\
\hline JUDY 360 & 2010 & Java & $\begin{array}{l}\text { implements src-level mutant generation } \\
\text { and supports method-level \& OO mutant } \\
\text { operators }\end{array}$ \\
\hline WEBMuJaVA 88 & 2010 & HTML/JSP & $\begin{array}{l}\text { supports specific mutant operators for } \\
\text { web components written in HTML and } \\
\text { JSP languages }\end{array}$ \\
\hline BACTERIO [5] & 2010 & Java & $\begin{array}{l}\text { supports method-level mutant operators } \\
\text { for system-level testing using flexible } \\
\text { weak mutation }\end{array}$ \\
\hline $\begin{array}{l}\text { Not Named } 141 \\
177,181,188,190 \\
\end{array}$ & 2010 & & supports method-level mutant operators \\
\hline MAJOR 361 & 2011 & Java & supports method-level mutant operators \\
\hline PARA $\mu$ 362. & 2011 & Java & $\begin{array}{l}\text { supports OO \& concurrency-related mu- } \\
\text { tant operators and higher order mutation }\end{array}$ \\
\hline ILMutATOR 8 & 2011 & $\mathrm{C \#}$ & $\begin{array}{l}\text { implements CLI-level mutant generation } \\
\text { and supports method-level \& OO mutant } \\
\text { operators }\end{array}$ \\
\hline SMUTANT 86 & 2011 & Smalltalk & $\begin{array}{l}\text { supports traditional, method-level mu- } \\
\text { tant operators in a dynamically typed } \\
\text { language }\end{array}$ \\
\hline MUBPEL 363 & 2011 & WS-BPEL & $\mathrm{N} / \mathrm{A}$ \\
\hline JMUHLPSL 315 & 2011 & HLPSL & $\begin{array}{l}\text { supports mutant operators that intro- } \\
\text { duce leaks in security protocols }\end{array}$ \\
\hline Not Named 364 & 2011 & SPADE & $\begin{array}{l}\text { mutates the flow pattern description } \\
\text { of input and output streams and the } \\
\text { SPADE code of components }\end{array}$ \\
\hline Not Named 365 & 2011 & Aglets & $\begin{array}{l}\text { supports mutant operators specific to } \\
\text { Mobile Agent Systems that affect the } \\
\text { movement, communication, run method, } \\
\text { creation, event listeners and agent proxy } \\
\text { of an agent }\end{array}$ \\
\hline Not Named 366 & 2011 & Java & $\begin{array}{l}\text { supports method-level mutant operators } \\
\text { based on the selective mutation approach } \\
\text { and higher order mutation }\end{array}$ \\
\hline
\end{tabular}


Table 3: Mutation Testing Tools.

\begin{tabular}{|c|c|c|c|}
\hline Name \& Ref & Year & Application & Description \\
\hline SMT-C 367 & 2012 & $\mathrm{C}$ & $\begin{array}{l}\text { supports the semantic-related and } \\
\text { method-level mutant operators }\end{array}$ \\
\hline $\begin{array}{l}\text { MUTANT (MURUBY) } \\
\text { 11, 368 }\end{array}$ & 2012 & Ruby & supports Ruby-specific mutant operators \\
\hline Not Named 309 & 2012 & $\begin{array}{l}\text { Obligation } \\
\text { Policies }\end{array}$ & $\begin{array}{l}\text { supports mutant operators specific to } \\
\text { obligation policy enforcement }\end{array}$ \\
\hline Not Named 149 & 2012 & & $\begin{array}{l}\text { supports traditional, method-level mu- } \\
\text { tant operators }\end{array}$ \\
\hline CCMUTATOR 369 & 2013 & $\mathrm{C} / \mathrm{C}++$ & $\begin{array}{l}\text { supports concurrency-related mutant op- } \\
\text { erators, higher order mutation and } \\
\text { targets applications written using the } \\
\text { PThreads and } \mathrm{C}++11 \text { concurrency con- } \\
\text { structs }\end{array}$ \\
\hline Comutation 123 & 2013 & Java & $\begin{array}{l}\text { supports concurrency-related mutant op- } \\
\text { erators } 370\end{array}$ \\
\hline $\begin{array}{l}\text { SCHEMAANALYST } \\
371\end{array}$ & 2013 & SQL & $\begin{array}{l}\text { supports mutant operators related to re- } \\
\text { lational schema integrity constraints, ap- } \\
\text { plied to multiple database management } \\
\text { systems }\end{array}$ \\
\hline XACMUT 372 & 2013 & XACML & $\begin{array}{l}\text { supports mutant operators targeting } \\
\text { XACML } 2.0 \text { security policies }\end{array}$ \\
\hline MUTANDis [10, 81 & 2013 & JavaScript & $\begin{array}{l}\text { supports JavaScript-specific mutant op- } \\
\text { erators }\end{array}$ \\
\hline Not Named 373 & 2013 & $\begin{array}{l}\text { Web service } \\
\text { composi- } \\
\text { tions }\end{array}$ & $\begin{array}{l}\text { supports } 2 \text { types of mutant operators for } \\
\text { web service compositions: one that is in- } \\
\text { ternal to the service and one that models } \\
\text { inconsistencies across different services of } \\
\text { the composition }\end{array}$ \\
\hline Not Named 313 & 2013 & $\begin{array}{l}\text { Security } \\
\text { Policies }\end{array}$ & $\begin{array}{l}\text { supports mutant operators specific to } \\
\text { delegation policies based on a formal } \\
\text { analysis of key delegation features }\end{array}$ \\
\hline Not Named 272 & 2013 & $\begin{array}{l}\text { Feature } \\
\text { Models }\end{array}$ & $\begin{array}{l}\text { supports mutant operators for mutating } \\
\text { Feature Models }\end{array}$ \\
\hline MutPy 374 & 2014 & Python & $\begin{array}{l}\text { implements traditional and python- } \\
\text { specific mutation operators }\end{array}$ \\
\hline MUCHECK 14 & 2014 & Haskell & $\begin{array}{l}\text { supports mutant operators targeting } \\
\text { functional constructs and higher order } \\
\text { mutation }\end{array}$ \\
\hline HOMAJ 375 & 2014 & AspectJ/Java & supports higher order mutation \\
\hline Not Named 376 & 2014 & HTML/CSS & $\begin{array}{l}\text { supports mutant operators that seed pre- } \\
\text { sentation defects to web pages }\end{array}$ \\
\hline
\end{tabular}


Table 3: Mutation Testing Tools.

\begin{tabular}{|c|c|c|c|}
\hline Name \& Ref & Year & Application & Description \\
\hline Not Named 377 & 2014 & EFSM & $\begin{array}{l}\text { supports mutants that introduce Sin- } \\
\text { gle Transfer Faults (STFs) and Double } \\
\text { Transfer Faults (DTFs) to Extended Fi- } \\
\text { nite State Machices (EFSM) models }\end{array}$ \\
\hline Not Named 378 & 2014 & $\begin{array}{l}\text { Data flow } \\
\text { languages }\end{array}$ & $\begin{array}{l}\text { supports } 2 \text { mutant operators that model } \\
\text { common mistakes when creating power } \\
\text { plant control programs }\end{array}$ \\
\hline MutaLog 273 & 2014 & $\begin{array}{l}\text { Logic Muta- } \\
\text { tion }\end{array}$ & $\begin{array}{l}\text { supports mutant operators for mutating } \\
\text { logic expressions }\end{array}$ \\
\hline REDECHECK 379] & 2015 & HTML/CSS & $\begin{array}{l}\text { supports mutant operators for layout de- } \\
\text { fects in responsive web sites }\end{array}$ \\
\hline Not Named 380. & 2015 & spreadsheets & $\begin{array}{l}\text { supports mutant operators for spread- } \\
\text { sheets (spreadsheet mutation) }\end{array}$ \\
\hline Not Named 381 & 2015 & FSM & $\begin{array}{l}\text { supports mutant operators for FSM spec- } \\
\text { ifications (based on the studies of Fabbri } \\
\text { et al. } 382 \text { and Petrenko et. al } 383 \text { ) }\end{array}$ \\
\hline Not Named 384 & 2015 & $\begin{array}{l}\text { Component- } \\
\text { level se- } \\
\text { quence } \\
\text { and state } \\
\text { diagrams }\end{array}$ & $\begin{array}{l}\text { supports architecture- and design-level } \\
\text { mutant operators }\end{array}$ \\
\hline Not Named 385 & 2015 & $\begin{array}{l}\text { HTML / } \\
\text { JavaScript }\end{array}$ & $\begin{array}{l}\text { supports mutant operators for the Model- } \\
\text { View-Controller frameworks of web ap- } \\
\text { plication development }\end{array}$ \\
\hline $\begin{array}{l}\text { Not } \quad \text { Named } \\
103,104\end{array}$ & 2015 & $\mathrm{C}$ & $\begin{array}{l}\text { supports memory-related mutant opera- } \\
\text { tors that model memory faults and con- } \\
\text { trol flow deviation as a mutant-killing } \\
\text { condition }\end{array}$ \\
\hline Not Named 91 & 2015 & $\begin{array}{l}\text { Android } \\
\text { apps }\end{array}$ & $\begin{array}{l}\text { supports android-specific mutant opera- } \\
\text { tors, affecting intents, events, activity } \\
\text { lifecycle and XML files; and the method- } \\
\text { level mutant operators of MUJAVA }\end{array}$ \\
\hline MoMut 386 & 2015 & $\begin{array}{l}\text { UML mod- } \\
\text { els }\end{array}$ & $\begin{array}{l}\text { supports model-based mutation testing } \\
\text { for UML state charts, class diagrams and } \\
\text { instance diagrams }\end{array}$ \\
\hline MuVM 143 & 2016 & $\mathrm{C}$ & $\begin{array}{l}\text { implements bitcode-level mutant genera- } \\
\text { tion and supports higher order mutation }\end{array}$ \\
\hline Not Named 387 & 2016 & FBD & $\begin{array}{l}\text { supports mutant operators for FBD lan- } \\
\text { guage }\end{array}$ \\
\hline Not Named 388 & 2016 & Simulink & $\begin{array}{l}\text { supports mutant operators that model } \\
\text { common Simulink fault patterns }\end{array}$ \\
\hline Not Named 233 & 2016 & $\mathrm{C}++$ & $\begin{array}{l}\text { supports mutant operators similar to the } \\
\text { ones of PIT for the Java language }\end{array}$ \\
\hline
\end{tabular}


Table 3: Mutation Testing Tools.

\begin{tabular}{|c|c|c|c|}
\hline Name \& Ref & Year & Application & Description \\
\hline Not Named 148 & 2016 & $\mathrm{C}$ & $\begin{array}{l}\text { implements LLVM-level mutant genera- } \\
\text { tion and supports method-level mutant } \\
\text { operators typically used in other tools, } \\
\text { e.g. MILU }\end{array}$ \\
\hline Not Named 36 389 & 2016 & $\mathrm{C}$ & $\begin{array}{l}\text { supports traditional, method-level mu- } \\
\text { tant operators }\end{array}$ \\
\hline Not Named 242 & 2016 & & $\begin{array}{l}\text { supports traditional, method-level mu- } \\
\text { tant operators }\end{array}$ \\
\hline Vibes 12, 390 & 2016 & $\begin{array}{l}\text { Transition } \\
\text { Systems, } \\
\text { Statechart } \\
\text { Models }\end{array}$ & $\begin{array}{l}\text { Implements featured model-based muta- } \\
\text { tion analysis and supports the Fabbri et } \\
\text { al. } 382 \text { operator set (both first order } \\
\text { and higher order) }\end{array}$ \\
\hline$\mu$ Droid 110 & 2017 & $\begin{array}{l}\text { Android } \\
\text { apps }\end{array}$ & $\begin{array}{l}\text { Implements energy-aware mutation oper- } \\
\text { ators derived from a specific energy de- } \\
\text { fect model }\end{array}$ \\
\hline MDroid +94 & 2017 & $\begin{array}{l}\text { Android } \\
\text { apps }\end{array}$ & $\begin{array}{l}\text { Implements mutation operators to } \\
\text { test Android applications based on } \\
\text { a specifically-designed Android fault } \\
\text { model derived from manual analysis of } \\
\text { various software artefacts }\end{array}$ \\
\hline Not named 102 & 2017 & $\begin{array}{l}\text { Source } \\
\text { Code }\end{array}$ & $\begin{array}{l}\text { Extracts mutation operators from } \\
\text { changes made in the development his- } \\
\text { tory of projects in an attempt to produce } \\
\text { more "realistic" mutants }\end{array}$ \\
\hline LittleDarwin 391 & 2017 & Java & $\begin{array}{l}\text { Supports method-level mutation oper- } \\
\text { ators, higher order mutation, mutant } \\
\text { sampling and disjoint/subsuming mutant } \\
\text { analysis }\end{array}$ \\
\hline MuCPP 7 & 2017 & $\mathrm{C}++$ & $\begin{array}{l}\text { Implements class-level, object-oriented } \\
\text { mutation operators for } \mathrm{C}++ \text { programs }\end{array}$ \\
\hline MutRex 111 & 2017 & $\begin{array}{l}\text { Regular Ex- } \\
\text { pressions }\end{array}$ & $\begin{array}{l}\text { Implements mutation operators based on } \\
\text { a specific fault model for regular expres- } \\
\text { sions }\end{array}$ \\
\hline BacterioWeb 93 & 2017 & $\begin{array}{l}\text { Android } \\
\text { apps }\end{array}$ & $\begin{array}{l}\text { Implements mutation operators for An- } \\
\text { droid applications }\end{array}$ \\
\hline Not Named 6 & 2017 & $\mathrm{C}$ & Supports method-level mutant operators \\
\hline Not Named 142 & 2017 & $\mathrm{C}$ & $\begin{array}{l}\text { Implements method-level mutation oper- } \\
\text { ators and the AccMut approach } 142 \text { to } \\
\text { reduce the cost of mutant execution }\end{array}$ \\
\hline Not Named 19 & 2017 & Java & $\begin{array}{l}\text { Implements security-aware mutation op- } \\
\text { erators }\end{array}$ \\
\hline
\end{tabular}




\section{Mutation-based Test Assessment: Use and Threats to Validity}

Mutation testing is a popular technique for assessing the fault revealing potential of test suites. Much work on empirical software engineering relies on the use of artificial faults (mutants or manually seeded faults). Researchers employ mutants to perform controlled experiments and assess the relative strengths of test strategies. We call this practice as mutation-based test assessment.

A typical mutation-based test assessment scenario arises when we want to determine whether one method, say Method-1, is more effective than another one, say Method-2. For instance, suppose that the methods to compare are a random test generation (Method-1) and a search-based test generation (Method2 ). In this case, our objective is to check whether one of them has a higher fault revealing ability within a given amount of time. This is assessed by counting the number of killed mutants, i.e., the technique that kills the highest number of mutants is the winning one. Of course, in this particular case, the approaches are stochastic and thus, the experiment needs to be repeated multiple times and assessed by a statistical test, but in every case the technique that kills statistically significant more mutants is the winning one.

This is an intuitive choice made by many empirical studies. However, is it safe to conclude that Method-1 which kills more mutants than Method-2 is better? Actually, it is hard to draw any such conclusion unless we carefully consider and control a number of parameters. As we shall discuss in this section, there are influential factors lying at the heart of mutation-based test assessment that can hamper our ability to assess the fault-revealing potential of the techniques.

\subsection{The use of Mutation in Empirical Studies}

Using mutants as an effectiveness metric is a common practice, which previous research suggests that is adopted by more than a quarter of software testing controlled experiments [36]. To demonstrate the importance and popularity of this practice, we collected the papers that conduct empirical studies and use mutation testing as an assessment method and we analyse them to identify: 


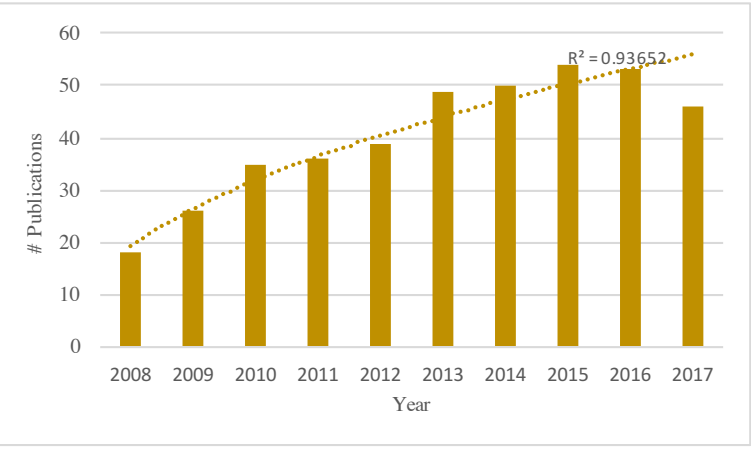

Figure 5: Number of empirical studies using mutation testing as an assessment method.

1) how often mutation testing is used as an assessment method; 2) the types of assessment that are used; 3) the tools that are frequently used; and, 4) the languages mutation is applied to.

The results of our analysis show that, in most cases, mutation was applied to evaluate test techniques. Thus, mutants are used as proxies for real faults and the mutation score is used as an indicator of real fault detection. Figure 5 presents the distribution of these studies in a yearly basis, including the overall growth trend. It is clear from the figure that the application of mutation testing, to experimental studies has been steadily increasing over the last 10 years.

It is important to note that many of the analysed studies are strictly not concerned with mutation testing; their objectives do not include mutation-related software engineering problems. Rather, mutation is a mechanism to validate the study and not the subject of the study.

Overall, from the papers we surveyed, we identified 190 papers falling into this category. Taking these findings into account, we can conclude that an increasing number of scientific results rely on mutation.

Figure 6 presents the types of mutation-based test assessment. As can be seen from the figure, mutation's primary use in experimental studies is for comparing test techniques, Comparison Basis. The second largest category refers to Test Assessment and includes the test effectiveness of single techniques 


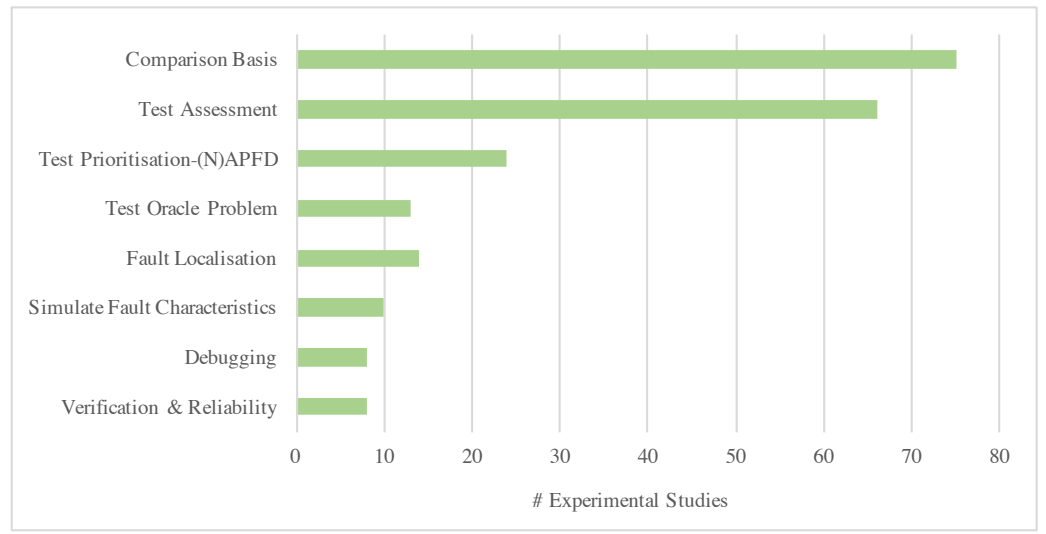

Figure 6: Different types of mutation-based test assessment.

(without comparison), i.e., a test technique kills this number of mutants. The other categories involve assessments for test prioritisation (Test Prioritisation(N)APFD $]^{2}$ test oracle (Test Oracle Problem), localisation of faults (Fault Localisation), verification techniques (Verification $\mathscr{E}$ Reliability), debugging (Debugging) and other techniques.

Figure 7 depicts the mutation testing tools that were used in the experimental studies along with the number of studies using these tools. It is noted that the figure includes only the most frequently-used tool $\$^{3}$. As can be seen from the figure, muJava 9 and Proteum 198 are the most frequently-used tools in experimental studies (operate on Java and C, respectively). Other frequently used Java mutation systems are PIT [147, Major [361] and Javalanche [205]. For $\mathrm{C}$, the mutgen framework, used in the study of Andrews et al. [339] and Milu [347] are some of the most frequently-used tools.

Figure 8 depicts the most frequently used languages in experimental studies. It can be seen that mutation is mostly applied at the code/implementation level with the respective test subjects implemented in Java and C. Other commonly-

\footnotetext{
${ }^{2}$ Test prioritisation techniques are typically assessed based on Average Percentage Faults Detected (APFD) or Normalized APFD (NAPFD) 392.

${ }^{3}$ The figure does not include tools that were used in fewer than 4 publications.
} 


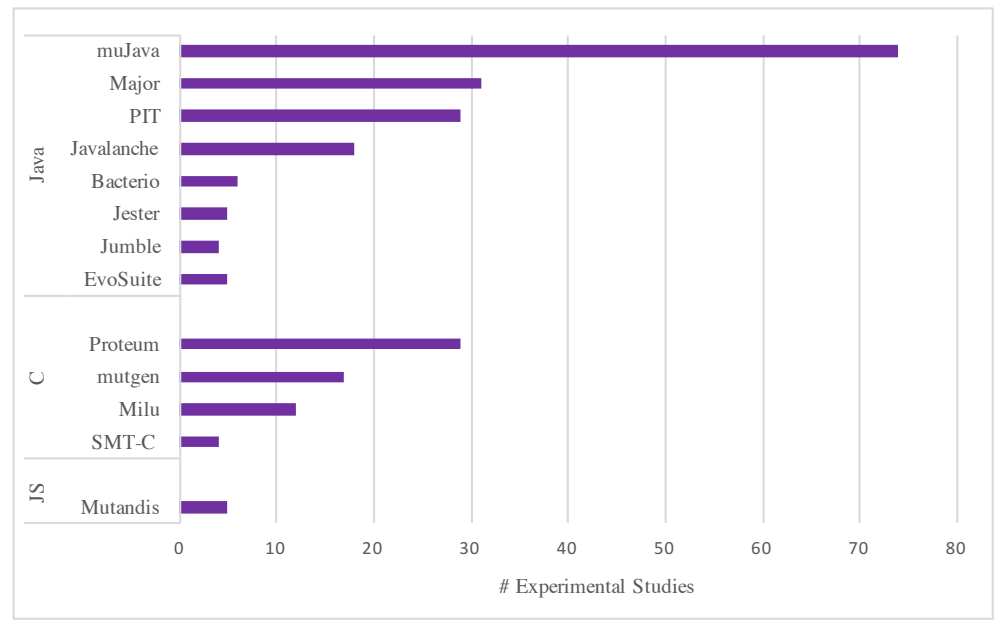

Figure 7: Distribution of mutation testing tools in experimental studies.

used programming languages that are used in mutation experiments include JavaScript and AspectJ. Finally, mutation has also been applied to other test subjects such as security protocols [316], feature models [272] and regular expressions [111, which are distributed in the remaining categories of the figure.

Since mutation testing is increasingly used in experimental studies, potential issues with this practice can have serious implications on many research studies. Although there is some empirical evidence suggesting that mutants behave like real faults [41 43, these are only preliminary results, contradicted by other studies 63, 393, and can be questioned if not suitable experimental care is taken. Unfortunately, recent research has shown that mutation testing is vulnerable to a number of confounding factors, such as those discussed in this section, that researchers should be aware and cater for. The influence of these factors can be severe and lead to questioning many findings of empirical research. In the remainder of this section, we discuss the influential factors that can bias the results of mutation-based test assessment and how we can mitigate them. 


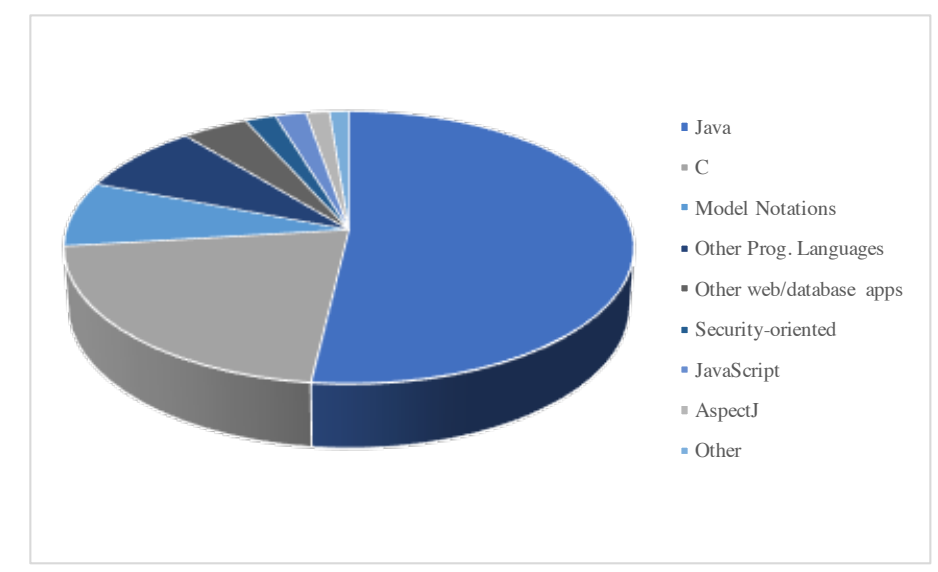

Figure 8: Experimental Studies: Targeted Artefacts.

\subsection{Programming Language and Mutant Operators}

One of the main factors influencing the effectiveness of mutation-based test assessment is the programming language and the mutants that are used in the experimental study [33, 393, 394]. Applying mutation testing requires defining mutants based on the language's constructs. Thus, it is likely that we are not able to use the same mutants for different languages. Additionally, languages following different typing disciplines and programming paradigms may also require different sets of mutant operators. Certain types of mutants might be more effective in one paradigm than another. For instance, strongly typed languages may produce fewer mutants than weakly typed ones [152]. Similarly, object oriented code tends to have simpler method functionality, but more complex interactions among the methods (or classes) than imperative (procedural) languages 395. Therefore, mutants encoding intra-method faults 9 might not be effective. Another example is the Java language runtime checking, which may result in mutants that are easier to kill than those in $\mathrm{C}$.

Namin and Kakarla 393 demonstrated that the correlation between mutants and faults differs significantly across different programming languages. Kintis et al. [152] and Baker and Habli [57] also report significant differences between mutants of different languages, in particular between C - Java and C - Ada, 
respectively. Similarly, there are significant difference across different types of mutant operators, as reported by Namin and Kakarla [393. Kurtz et al. [122] showed that it is hard to select mutant operator sets that perform similarly well on different programs. Therefore, when using mutation testing, it is important to carefully select mutant operators that are appropriate to the programming language studied.

\subsection{Subsumed Mutant Threat}

A major concern when using mutation testing is related to the 'quality' of the employed mutants. In case the mutants we are using are trivial, then we only measure the ability of test suites to cover some parts of the code, instead of their ability to uncover faults at these parts. This problem is called the "subsumed mutant threat" 36. The problem becomes particularly important when we have large number of redundant mutants. Unfortunately, when assessing testing methods, one test technique might achieve a significant advantage over another by killing redundant than non-redundant mutants. This would be a case of inadequately scientific methodology leading to possible incorrect scientific conclusions.

Recent research has shown that redundant mutants tend to skew the mutation score measurement leading to serious threats to the validity of empirical research. Andrews et al. [42] noted that the difficulty of revealing faults and killing mutants may influence the experimental results. Thus, they pointed out that it may be important to filter out the subset of trivial mutants in order to set a representative relation between mutants and real faults. Visser [396] suggested controlling for mutants' reachability in order to identify mutants that are hard to kill (hard to infect and propagate). Papadakis et al. [36] used the notion of mutant subsumption, demonstrating empirically that there is a very good chance (estimated to be more than $60 \%$ for arbitrary experiments) to compromise scientific conclusions, due to this subsumed mutant thread [36]. Similarly, Kurtz et al. 122 replicated previous studies on selective mutation and found that they perform well when considering redundant mutants but perform poorly 
when discarding them.

There are many studies advocating some form of 'refined' mutation score for mitigating the problems caused by mutant redundancies. The first study attempting to address this problem was that of Kintis et al. 39] who suggested using disjoint mutants, i.e., a small representative subset of all mutants in order to remove all the mutant redundancies from the set of mutants that is used for test assessment. Consider that we have a set of $\mathrm{N}$ mutants, a representative subset, say D, means that any test suite that kills this subset of mutants also kills the $\mathrm{N}$ mutants. No redundancy between the mutants of $\mathrm{D}$ means that it is not safe to remove any mutant from this set because in this case we fail to kill all the $\mathrm{N}$ mutants.

Computing the true disjoint mutant set is impossible and thus, in the context of controlled experiments, it is approximated by a test suite. This dynamic approximation of the disjoint mutants can be computed using the Algorithm 1 [36, 167. In this algorithm, the live and duplicate mutants are removed first (from $S$, lines 2 and 3 ). Then, the mutant that is joint (subsuming) with the highest number of live mutants is selected (lines 8 to 15). This is the mutant that it is killed by test cases, which manage to collaterally kill the highest number of other mutants. This mutant is then added to the disjoint set $D$ (line 16) and the joint mutants are removed from $S$ (line 17). This process is repeated until $S$ is empty. Finally, the set of disjoint mutants, $D$, is returned.

Other studies of redundancy reduction include that of Kaminski et al. [76, 163] and Just et al. 164, which suggested removing some instances of the relational and logical mutant operators, in order to improve the accuracy of the mutation score. Ammann et al. 397 introduced the notion of minimal mutants (smallest possible set of mutants) ${ }_{4}^{4}$ and Kurtz et al. [398] suggested selecting the minimal sets of mutants using mutant subsumption graphs. In another

\footnotetext{
${ }^{4}$ The difference between the notions of minimal and disjoint mutants is that minimal mutants is the smallest possible representative set of mutants while the disjoint ones is a set that has no redundancies (perhaps not the minimal one) 33 39].
} 
study, Kurtz et al. [169] proposed using symbolic execution to approximate subsuming mutants. Papadakis et al. [37] and Kintis et al. [152] suggested using compiler optimisations to remove duplicated mutants (a special form of redundant mutants) as a way to strengthen experimental rigour.

Overall, all these studies found that a large percentage of mutants are redundant, indicating potential inflation problems for the studies that have not take account of redundancy. Kintis et al. 39 reports that disjoint mutants were approximately $9 \%$ of all the mutants, Ammann et al. 397. that minimal mutants were $1.2 \%$ and Kurtz et al. 398 that they were 4\%. These results motivated the work of Papadakis et al. 36 that found that redundant mutants can bias experimental results (approximately $60 \%$ for arbitrary experiments). Therefore, researchers should identify and discard as many subsumed mutants as possible before conducting any test assessment.

\subsection{Test Suite Strength and Size}

Failure to account for test suite strength can also adversely affect the scientific findings of empirical studies. Chekam et al. [6] studied the relation between faults and mutants, reports that low strength test suites are vulnerable to noise effects "two studies with below- threshold coverage may yield different findings, even when the experimenters follow identical experimental procedures.". Thus, their study concluded that test suite strength plays a central role when conducting an experiment.

In particular, the study of Chekam et al. [6] showed that there is no practical difference between test criteria when relatively low-strength test suites are used. By contradiction, higher-strength test suites yield larger differences for test criteria. This is particularly important, because it indicates that empirical studies need to improve the strength of their test suites before conducting the experiment. Unfortunately, the mutation strength (over other test techniques) is only observable using strong test suites. For instance, one might conclude that a test technique or criterion is ineffective (compared to another), while in fact it is not, simply because the superiority of this criterion is only observable 


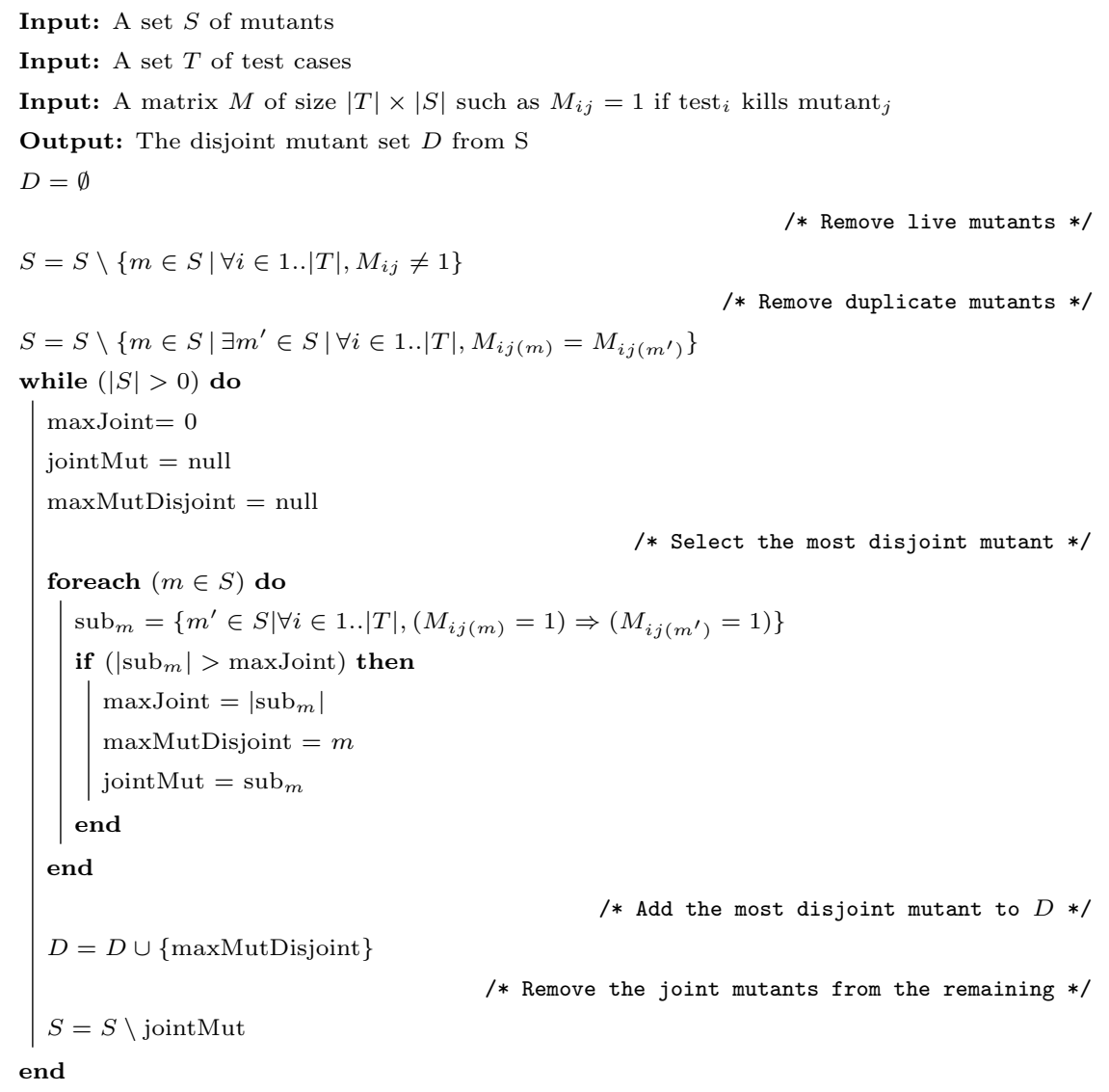

using stronger test suites.

Recent studies have also identified that test suite size (number of test cases) introduces another confounding factor that should be brought under experimental control [61. Going a step further, Namin and Kakarla 393] observed that by using different test suite sizes, an experimenter will observe different correlations between faults and mutants. Since test suite size can be considered as a proxy measure of test suite's strength, this finding re-confirms the findings of Chekam et al. [6], suggesting experiments should consider both test suite size and test suite strength.

Another source of variation of empirical results is due to selection of can- 
didate test cases. Consider the case where we want to compare two test techniques. In this case, we need two test suites, one that simulates the result of the first technique and one that simulates the result of the second technique. This is usually performed by randomly sampling of test cases from a test pool or by randomly generating test suites. Thus, two sets of test cases are to be compared. A problem typically arrises in this scenario is that these two test suites need to adequately simulate the results of the techniques. Therefore, the two sets need to be free from redundant test cases [33, 167, 397, which might otherwise inadvertently bias experimental results. An easy way to ameliorate this problem is to select test cases that only increase coverage, while discarding all the others 33,42 .

Another concern derives from the test case selection. As this may be stochastic, it is likely that different selection of test cases (at random) may result in different results; perhaps very different results if the experimenter happens to be unlucky. To reduce this problem, researchers usually select multiple sets of test cases and perform an inferential statistical analysis on the set of results as a whole, 399, 400]. Delamaro and Offutt [401] investigated the influence of using multiple sets of test cases (selected randomly) and found that "averaging over multiple programs was effective in reducing the variance in the mutation scores introduced by specific tests". Therefore, they found that in case it is too expensive to perform multiple repeated experiments a single test set (per program) over a relatively large number of subject can be enough to provide accurate average values.

Overall, researchers are advised to carefully select their test suites. Depending on the evaluation scenario it might be important to control for test suite size and reduce redundant test cases.

\subsection{Mutation Testing Tools}

An often-ignored parameter that can also inadvertently bias experimental findings relates to the choice of mutation testing tool. Mutation testing tools implement different operators and have different implementation details, most 
of which can influence the experimental outcome [394]. As already explained, the choice of mutant operators affects significantly the results of an experiment. However, different implementations of the same operators are likely to produce different mutants and merely provide divergent results.

The studies of Kintis et al. [394, 402] and Gopinath et al. [403] demonstrate that there is a large degree of disagreement between the judgements made by the most popular Java mutation testing tools. The studies of Kintis et al. [394] and Marki and Lindstrm [404] cross evaluate the Java mutation testing tools and identify specific implementation weaknesses. This motivated the work of Laurent et al. [33, 167, who compared Java mutation testing tools and implemented one (called PIT $_{\mathrm{RV}}$ [147]) that is "at least as strong as the mutants of all the other tools together". Unfortunately, these studies are only concerned with Java so there is no clear evidence concerning the C mutation testing tools (or tools for other less widely-studied languages). Taken together, these results suggest that the choice of a mutation tool need to be carefully introduced and justified in best practice empirical studies.

\subsection{Clean Program Assumption}

Mutation-based test assessment can be viewed as a simulation that involves two 'roles'; the faults role (played by the mutants) and the 'oracle' role (played by the original program). By aligning this simulation to the reality, we can say that developers produce the faulty programs (simulated by the mutants) which they test using a test oracle (simulated by the original program). Naturally, testers apply their tools and techniques on the mutant program versions, check whether they can find any unexpected behaviour, as defined by the test oracle and report on any bugs found.

As intuitive as this seems, the practice of test assessment is performed differently. It is a common practice to apply tools and test techniques on the original program and then check their fault-revealing power by executing tests on the mutants. This practice may be less time-consuming but it makes an implicit assumption that coverage measurements (or the application of test techniques) on 
the original program are representative (or very similar) of those on the mutant programs. This assumption is called the "Clean Program Assumption" (CPA) [6. The assumption can be problematic since test suites are assessed on the mutant program versions instead of the original program from which (and for which) they were applied.

Unfortunately, Chekam et al. [6] demonstrated that the CPA does not hold and therefore cannot be relied upon. The study also showed that CPA has the potential of changing the outcome of empirical studies if not brought under experimental control. Overall, the Chekam et al. revisited previous empirical questions concerning the usefulness of test adequacy criteria, using a robust methodology that accounts for CPA and showed that mutation testing outperform statement and branch coverage for real fault revelation. These results suggest that experiments dealing with the real fault revelation question, should report on the CPA. If it is not possible to take CPA into account (potentially due to execution cost), researchers are advised to report the amount of time required by the performed study.

\section{A Seven-Point Check List of Best Practices on using Mutation Testing in Controlled Experiments}

The fundamental experimental factors surveyed in Section 9 highlight the many pitfalls that can compromise or even invalidate the scientific findings and conclusions of a controlled experiment that uses mutation testing. It can be a daunting challenge for experimenters and researchers to be sure they have catered for all of the potential threats to validity that have accrued over four decades of literature recording the development of mutation testing.

Therefore, to address this challenge, in this section, we provide a simple seven-step checklist that aims to give experimenters the confidence that they are compliant with best practice reporting of results. Ensuring that all seven steps are met is relatively straightforward, because it simply involves explaining and justifying choices that may affect conclusion validity. Nevertheless, experi- 
menters who follow these seven steps help other researchers replicate and investigate, properly, the influence of such potentially confounding factors, thereby contributing to the overall experimental robustness of their study.

1. Mutant Selection: Explain the choice of mutant operators. One of the most important things that experimenters need to explain is the appropriateness of the chosen mutant operators with respect to the programming language used.

2. Mutation Testing Tool: Justify the choice of mutation testing tool. The choice of mutation testing tool needs to be made carefully as at the current state, mutation testing tools differ significantly [394, 402]. To support the reproducibility and comprehension of the experimental results, researchers should also clearly describe the exact version of the employed mutation testing tool. If the used tool is not a publicly available, researchers should list the exact transformation rules (mutant instances supported by each operator [394, 402]) that are supported by the mutant operators selected. Unfortunately, our survey found that more than a quarter of the empirical studies does not report such details. The objective is to provide readers with the low-level details that might vary from one study to another, so that these can be accounted for in subsequent studies.

3. Mutant redundancy: Justify the steps taken to control mutant redundancy. As we discussed in Section 9.3, mutant redundancy may have a large impact on the validity of the assessment. Therefore, it is important to explain how mutant redundancy is handled (perhaps in the threats to validity section). Where possible, experimenters are advised to additionally use techniques like TCE [152] to remove the duplicate mutants (in case the interest is on the achieved score of a technique), or a dynamic approximation of the disjoint mutation score [33, 36] (in case the interest is on comparing test techniques). As already discussed, the approximation of the disjoint mutants can be made by using Algorithm 1. In case these techniques are expensive, researchers are advised to clarify this and con- 
trast their findings on a (small) sample of cases where mutant redundancy is controlled.

4. Test suite choice and size: Explain the choice of test suite and any steps taken to account for the effects of test suite size, where appropriate. Ideally, an experimenter would like to have large, diverse (i.e., mutants are killed by multiple test cases) and high-strength (i.e., killing the majority of the mutants) test suites. As such test suites are rare in most of the open-source projects, researchers are advised to demonstrate and contrast their findings with a (small) sample of subjects with strong and diverse test suites (perhaps in addition to the chosen subjects). Alternatively, experimenters may consider using automated tools to augment their test suites. Overall, the objective is to allow other researchers to create a similar test suite and/or to experiment with different choice of suite and measure the effects of such choices.

5. Clean Program Assumption: Explain whet the study relies on the CPA assumption. Ideally, where possible, the CPA should not be relied upon; testing should be applied to the faulty programs (instead of the clean, non-faulty ones). If this is not possible (potentially due to execution cost or lack of resources), researchers are advised to note the reliance on the CPA. Its effects may be small in some cases, justifying reliance on this assumption. Either way, explicitly stating whether or not it is relied upon will aid clarity and facilitate subsequent studies.

6. Multiple experimental repetitions: Clarify the number of experimental repetitions. Ideally, when techniques make stochastic choices they should be assessed by multiple experimental repetitions [400, 405]. In practice, this might not be possible due to the required execution time or other constraints. In this case, researchers have to choose between experiments with many subjects but few repetitions or experiments with few subjects and many repetitions; research suggests that it is preferable to choose the second option 401. Of course, this choice needs to be clarified according to the specific context and goals of the study. 
7. Presentation of the results: Clarify the granularity level of the empirical results. Many empirical studies compute mutation scores over the whole subject projects they are using (one score per project). Since, this practice may not generalise to other granularity level 5 (such as unit level) [167, researchers should report and explain the suitability of the chosen granularity level at the given application context.

\section{Conclusion and Future Directions}

This chapter surveyed the recent trends and advances on Mutation Testing. It offers a concise description of the mutation testing problems, methods, applications and best practices for applying mutation testing (either as a test technique or as an experimental methodology). Based on the data we collected, we demonstrate that there is a growing interest in the subject. Interestingly, even eight years after the first observation of this trend, by Jia and Harman [27, the interest in the field is still increasing markedly.

The interest in the field is related to both fundamental research advances and practical applications such as tool support and use in controlled experiments. Our analysis shows that many tools and techniques have been introduced these last 10 years. Many of these advances are already widely used by researchers. At the same time major companies report that they experiment with mutation in order to include in their practices. Hopefully, practitioners will soon use mutation as well. All these observations may be seen as evidence supporting the claim that mutation testing is reaching a state of maturity. In summary, the research interest in mutation testing is divided into the following general categories:

- Solutions to the problems of mutation analysis (fundamental advances of mutation).

\footnotetext{
${ }^{5}$ Two methods can have a similar number of mutants killed on a project (overall number), but quite different numbers, of mutants killed, on the individual units of the project.
} 
- Mutation applied on new languages and artefacts. New mutation testing tools also appear.

- Use of mutants as a means to support other software engineering activities (e.g., fault localisation [17]).

- Use of mutation testing advances to support controlled experiments.

Recent work in the area focuses on building scalable and practical tools that can push mutation testing towards industry and everyday use. The rest of this section is dedicated to summarise the mutation testing open problems, barriers and areas that we believe will attract attention in the near future.

\subsection{Open Problems}

One of the main open problems of mutation regards the detection of the equivalent and redundant mutants. As we already discussed, there are many techniques tackling this problem, either directly or indirectly, but unfortunately the problem remains largely unresolved. Overall, the current research results show that only few of the mutants produced (approximately 5\%) is practically useful. The rest is noise to the process with severe consequences [36].

Overall, mutation testing requires models that will guide the mutations towards small semantic deviations that are in a sense disjoint, instead of blind syntactical mutations. Unfortunately, there is no clear theory or consensus on which types and instances of mutants we should use. Some initial results indicate that almost all the mutant operators are of some value. The fact that most of the existing tools are limited to a small number of mutant operators is restrictive and to some extend arbitrary. Thus, in future, mutation may be tailored towards few diverse and 'useful' mutants that bring value to the tester (regardless of the operators used) [41].

The lack of clear theory on which mutants are of some value has restricted most of the previous research on first order mutation. Higher order mutation appears to have similar characteristics with the first order mutation as it produces subtle mutants. Of course the great majority of them are redundant, but 
in theory a smart mutant selection process can identify them. Therefore, future mutation may identify ways to generate and use those valuable higher order mutants.

Another important aspect concerns the automatic mutation-based generation of test cases and test oracles. Although the last ten years there are major advances on this area of research, the problem remains. Most of the automated approaches fail to kill a substantial number of mutants and recent empirical evaluations show that automatic test generation techniques fail to cover most of the critical program areas. Therefore, there is little work on improving test suites using mutants. Perhaps this is attributed to the lack of understanding and modelling of the error propagation. Recent research has shown that failed mutant propagation is the basic ingredient that makes mutation testing powerful [6]. Much work remains to be done until we can automatically produce high quality test cases through high quality mutants.

Although researchers have identified mutation as a strong test criterion, there is neither clear understanding nor much empirical evidence concerning whether and when mutants are correlated with real faults. What types of faults are not captured by simple or complex mutants? What percentage of future regression errors can we capture with mutations? When is it appropriate to stop the testing process? How should we integrate mutation testing into our development process? Of course these questions need to be answered under the light of specific development paradigms and application domains. These are open questions, hopefully to be answered by future research.

Model-based mutation is one of the areas that has not been researched much (compared to code-based mutation) over the last years. Despite this, we see a growing interest towards this direction. There is a recent dedicated survey on this subject [30] and multiple high profile publications over the last couple of years. Additionally, very recently efficient and scalable tools have been built, e.g., the VIBeS tool [12, which hopefully will push the research in this area further.

Finally, there are many new areas of research that can benefit from the use 
of mutants. The current trend is to explore the behaviour space of mutants, instead of the original program, to identify several interesting aspects, either functional or non-functional. Thus, the conformance of models, the generation and improvement of models, the improvements of program security and debugging activities are only a few examples where mutants have been shown to be spectacularly useful and effective. Future research is heading towards this line of research with many new and exciting applications of mutation analysis.

\section{References}

[1] J. Offutt, A mutation carol: Past, present and future, Information \& Software Technology 53 (10) (2011) 1098-1107. doi:10.1016/j.infsof. 2011.03 .007

URL https://doi.org/10.1016/j.infsof .2011.03.007

[2] P. E. Black, V. Okun, Y. Yesha, Mutation operators for specifications, in: The Fifteenth IEEE International Conference on Automated Software Engineering, ASE 2000, Grenoble, France, September 11-15, 2000, 2000, p. 81. doi:10.1109/ASE.2000.873653 URL https://doi.org/10.1109/ASE.2000.873653

[3] X. Devroey, G. Perrouin, M. Papadakis, A. Legay, P. Schobbens, P. Heymans, Automata language equivalence vs. simulations for model-based mutant equivalence: An empirical evaluation, in: 2017 IEEE International Conference on Software Testing, Verification and Validation, ICST 2017, Tokyo, Japan, March 13-17, 2017, 2017, pp. 424-429. doi: 10.1109/ICST.2017.46.

URL https://doi.org/10.1109/ICST . 2017.46

[4] M. E. Delamaro, J. C. Maldonado, A. P. Mathur, Interface mutation: An approach for integration testing, IEEE Trans. Software Eng. 27 (3) (2001) 228-247. doi:10.1109/32.910859.

URL https://doi .org/10.1109/32.910859 
[5] P. R. Mateo, M. P. Usaola, J. Offutt, Mutation at system and functional levels, in: Third International Conference on Software Testing, Verification and Validation, ICST 2010, Paris, France, April 7-9, 2010, Workshops Proceedings, 2010, pp. 110-119. doi:10.1109/ICSTW.2010.18.

URL https://doi .org/10.1109/ICSTW .2010.18

[6] T. T. Chekam, M. Papadakis, Y. L. Traon, M. Harman, An empirical study on mutation, statement and branch coverage fault revelation that avoids the unreliable clean program assumption, in: Proceedings of the 39th International Conference on Software Engineering, ICSE 2017, Buenos Aires, Argentina, May 20-28, 2017, 2017, pp. 597-608.

URL http://dl . acm . org/citation . cfm?id=3097440

[7] P. Delgado-Pérez, I. Medina-Bulo, F. Palomo-Lozano, A. GarcíaDomínguez, J. J. Domínguez-Jiménez, Assessment of class mutation operators for $\mathrm{C}++$ with the mucpp mutation system, Information \& Software Technology 81 (2017) 169-184. doi:10.1016/j.infsof.2016.07.002.

URL https://doi .org/10.1016/j .inf sof .2016.07.002

[8] A. Derezinska, K. Kowalski, Object-oriented mutation applied in common intermediate language programs originated from c\#, in: Fourth IEEE International Conference on Software Testing, Verification and Validation, ICST 2012, Berlin, Germany, 21-25 March, 2011, Workshop Proceedings, 2011, pp. 342-350. doi:10.1109/ICSTW.2011.54. URL https://doi .org/10.1109/ICSTW.2011.54

[9] Y. Ma, J. Offutt, Y. R. Kwon, Mujava: an automated class mutation system, Softw. Test., Verif. Reliab. 15 (2) (2005) 97-133. doi:10.1002/ stvr.308.

URL https://doi.org/10.1002/stvr.308

[10] S. Mirshokraie, A. Mesbah, K. Pattabiraman, Efficient javascript mutation testing, in: Sixth IEEE International Conference on Software Testing, Verification and Validation, ICST 2013, Luxembourg, Luxembourg, 
March 18-22, 2013, 2013, pp. 74-83. doi:10.1109/ICST.2013.23.

URL http://dx.doi.org/10.1109/ICST .2013.23

[11] N. Li, M. West, A. Escalona, V. H. S. Durelli, Mutation testing in practice using ruby, in: Eighth IEEE International Conference on Software Testing, Verification and Validation, ICST 2015 Workshops, Graz, Austria, April 13-17, 2015, 2015, pp. 1-6. doi:10.1109/ICSTW.2015.7107453.

URL https://doi.org/10.1109/ICSTW.2015.7107453

[12] X. Devroey, G. Perrouin, M. Papadakis, A. Legay, P. Schobbens, P. Heymans, Featured model-based mutation analysis, in: Proceedings of the 38th International Conference on Software Engineering, ICSE 2016, Austin, TX, USA, May 14-22, 2016, 2016, pp. 655-666. doi:10.1145/ 2884781.2884821 .

URL http://doi .acm.org/10.1145/2884781.2884821

[13] Y. Ma, Y. R. Kwon, J. Offutt, Inter-class mutation operators for java, in: 13th International Symposium on Software Reliability Engineering (ISSRE 2002), 12-15 November 2002, Annapolis, MD, USA, 2002, pp. 352-366. doi:10.1109/ISSRE. 2002.1173287.

URL https://doi.org/10.1109/ISSRE.2002.1173287

[14] D. Le, M. A. Alipour, R. Gopinath, A. Groce, Mucheck: an extensible tool for mutation testing of haskell programs, in: International Symposium on Software Testing and Analysis, ISSTA '14, San Jose, CA, USA - July 21 - 26, 2014, 2014, pp. 429-432. doi:10.1145/2610384.2628052.

URL http://doi .acm.org/10.1145/2610384.2628052

[15] E. Omar, S. Ghosh, An exploratory study of higher order mutation testing in aspect-oriented programming, in: 23rd IEEE International Symposium on Software Reliability Engineering, ISSRE 2012, Dallas, TX, USA, November 27-30, 2012, 2012, pp. 1-10. doi:10.1109/ISSRE.2012.6.

URL https://doi.org/10.1109/ISSRE.2012.6 
[16] J. Tuya, M. J. S. Cabal, C. de la Riva, Mutating database queries, Information \& Software Technology 49 (4) (2007) 398-417. doi:10.1016/j. infsof.2006.06.009.

URL https://doi.org/10.1016/j.infsof .2006.06.009

[17] M. Papadakis, Y. L. Traon, Metallaxis-fl: mutation-based fault localization, Softw. Test., Verif. Reliab. 25 (5-7) (2015) 605-628. doi:10.1002/ stvr.1509. URL http://dx.doi.org/10.1002/stvr.1509

[18] C. Le Goues, T. Nguyen, S. Forrest, W. Weimer, Genprog: A generic method for automatic software repair, IEEE Trans. Software Eng. 38 (1) (2012) 54-72. doi:10.1109/TSE.2011.104 URL https://doi.org/10.1109/TSE.2011.104

[19] T. Loise, X. Devroey, G. Perrouin, M. Papadakis, P. Heymans, Towards security-aware mutation testing, in: 2017 IEEE International Conference on Software Testing, Verification and Validation Workshops, ICST Workshops 2017, Tokyo, Japan, March 13-17, 2017, 2017, pp. 97-102. doi:10.1109/ICSTW.2017.24.

URL http://dx.doi.org/10.1109/ICSTW.2017.24

[20] Y. Jia, F. Wu, M. Harman, J. Krinke, Genetic improvement using higher order mutation in: Genetic and Evolutionary Computation Conference, GECCO 2015, Madrid, Spain, July 11-15, 2015, Companion Material Proceedings, 2015, pp. 803-804. doi:10.1145/2739482.2768417. URL http://doi .acm.org/10.1145/2739482.2768417

[21] W. B. Langdon, B. Y. H. Lam, M. Modat, J. Petke, M. Harman, Genetic improvement of GPU software, Genetic Programming and Evolvable Machines 18 (1) (2017) 5-44. doi:10.1007/s10710-016-9273-9.

URL https ://doi .org/10.1007/s10710-016-9273-9

[22] R. G. Hamlet, Testing Programs with the Aid of a Compiler, IEEE Transactions on Software Engineering 3 (4) (1977) 279-290. 
[23] R. A. DeMillo, R. J. Lipton, F. G. Sayward, Program Mutation: A New Approach to Program Testing, in: Infotech State of the Art Report, Software Testing, Vol. 2, 1979, pp. 107-126.

[24] R. A. DeMillo, R. J. Lipton, F. G. Sayward, Hints on test data selection: Help for the practicing programmer, IEEE Computer 11 (4) (1978) 34-41. doi:10.1109/C-M.1978.218136.

URL https://doi.org/10.1109/C-M.1978.218136

[25] R. A. DeMillo, Test adequacy and program mutation, in: Proceedings of the 11th International Conference on Software Engineering, Pittsburg, PA, USA, May 15-18, 1989., 1989, pp. 355-356. doi:10.1145/74587.74634. URL http://doi .acm.org/10.1145/74587.74634

[26] A. J. Offutt, R. Untch, Mutation 2000: Uniting the orthogonal, in: W. E. Wong (Ed.), Mutation 2000, Kluwer, San Jose, California, USA, 2001, pp. $45-55$.

[27] Y. Jia, M. Harman, An analysis and survey of the development of mutation testing, IEEE Trans. Software Eng. 37 (5) (2011) 649-678. doi:10.1109/ TSE. 2010.62

URL http://dx.doi.org/10.1109/TSE.2010.62

[28] L. Madeyski, W. Orzeszyna, R. Torkar, M. Jozala, Overcoming the equivalent mutant problem: A systematic literature review and a comparative experiment of second order mutation, IEEE Trans. Software Eng. 40 (1) (2014) 23-42. doi:10.1109/TSE.2013.44

URL https://doi.org/10.1109/TSE.2013.44

[29] F. C. Souza, M. Papadakis, V. H. S. Durelli, M. E. Delamaro, Test data generation techniques for mutation testing: A systematic mapping, in: Proceedings of the 11th ESELAW, 2014, pp. 1-14.

[30] F. Belli, C. J. Budnik, A. Hollmann, T. Tuglular, W. E. Wong, Modelbased mutation testing - approach and case studies, Sci. Comput. Pro- 
gram. 120 (2016) 25-48. doi:10.1016/j.scico.2016.01.003

URL https://doi.org/10.1016/j.scico.2016.01.003

[31] R. A. Silva, S. do Rocio Senger de Souza, P. S. L. de Souza, A systematic review on search based mutation testing, Information \& Software Technology 81 (2017) 19-35. doi:10.1016/j.infsof .2016.01.017

URL https://doi.org/10.1016/j.inf sof .2016.01.017

[32] P. Ammann, J. Offutt, Introduction to software testing, 2nd Edition, Cambridge University Press, 2016.

[33] T. Laurent, M. Papadakis, M. Kintis, C. Henard, Y. L. Traon, A. Ventresque, Assessing and improving the mutation testing practice of pit, in: 2017 IEEE International Conference on Software Testing, Verification and Validation (ICST), 2017, pp. 430-435. doi:10.1109/ICST.2017.47.

[34] A. J. Offutt, A. Lee, G. Rothermel, R. H. Untch, C. Zapf, An experimental determination of sufficient mutant operators, ACM Trans. Softw. Eng. Methodol. 5 (2) (1996) 99-118. doi:10.1145/227607.227610.

URL http://doi .acm .org/10.1145/227607.227610

[35] J. B. Goodenough, S. L. Gerhart, Toward a theory of test data selection, IEEE Trans. Software Eng. 1 (2) (1975) 156-173. doi:10.1109/TSE. 1975.6312836 .

URL https : //doi.org/10.1109/TSE.1975.6312836

[36] M. Papadakis, C. Henard, M. Harman, Y. Jia, Y. L. Traon, Threats to the validity of mutation-based test assessment, in: Proceedings of the 25th International Symposium on Software Testing and Analysis, ISSTA 2016, Saarbrücken, Germany, July 18-20, 2016, 2016, pp. 354-365. doi: $10.1145 / 2931037.2931040$

URL http://doi .acm .org/10.1145/2931037.2931040

[37] M. Papadakis, Y. Jia, M. Harman, Y. L. Traon, Trivial compiler equivalence: A large scale empirical study of a simple, fast and effective equiva- 
lent mutant detection technique, in: 37th IEEE/ACM International Conference on Software Engineering, ICSE 2015, Florence, Italy, May 16-24, 2015, Volume 1, 2015, pp. 936-946. doi:10.1109/ICSE.2015.103

URL http://dx.doi.org/10.1109/ICSE.2015.103

[38] Y. Jia, M. Harman, Higher order mutation testing, Information \& Software Technology 51 (10) (2009) 1379-1393. doi:10.1016/j.infsof. 2009.04 .016

URL https://doi.org/10.1016/j.inf sof .2009.04.016

[39] M. Kintis, M. Papadakis, N. Malevris, Evaluating mutation testing alternatives: A collateral experiment, in: 17th Asia Pacific Software Engineering Conference, APSEC 2010, Sydney, Australia, November 30 December 3, 2010, 2010, pp. 300-309. doi:10.1109/APSEC.2010.42. URL https://doi .org/10.1109/APSEC .2010.42

[40] Karl popper, https://en.wikiquote.org/wiki/Karl_Popper, accessed: 2017-06-10.

[41] M. Papadakis, D. Shin, S. Yoo, D. Bae, Are mutation scores correlated with real fault detection? a large scale empirical study on the relationship between mutants and real faults, in: Proceedings of the 40th International Conference on Software Engineering, ICSE 2018, Gothenburg, Sweden, May 27 - 3 June, 2018, 2018.

[42] J. H. Andrews, L. C. Briand, Y. Labiche, A. S. Namin, Using mutation analysis for assessing and comparing testing coverage criteria, IEEE Trans. Software Eng. 32 (8) (2006) 608-624. doi:10.1109/TSE.2006.83.

URL https://doi.org/10.1109/TSE.2006.83

[43] R. Just, D. Jalali, L. Inozemtseva, M. D. Ernst, R. Holmes, G. Fraser, Are mutants a valid substitute for real faults in software testing?, in: Proceedings of the 22nd ACM SIGSOFT International Symposium on Foundations of Software Engineering, (FSE-22), Hong Kong, China, November 16 - 22, 
2014, 2014, pp. 654-665. doi:10.1145/2635868.2635929

URL http://doi.acm.org/10.1145/2635868.2635929

[44] P. G. Frankl, S. N. Weiss, An experimental comparison of the effectiveness of the all-uses and all-edges adequacy criteria, in: Symposium on Testing, Analysis, and Verification, 1991, pp. 154-164. doi:10.1145/120807. 120821 .

URL http://doi.acm.org/10.1145/120807.120821

[45] P. G. Frankl, S. N. Weiss, An experimental comparison of the effectiveness of branch testing and data flow testing, IEEE Trans. Software Eng. 19 (8) (1993) 774-787. doi:10.1109/32.238581.

URL http://dx.doi.org/10.1109/32.238581

[46] A. J. Offutt, J. Pan, K. Tewary, T. Zhang, An experimental evaluation of data flow and mutation testing Softw., Pract. Exper. 26 (2) (1996) 165-176. doi:10.1002/(SICI) 1097-024X(199602) 26:2<165: : AID-SPE5>3.0.CO;2-K.

URL http://dx.doi.org/10.1002/(SICI)1097-024X(199602)26: 2<165: :AID-SPE5>3.0.CO;2-K

[47] P. G. Frankl, S. N. Weiss, C. Hu, All-uses vs mutation testing: An experimental comparison of effectiveness, Journal of Systems and Software 38 (3) (1997) 235-253. doi:10.1016/S0164-1212(96)00154-9.

URL http://dx.doi .org/10.1016/S0164-1212(96)00154-9

[48] P. G. Frankl, O. Iakounenko, Further empirical studies of test effectiveness, in: SIGSOFT '98, Proceedings of the ACM SIGSOFT International Symposium on Foundations of Software Engineering, Lake Buena Vista, Florida, USA, November 3-5, 1998, 1998, pp. 153-162. doi:10.1145/288195.288298,

URL http://doi .acm.org/10.1145/288195.288298

[49] L. C. Briand, D. Pfahl, Using simulation for assessing the real impact of test-coverage on defect-coverage, IEEE Trans. Reliability 49 (1) (2000) 
60-70. doi:10.1109/24.855537.

URL http://dx .doi .org/10.1109/24.855537

[50] M. Chen, M. R. Lyu, W. E. Wong, Effect of code coverage on software reliability measurement, IEEE Trans. Reliability 50 (2) (2001) 165-170. doi:10.1109/24.963124.

URL http://dx.doi .org/10.1109/24.963124

[51] A. S. Namin, J. H. Andrews, The influence of size and coverage on test suite effectiveness, in: Proceedings of the Eighteenth International Symposium on Software Testing and Analysis, ISSTA 2009, Chicago, IL, USA, July 19-23, 2009, 2009, pp. 57-68. doi:10.1145/1572272.1572280.

URL http://doi .acm.org/10.1145/1572272.1572280

[52] N. Li, U. Praphamontripong, J. Offutt, An experimental comparison of four unit test criteria: Mutation, edge-pair, all-uses and prime path coverage, in: Second International Conference on Software Testing Verification and Validation, ICST 2009, Denver, Colorado, USA, April 1-4, 2009, Workshops Proceedings, 2009, pp. 220-229. doi:10.1109/ICSTW.2009. 30.

URL http://dx .doi .org/10.1109/ICSTW .2009.30

[53] M. Papadakis, N. Malevris, An empirical evaluation of the first and second order mutation testing strategies, in: Third International Conference on Software Testing, Verification and Validation, ICST 2010, Paris, France, April 7-9, 2010, Workshops Proceedings, 2010, pp. 90-99. doi:10.1109/ ICSTW.2010.50.

URL http://dx.doi.org/10.1109/ICSTW.2010.50

[54] I. Ciupa, A. Pretschner, M. Oriol, A. Leitner, B. Meyer, On the number and nature of faults found by random testing, Softw. Test., Verif. Reliab. 21 (1) (2011) 3-28. doi:10.1002/stvr.415. URL http://dx.doi .org/10.1002/stvr.415 
[55] S. Kakarla, S. Momotaz, A. S. Namin, An evaluation of mutation and data-flow testing: A meta-analysis, in: Fourth IEEE International Conference on Software Testing, Verification and Validation, ICST 2012, Berlin, Germany, 21-25 March, 2011, Workshop Proceedings, 2011, pp. 366-375. doi:10.1109/ICSTW.2011.51.

URL http://dx.doi.org/10.1109/ICSTW.2011.51

[56] Y. Wei, B. Meyer, M. Oriol, Is Branch Coverage a Good Measure of Testing Effectiveness?, Springer Berlin Heidelberg, Berlin, Heidelberg, 2012, pp. 194-212. doi:10.1007/978-3-642-25231-0_5.

[57] R. Baker, I. Habli, An empirical evaluation of mutation testing for improving the test quality of safety-critical software, IEEE Trans. Software Eng. 39 (6) (2013) 787-805. doi:10.1109/TSE.2012.56.

URL https://doi.org/10.1109/TSE.2012.56

[58] M. M. Hassan, J. H. Andrews, Comparing multi-point stride coverage and dataflow coverage, in: 35th International Conference on Software Engineering, ICSE '13, San Francisco, CA, USA, May 18-26, 2013, 2013, pp. 172-181. doi:10.1109/ICSE.2013.6606563.

URL http://dx.doi.org/10.1109/ICSE.2013.6606563

[59] M. Gligoric, A. Groce, C. Zhang, R. Sharma, M. A. Alipour, D. Marinov, Comparing non-adequate test suites using coverage criteria, in: International Symposium on Software Testing and Analysis, ISSTA '13, Lugano, Switzerland, July 15-20, 2013, 2013, pp. 302-313. doi:10.1145/2483760. 2483769 .

URL http://doi.acm.org/10.1145/2483760.2483769

[60] M. Gligoric, A. Groce, C. Zhang, R. Sharma, M. A. Alipour, D. Marinov, Guidelines for coverage-based comparisons of non-adequate test suites, ACM Trans. Softw. Eng. Methodol. 24 (4) (2015) 22:1-22:33. doi:10. $1145 / 2660767$.

URL http://doi.acm.org/10.1145/2660767 
[61] L. Inozemtseva, R. Holmes, Coverage is not strongly correlated with test suite effectiveness, in: 36th International Conference on Software Engineering, ICSE '14, Hyderabad, India - May 31 - June 07, 2014, 2014, pp. 435-445. doi:10.1145/2568225.2568271.

URL http://doi .acm.org/10.1145/2568225.2568271

[62] R. Gopinath, C. Jensen, A. Groce, Code coverage for suite evaluation by developers, in: 36th International Conference on Software Engineering, ICSE '14, Hyderabad, India - May 31 - June 07, 2014, 2014, pp. 72-82. doi:10.1145/2568225.2568278. URL http://doi . acm.org/10.1145/2568225.2568278

[63] I. Ahmed, R. Gopinath, C. Brindescu, A. Groce, C. Jensen, Can testedness be effectively measured?, in: Proceedings of the 24th ACM SIGSOFT International Symposium on Foundations of Software Engineering, FSE 2016, Seattle, WA, USA, November 13-18, 2016, 2016, pp. 547-558. doi : $10.1145 / 2950290.2950324$

URL http://doi .acm.org/10.1145/2950290.2950324

[64] R. Ramler, T. Wetzlmaier, C. Klammer, An empirical study on the application of mutation testing for a safety-critical industrial software system in: Proceedings of the Symposium on Applied Computing, SAC 2017, Marrakech, Morocco, April 3-7, 2017, 2017, pp. 1401-1408. doi: $10.1145 / 3019612.3019830$

URL http://doi.acm.org/10.1145/3019612.3019830

[65] D. Binkley, S. Danicic, T. Gyimóthy, M. Harman, A. Kiss, L. Ouarbya, Formalizing executable dynamic and forward slicing, in: $4^{\text {th }}$ International Workshop on Source Code Analysis and Manipulation (SCAM 04), Los Alamitos, California, USA, 2004, pp. 43-52.

[66] K. H. Brodersen, F. Gallusser, J. Koehler, N. Remy, S. L. Scott, Inferring causal impact using bayesian structural time-series models, Annals of Applied Statistics 9 (2015) 247-274. 
[67] L. J. Morell, A theory of fault-based testing, IEEE Trans. Software Eng. 16 (8) (1990) 844-857. doi:10.1109/32.57623

URL http://dx.doi.org/10.1109/32.57623

[68] A. J. Offutt, Investigations of the software testing coupling effect, ACM Trans. Softw. Eng. Methodol. 1 (1) (1992) 5-20. doi:10.1145/125489. 125473 .

URL http://doi.acm.org/10.1145/125489.125473

[69] J. Voas, G. McGraw, Software Fault Injection: Inoculating Programs Against Errors, John Wiley \& Sons, 1997.

[70] R. Gopinath, C. Jensen, A. Groce, Mutations: How close are they to real faults?, in: 25th IEEE International Symposium on Software Reliability Engineering, ISSRE 2014, Naples, Italy, November 3-6, 2014, 2014, pp. 189-200. doi:10.1109/ISSRE. 2014.40.

URL https://doi .org/10.1109/ISSRE.2014.40

[71] R. Gopinath, C. Jensen, A. Groce, The theory of composite faults, in: 2017 IEEE International Conference on Software Testing, Verification and Validation (ICST), 2017, pp. 47-57. doi:10.1109/ICST.2017.12.

[72] W. B. Langdon, M. Harman, Y. Jia, Efficient multi-objective higher order mutation testing with genetic programming, Journal of Systems and Software 83 (12) (2010) 2416-2430. doi:10.1016/j.jss.2010.07.027. URL https://doi.org/10.1016/j.jss.2010.07.027

[73] R. Geist, A. J. Offutt, F. C. H. Jr., Estimation and enhancement of realtime software reliability through mutation analysis, IEEE Trans. Computers 41 (5) (1992) 550-558. doi:10.1109/12.142681.

URL https://doi.org/10.1109/12.142681

[74] I. Ahmed, C. Jensen, A. Groce, P. E. McKenney, Applying mutation analysis on kernel test suites: An experience report, in: 2017 IEEE In- 
ternational Conference on Software Testing, Verification and Validation Workshops (ICSTW), 2017, pp. 110-115. doi:10.1109/ICSTW.2017.26.

[75] A. J. Offutt, S. D. Lee, An empirical evaluation of weak mutation, IEEE Trans. Software Eng. 20 (5) (1994) 337-344. doi:10.1109/32.286422. URL http://dx.doi.org/10.1109/32.286422

[76] G. Kaminski, P. Ammann, J. Offutt, Improving logic-based testing, Journal of Systems and Software 86 (8) (2013) 2002-2012. doi:10.1016/j. jss.2012.08.024.

URL http://dx.doi.org/10.1016/j.jss.2012.08.024

[77] P. Anbalagan, T. Xie, Automated generation of pointcut mutants for testing pointcuts in aspectj programs, in: 19th International Symposium on Software Reliability Engineering (ISSRE 2008), 11-14 November 2008, Seattle/Redmond, WA, USA, 2008, pp. 239-248. doi:10.1109/ISSRE. 2008.58.

URL https://doi .org/10.1109/ISSRE. 2008.58

[78] A. Estero-Botaro, F. Palomo-Lozano, I. Medina-Bulo, Mutation operators for ws-bpel 2.0, in: 21th International Conference on Software \& Systems Engineering and their Applications, 2008.

[79] A. Estero-Botaro, F. Palomo-Lozano, I. Medina-Bulo, Quantitative evaluation of mutation operators for WS-BPEL compositions, in: Third International Conference on Software Testing, Verification and Validation, ICST 2010, Paris, France, April 7-9, 2010, Workshops Proceedings, 2010, pp. 142-150. doi:10.1109/ICSTW.2010.36

URL https://doi.org/10.1109/ICSTW.2010.36

[80] J. Boubeta-Puig, I. Medina-Bulo, A. García-Domínguez, Analogies and differences between mutation operators for WS-BPEL 2.0 and other languages, in: Fourth IEEE International Conference on Software Testing, Verification and Validation, ICST 2012, Berlin, Germany, 21-25 March, 
2011, Workshop Proceedings, 2011, pp. 398-407. doi:10.1109/ICSTW. 2011.52 .

URL http://dx.doi.org/10.1109/ICSTW.2011.52

[81] S. Mirshokraie, A. Mesbah, K. Pattabiraman, Guided mutation testing for javascript web applications, IEEE Trans. Software Eng. 41 (5) (2015) 429-444. doi:10.1109/TSE.2014.2371458

URL https://doi.org/10.1109/TSE.2014.2371458

[82] P. Delgado-Prez, S. Segura, I. Medina-Bulo, Assessment of c++ objectoriented mutation operators: A selective mutation approach, Software Testing, Verification and Reliability 27 (4-5) (2017) n/a-n/a. doi:10. 1002/stvr.1630.

URL http://dx.doi.org/10.1002/stvr.1630

[83] J. Hu, N. Li, J. Offutt, An analysis of OO mutation operators, in: Fourth IEEE International Conference on Software Testing, Verification and Validation, ICST 2012, Berlin, Germany, 21-25 March, 2011, Workshop Proceedings, 2011, pp. 334-341. doi:10.1109/ICSTW.2011.47.

URL http://dx.doi.org/10.1109/ICSTW.2011.47

[84] F. C. Ferrari, J. C. Maldonado, A. Rashid, Mutation testing for aspectoriented programs, in: First International Conference on Software Testing, Verification, and Validation, ICST 2008, Lillehammer, Norway, April 911, 2008, 2008, pp. 52-61. doi:10.1109/ICST.2008.37.

URL http://dx.doi .org/10.1109/ICST .2008.37

[85] L. Bottaci, Type sensitive application of mutation operators for dynamically typed programs, in: Third International Conference on Software Testing, Verification and Validation, ICST 2010, Paris, France, April 7-9, 2010, Workshops Proceedings, 2010, pp. 126-131. doi:10.1109/ICSTW. 2010.56 .

URL https://doi.org/10.1109/ICSTW.2010.56 
[86] M. Gligoric, S. Badame, R. Johnson, Smutant: a tool for type-sensitive mutation testing in a dynamic language, in: SIGSOFT/FSE'11 19th ACM SIGSOFT Symposium on the Foundations of Software Engineering (FSE-19) and ESEC'11: 13th European Software Engineering Conference (ESEC-13), Szeged, Hungary, September 5-9, 2011, 2011, pp. 424-427. doi:10.1145/2025113.2025181.

URL http://doi .acm.org/10.1145/2025113.2025181

[87] A. D. B. Alberto, A. Cavalcanti, M. Gaudel, A. Simão, Formal mutation testing for circus, Information \& Software Technology 81 (2017) 131-153. doi:10.1016/j.infsof.2016.04.003. URL https://doi.org/10.1016/j.infsof .2016.04.003

[88] U. Praphamontripong, J. Offutt, Applying mutation testing to web applications, in: Third International Conference on Software Testing, Verification and Validation, ICST 2010, Paris, France, April 7-9, 2010, Workshops Proceedings, 2010, pp. 132-141. doi:10.1109/ICSTW.2010.38.

URL http://dx.doi.org/10.1109/ICSTW .2010.38

[89] U. Praphamontripong, J. Offutt, L. Deng, J. Gu, An experimental evaluation of web mutation operators, in: Ninth IEEE International Conference on Software Testing, Verification and Validation Workshops, ICST Workshops 2016, Chicago, IL, USA, April 11-15, 2016, 2016, pp. 102-111. doi:10.1109/ICSTW.2016.17.

URL http://dx.doi.org/10.1109/ICSTW.2016.17

[90] U. Praphamontripong, J. Offutt, Finding redundancy in web mutation operators, in: 2017 IEEE International Conference on Software Testing, Verification and Validation Workshops (ICSTW), 2017, pp. 134-142. doi: 10.1109/ICSTW.2017.30.

[91] L. Deng, N. Mirzaei, P. Ammann, J. Offutt, Towards mutation analysis of android apps, in: Eighth IEEE International Conference on Software Testing, Verification and Validation, ICST 2015 Workshops, Graz, Austria, 
April 13-17, 2015, 2015, pp. 1-10. doi:10.1109/ICSTW.2015.7107450.

URL http://dx.doi.org/10.1109/ICSTW.2015.7107450

[92] L. Deng, J. Offutt, P. Ammann, N. Mirzaei, Mutation operators for testing android apps, Information \& Software Technology 81 (2017) 154-168. doi:10.1016/j.infsof.2016.04.012.

URL https://doi.org/10.1016/j.infsof .2016.04.012

[93] M. P. Usaola, G. Rojas, I. Rodrguez, S. Hernndez, An architecture for the development of mutation operators, in: 2017 IEEE International Conference on Software Testing, Verification and Validation Workshops (ICSTW), 2017, pp. 143-148. doi:10.1109/ICSTW.2017.31.

[94] M. Linares-Vásquez, G. Bavota, M. Tufano, K. Moran, M. Di Penta, C. Vendome, C. Bernal-Cárdenas, D. Poshyvanyk, Enabling mutation testing for android apps, in: Proceedings of the 2017 11th Joint Meeting on Foundations of Software Engineering, ESEC/FSE 2017, ACM, New York, NY, USA, 2017, pp. 233-244. doi:10.1145/3106237.3106275. URL http://doi.acm.org/10.1145/3106237.3106275

[95] R. A. P. Oliveira, E. Alégroth, Z. Gao, A. M. Memon, Definition and evaluation of mutation operators for gui-level mutation analysis, in: Eighth IEEE International Conference on Software Testing, Verification and Validation, ICST 2015 Workshops, Graz, Austria, April 13-17, 2015, 2015, pp. 1-10. doi:10.1109/ICSTW.2015.7107457

URL https://doi.org/10.1109/ICSTW.2015.7107457

[96] V. Lelli, A. Blouin, B. Baudry, Classifying and qualifying GUI defects, CoRR abs/1703.09567.

URL http://arxiv.org/abs/1703.09567

[97] R. Abraham, M. Erwig, Mutation operators for spreadsheets, IEEE Trans. Software Eng. 35 (1) (2009) 94-108. doi:10.1109/TSE.2008.73

URL https://doi .org/10.1109/TSE.2008.73 
[98] H. Dan, R. M. Hierons, Semantic mutation analysis of floating-point comparison, in: Fifth IEEE International Conference on Software Testing, Verification and Validation, ICST 2012, Montreal, QC, Canada, April 1721, 2012, 2012, pp. 290-299. doi:10.1109/ICST.2012.109.

URL http://dx.doi.org/10.1109/ICST.2012.109

[99] V. Jagannath, M. Gligoric, S. Lauterburg, D. Marinov, G. Agha, Mutation operators for actor systems, in: Third International Conference on Software Testing, Verification and Validation, ICST 2010, Paris, France, April 7-9, 2010, Workshops Proceedings, 2010, pp. 157-162. doi:10.1109/ICSTW.2010.6

URL http://dx.doi.org/10.1109/ICSTW.2010.6

[100] Y. Maezawa, K. Nishiura, H. Washizaki, S. Honiden, Validating ajax applications using a delay-based mutation technique, in: ACM/IEEE International Conference on Automated Software Engineering, ASE '14, Vasteras, Sweden - September 15 - 19, 2014, 2014, pp. 491-502. doi: $10.1145 / 2642937.2642996$

URL http://doi .acm.org/10.1145/2642937.2642996

[101] T. Xie, N. Tillmann, J. de Halleux, W. Schulte, Mutation analysis of parameterized unit tests, in: Second International Conference on Software Testing Verification and Validation, ICST 2009, Denver, Colorado, USA, April 1-4, 2009, Workshops Proceedings, 2009, pp. 177-181. doi:10. 1109/ICSTW. 2009.43

URL http://dx.doi.org/10.1109/ICSTW.2009.43

[102] D. B. Brown, M. Vaughn, B. Liblit, T. Reps, The care and feeding of wild-caught mutants, in: Proceedings of the 2017 11th Joint Meeting on Foundations of Software Engineering, ESEC/FSE 2017, ACM, New York, NY, USA, 2017, pp. 511-522. doi:10.1145/3106237.3106280.

URL http://doi .acm.org/10.1145/3106237.3106280

[103] J. Nanavati, F. Wu, M. Harman, Y. Jia, J. Krinke, Mutation testing 
of memory-related operators, in: Eighth IEEE International Conference on Software Testing, Verification and Validation, ICST 2015 Workshops, Graz, Austria, April 13-17, 2015, 2015, pp. 1-10. doi:10.1109/ICSTW. 2015.7107449 .

URL https://doi.org/10.1109/ICSTW.2015.7107449

[104] F. Wu, J. Nanavati, M. Harman, Y. Jia, J. Krinke, Memory mutation testing, Information \& Software Technology 81 (2017) 97-111. doi:10. 1016/j.infsof.2016.03.002. URL https://doi.org/10.1016/j.inf sof .2016.03.002

[105] B. J. Garvin, M. B. Cohen, Feature interaction faults revisited: An exploratory study, in: IEEE 22nd International Symposium on Software Reliability Engineering, ISSRE 2011, Hiroshima, Japan, November 29 December 2, 2011, 2011, pp. 90-99. doi:10.1109/ISSRE.2011.25

URL https://doi.org/10.1109/ISSRE.2011.25

[106] M. Al-Hajjaji, F. Benduhn, T. Thüm, T. Leich, G. Saake, Mutation operators for preprocessor-based variability, in: Proceedings of the Tenth International Workshop on Variability Modelling of Software-intensive Systems, Salvador, Brazil, January 27 - 29, 2016, 2016, pp. 81-88. doi:10.1145/2866614.2866626. URL http://doi . acm .org/10.1145/2866614.2866626

[107] M. E. Delamaro, J. Offutt, P. Ammann, Designing deletion mutation operators, in: Seventh IEEE International Conference on Software Testing, Verification and Validation, ICST 2014, March 31 2014-April 4, 2014, Cleveland, Ohio, USA, 2014, pp. 11-20. doi:10.1109/ICST.2014.12 URL http://dx.doi.org/10.1109/ICST .2014.12

[108] F. Belli, M. Beyazit, T. Takagi, Z. Furukawa, Mutation testing of "goback" functions based on pushdown automata, in: Fourth IEEE International Conference on Software Testing, Verification and Validation, ICST 2011, Berlin, Germany, March 21-25, 2011, 2011, pp. 249-258. 
doi:10.1109/ICST.2011.30,

URL https://doi .org/10.1109/ICST.2011.30

[109] R. Gopinath, E. Walkingshaw, How good are your types? using mutation analysis to evaluate the effectiveness of type annotations, in: 2017 IEEE International Conference on Software Testing, Verification and Validation Workshops (ICSTW), 2017, pp. 122-127. doi:10.1109/ICSTW.2017.28.

[110] R. Jabbarvand, S. Malek, mudroid: An energy-aware mutation testing framework for android, in: Proceedings of the 2017 11th Joint Meeting on Foundations of Software Engineering, ESEC/FSE 2017, ACM, New York, NY, USA, 2017, pp. 208-219. doi:10.1145/3106237.3106244.

URL http://doi .acm .org/10.1145/3106237.3106244

[111] P. Arcaini, A. Gargantini, E. Riccobene, Mutrex: A mutation-based generator of fault detecting strings for regular expressions, in: 2017 IEEE International Conference on Software Testing, Verification and Validation Workshops (ICSTW), 2017, pp. 87-96. doi:10.1109/ICSTW.2017.23.

[112] L. Zhang, S. Hou, J. Hu, T. Xie, H. Mei, Is operator-based mutant selection superior to random mutant selection?, in: Proceedings of the $32 \mathrm{nd}$ ACM/IEEE International Conference on Software Engineering - Volume 1, ICSE 2010, Cape Town, South Africa, 1-8 May 2010, 2010, pp. 435-444. doi:10.1145/1806799.1806863.

URL http://doi .acm.org/10.1145/1806799.1806863

[113] R. Gopinath, A. Alipour, I. Ahmed, C. Jensen, A. Groce, How hard does mutation analysis have to be, anyway?, in: 26th IEEE International Symposium on Software Reliability Engineering, ISSRE 2015, Gaithersbury, MD, USA, November 2-5, 2015, 2015, pp. 216-227. doi: 10.1109/ISSRE. 2015.7381815.

URL https: //doi .org/10.1109/ISSRE.2015.7381815

[114] A. S. Namin, J. H. Andrews, D. J. Murdoch, Sufficient mutation operators for measuring test effectiveness, in: 30th International Conference on 
Software Engineering (ICSE 2008), Leipzig, Germany, May 10-18, 2008, 2008, pp. 351-360. doi:10.1145/1368088.1368136.

URL http://doi.acm.org/10.1145/1368088.1368136

[115] M. E. Delamaro, L. Deng, V. H. S. Durelli, N. Li, J. Offutt, Experimental evaluation of SDL and one-op mutation for C, in: Seventh IEEE International Conference on Software Testing, Verification and Validation, ICST 2014, March 31 2014-April 4, 2014, Cleveland, Ohio, USA, 2014, pp. 203212. doi:10.1109/ICST.2014.33. URL http://dx.doi.org/10.1109/ICST.2014.33

[116] V. H. S. Durelli, N. M. D. Souza, M. E. Delamaro, Are deletion mutants easier to identify manually?, in: 2017 IEEE International Conference on Software Testing, Verification and Validation Workshops (ICSTW), 2017, pp. 149-158. doi:10.1109/ICSTW.2017.32.

[117] X. Yao, M. Harman, Y. Jia, A study of equivalent and stubborn mutation operators using human analysis of equivalence, in: 36th International Conference on Software Engineering, ICSE '14, Hyderabad, India - May 31 - June 07, 2014, 2014, pp. 919-930. doi:10.1145/2568225.2568265 URL http://doi.acm.org/10.1145/2568225.2568265

[118] J. Zhang, M. Zhu, D. Hao, L. Zhang, An empirical study on the scalability of selective mutation testing, in: 25th IEEE International Symposium on Software Reliability Engineering, ISSRE 2014, Naples, Italy, November 3-6, 2014, 2014, pp. 277-287. doi:10.1109/ISSRE.2014.27. URL https://doi.org/10.1109/ISSRE.2014.27

[119] J. Zhang, Scalability studies on selective mutation testing, in: 37th IEEE/ACM International Conference on Software Engineering, ICSE 2015, Florence, Italy, May 16-24, 2015, Volume 2, 2015, pp. 851-854. doi:10.1109/ICSE.2015.276. URL https://doi.org/10.1109/ICSE.2015.276 
[120] L. Zhang, M. Gligoric, D. Marinov, S. Khurshid, Operator-based and random mutant selection: Better together, in: 2013 28th IEEE/ACM International Conference on Automated Software Engineering, ASE 2013, Silicon Valley, CA, USA, November 11-15, 2013, 2013, pp. 92-102. doi: 10.1109/ASE. 2013.6693070

URL http://dx . doi .org/10.1109/ASE . 2013.6693070

[121] R. Gopinath, M. A. Alipour, I. Ahmed, C. Jensen, A. Groce, On the limits of mutation reduction strategies, in: Proceedings of the 38th International Conference on Software Engineering, ICSE 2016, Austin, TX, USA, May 14-22, 2016, 2016, pp. 511-522. doi:10.1145/2884781.2884787. URL http://doi .acm.org/10.1145/2884781.2884787

[122] B. Kurtz, P. Ammann, J. Offutt, M. E. Delamaro, M. Kurtz, N. Gökçe, Analyzing the validity of selective mutation with dominator mutants, in: Proceedings of the 24th ACM SIGSOFT International Symposium on Foundations of Software Engineering, FSE 2016, Seattle, WA, USA, November 13-18, 2016, 2016, pp. 571-582. doi:10.1145/2950290. 2950322 .

URL http://doi .acm . org/10.1145/2950290.2950322

[123] M. Gligoric, L. Zhang, C. Pereira, G. Pokam, Selective mutation testing for concurrent code, in: International Symposium on Software Testing and Analysis, ISSTA '13, Lugano, Switzerland, July 15-20, 2013, 2013, pp. 224-234. doi:10.1145/2483760.2483773. URL http://doi .acm .org/10.1145/2483760.2483773

[124] G. K. Kaminski, P. Ammann, Using a fault hierarchy to improve the efficiency of DNF logic mutation testing, in: Second International Conference on Software Testing Verification and Validation, ICST 2009, Denver, Colorado, USA, April 1-4, 2009, 2009, pp. 386-395. doi:10.1109/ICST. 2009.13 .

URL http://dx.doi .org/10.1109/ICST.2009.13 
[125] M. Papadakis, Y. L. Traon, Effective fault localization via mutation analysis: a selective mutation approach, in: Symposium on Applied Computing, SAC 2014, Gyeongju, Republic of Korea - March 24 - 28, 2014, 2014, pp. 1293-1300. doi:10.1145/2554850.2554978.

URL http://doi .acm.org/10.1145/2554850.2554978

[126] M. Polo, M. Piattini, I. G. R. de Guzmán, Decreasing the cost of mutation testing with second-order mutants, Softw. Test., Verif. Reliab. 19 (2) (2009) 111-131. doi:10.1002/stvr.392

URL http://dx.doi.org/10.1002/stvr.392

[127] M. Papadakis, N. Malevris, M. Kintis, Mutation testing strategies - A collateral approach, in: ICSOFT 2010 - Proceedings of the Fifth International Conference on Software and Data Technologies, Volume 2, Athens, Greece, July 22-24, 2010, 2010, pp. 325-328.

[128] P. R. Mateo, M. P. Usaola, J. L. F. Alemán, Validating second-order mutation at system level, IEEE Trans. Software Eng. 39 (4) (2013) 570587. doi:10.1109/TSE.2012.39.

URL https://doi.org/10.1109/TSE.2012.39

[129] A. Parsai, A. Murgia, S. Demeyer, A model to estimate first-order mutation coverage from higher-order mutation coverage, in: 2016 IEEE International Conference on Software Quality, Reliability and Security, QRS 2016, Vienna, Austria, August 1-3, 2016, 2016, pp. 365-373. doi: 10.1109/QRS.2016.48.

URL https://doi.org/10.1109/QRS.2016.48

[130] R. Just, B. Kurtz, P. Ammann, Inferring mutant utility from program context, in: Proceedings of the 26th ACM SIGSOFT International Symposium on Software Testing and Analysis, Santa Barbara, CA, USA, July 10 - 14, 2017, 2017, pp. 284-294. doi:10.1145/3092703.3092732

URL http://doi . acm .org/10.1145/3092703.3092732 
[131] C. Sun, F. Xue, H. Liu, X. Zhang, A path-aware approach to mutant reduction in mutation testing, Information \& Software Technology 81 (2017)

65-81. doi:10.1016/j.infsof.2016.02.006.

URL https://doi.org/10.1016/j.infsof .2016.02.006

[132] D. Gong, G. Zhang, X. Yao, F. Meng, Mutant reduction based on dominance relation for weak mutation testing, Information \& Software Technology 81 (2017) 82-96. doi:10.1016/j.infsof.2016.05.001.

URL https://doi .org/10.1016/j . inf sof .2016.05.001

[133] C. Iida, S. Takada, Reducing mutants with mutant killable precondition, in: 2017 IEEE International Conference on Software Testing, Verification and Validation Workshops (ICSTW), 2017, pp. 128-133. doi:10.1109/ ICSTW.2017.29.

[134] M. Patrick, M. Oriol, J. A. Clark, MESSI: mutant evaluation by static semantic interpretation, in: Fifth IEEE International Conference on Software Testing, Verification and Validation, ICST 2012, Montreal, QC, Canada, April 17-21, 2012, 2012, pp. 711-719. doi:10.1109/ICST.2012. 161 .

URL https://doi.org/10.1109/ICST.2012.161

[135] M. Patrick, R. Alexander, M. Oriol, J. A. Clark, Probability-based semantic interpretation of mutants, in: Seventh IEEE International Conference on Software Testing, Verification and Validation, ICST 2014 Workshops Proceedings, March 31 - April 4, 2014, Cleveland, Ohio, USA, 2014, pp. 186-195. doi:10.1109/ICSTW.2014.18.

URL https://doi.org/10.1109/ICSTW.2014.18

[136] M. Sridharan, A. S. Namin, Prioritizing mutation operators based on importance sampling, in: IEEE 21st International Symposium on Software Reliability Engineering, ISSRE 2010, San Jose, CA, USA, 1-4 November 2010, 2010, pp. 378-387. doi:10.1109/ISSRE.2010.16.

URL http://dx.doi .org/10.1109/ISSRE.2010.16 
[137] A. S. Namin, X. Xue, O. Rosas, P. Sharma, Muranker: a mutant ranking tool, Softw. Test., Verif. Reliab. 25 (5-7) (2015) 572-604. doi:10.1002/ stvr.1542.

URL http://dx.doi.org/10.1002/stvr.1542

[138] J. Nam, D. Schuler, A. Zeller, Calibrated mutation testing, in: Fourth IEEE International Conference on Software Testing, Verification and Validation, ICST 2012, Berlin, Germany, 21-25 March, 2011, Workshop Proceedings, 2011, pp. 376-381. doi:10.1109/ICSTW.2011.57.

URL https://doi .org/10.1109/ICSTW.2011.57

[139] L. Inozemtseva, H. Hemmati, R. Holmes, Using fault history to improve mutation reduction, in: Joint Meeting of the European Software Engineering Conference and the ACM SIGSOFT Symposium on the Foundations of Software Engineering, ESEC/FSE'13, Saint Petersburg, Russian Federation, August 18-26, 2013, 2013, pp. 639-642. doi:10.1145/2491411. 2494586.

URL http://doi.acm.org/10.1145/2491411.2494586

[140] R. H. Untch, A. J. Offutt, M. J. Harrold, Mutation analysis using mutant schemata, in: Proceedings of the 1993 International Symposium on Software Testing and Analysis, ISSTA 1993, Cambridge, MA, USA, June 28-30, 1993, 1993, pp. 139-148. doi:10.1145/154183.154265.

URL http://doi .acm.org/10.1145/154183.154265

[141] M. Papadakis, N. Malevris, Automatic mutation test case generation via dynamic symbolic execution, in: IEEE 21st International Symposium on Software Reliability Engineering, ISSRE 2010, San Jose, CA, USA, 1-4 November 2010, 2010, pp. 121-130. doi:10.1109/ISSRE.2010.38

URL http://dx.doi.org/10.1109/ISSRE.2010.38

[142] B. Wang, Y. Xiong, Y. Shi, L. Zhang, D. Hao, Faster mutation analysis via equivalence modulo states (2017) 295-306 doi:10.1145/3092703. 
3092714 .

URL http://doi .acm.org/10.1145/3092703.3092714

[143] S. Tokumoto, H. Yoshida, K. Sakamoto, S. Honiden, Muvm: Higher order mutation analysis virtual machine for C in: 2016 IEEE International Conference on Software Testing, Verification and Validation, ICST 2016, Chicago, IL, USA, April 11-15, 2016, 2016, pp. 320-329. doi:10.1109/ ICST.2016.18,

URL http://dx.doi.org/10.1109/ICST .2016.18

[144] S. Bardin, M. Delahaye, R. David, N. Kosmatov, M. Papadakis, Y. L. Traon, J. Marion, Sound and quasi-complete detection of infeasible test requirements, in: 8th IEEE International Conference on Software Testing, Verification and Validation, ICST 2015, Graz, Austria, April 13-17, 2015, 2015, pp. 1-10. doi:10.1109/ICST.2015.7102607. URL http://dx.doi .org/10.1109/ICST . 2015.7102607

[145] M. Marcozzi, S. Bardin, N. Kosmatov, M. Papadakis, V. Prevosto, L. Correnson, Time to clean your test objectives, in: Proceedings of the 40th International Conference on Software Engineering, ICSE 2018, Gothenburg, Sweden, May 27 - 3 June, 2018, 2018.

[146] M. Marcozzi, M. Delahaye, S. Bardin, N. Kosmatov, V. Prevosto, Generic and effective specification of structural test objectives, in: 2017 IEEE International Conference on Software Testing, Verification and Validation (ICST), 2017, pp. 436-441. doi:10.1109/ICST.2017.48.

[147] H. Coles, T. Laurent, C. Henard, M. Papadakis, A. Ventresque, PIT: a practical mutation testing tool for java (demo), in: Proceedings of the 25th International Symposium on Software Testing and Analysis, ISSTA 2016, Saarbrücken, Germany, July 18-20, 2016, 2016, pp. 449-452. doi: 10.1145/2931037.2948707. URL http://doi . acm.org/10.1145/2931037.2948707 
[148] F. Hariri, A. Shi, H. Converse, S. Khurshid, D. Marinov, Evaluating the effects of compiler optimizations on mutation testing at the compiler IR level, in: 27th IEEE International Symposium on Software Reliability Engineering, ISSRE 2016, Ottawa, ON, Canada, October 23-27, 2016, 2016, pp. 105-115. doi:10.1109/ISSRE.2016.51.

URL http://dx.doi.org/10.1109/ISSRE.2016.51

[149] M. Papadakis, N. Malevris, Mutation based test case generation via a path selection strategy, Information \& Software Technology 54 (9) (2012) 915-932. doi:10.1016/j.infsof .2012.02.004. URL http://dx.doi.org/10.1016/j.infsof .2012.02.004

[150] V. H. S. Durelli, J. Offutt, M. E. Delamaro, Toward harnessing high-level language virtual machines for further speeding up weak mutation testing, in: Fifth IEEE International Conference on Software Testing, Verification and Validation, ICST 2012, Montreal, QC, Canada, April 17-21, 2012, 2012, pp. 681-690. doi:10.1109/ICST.2012.158

URL http://dx.doi.org/10.1109/ICST.2012.158

[151] X. Devroey, G. Perrouin, M. Cordy, M. Papadakis, A. Legay, P. Schobbens, A variability perspective of mutation analysis, in: Proceedings of the 22nd ACM SIGSOFT International Symposium on Foundations of Software Engineering, (FSE-22), Hong Kong, China, November 16 - 22, 2014, 2014, pp. 841-844. doi:10.1145/2635868.2666610. URL http://doi .acm.org/10.1145/2635868.2666610

[152] M. Kintis, M. Papadakis, Y. Jia, N. Malevris, Y. L. Traon, M. Harman, Detecting trivial mutant equivalences via compiler optimisations, IEEE Transactions on Software Engineering PP (99) (2017) 1-1. doi:10.1109/ TSE.2017.2684805

[153] M. Kintis, Effective methods to tackle the equivalent mutant problem when testing software with mutation, Ph.D. thesis, Department of Informatics, Athens University of Economics and Business (2016). 
[154] M. Kintis, N. Malevris, Using data flow patterns for equivalent mutant detection, in: Seventh IEEE International Conference on Software Testing, Verification and Validation, ICST 2014 Workshops Proceedings, March 31 - April 4, 2014, Cleveland, Ohio, USA, 2014, pp. 196-205. doi:10.1109/ ICSTW.2014.21.

URL http://dx.doi.org/10.1109/ICSTW.2014.21

[155] M. Kintis, N. Malevris, MEDIC: A static analysis framework for equivalent mutant identification, Information \& Software Technology 68 (2015) 1-17. doi:10.1016/j.infsof.2015.07.009.

URL https://doi.org/10.1016/j .inf sof .2015.07.009

[156] D. Holling, S. Banescu, M. Probst, A. Petrovska, A. Pretschner, Nequivack: Assessing mutation score confidence, in: Ninth IEEE International Conference on Software Testing, Verification and Validation Workshops, ICST Workshops 2016, Chicago, IL, USA, April 11-15, 2016, 2016, pp. 152-161. doi:10.1109/ICSTW.2016.29.

URL https://doi .org/10.1109/ICSTW.2016.29

[157] M. Kintis, N. Malevris, Identifying more equivalent mutants via code similarity, in: 20th Asia-Pacific Software Engineering Conference, APSEC 2013, Ratchathewi, Bangkok, Thailand, December 2-5, 2013 - Volume 1, 2013, pp. 180-188. doi:10.1109/APSEC.2013.34.

URL https://doi .org/10.1109/APSEC.2013.34

[158] K. A. Foster, Error sensitive test cases analysis (ESTCA), IEEE Trans. Software Eng. 6 (3) (1980) 258-264. doi:10.1109/TSE.1980.234487. URL https://doi.org/10.1109/TSE.1980.234487

[159] W. E. Howden, Weak mutation testing and completeness of test sets, IEEE Trans. Software Eng. 8 (4) (1982) 371-379. doi:10.1109/TSE. 1982.235571

URL https://doi.org/10.1109/TSE.1982.235571 
[160] K. Tai, Predicate-based test generation for computer programs, in: Proceedings of the 15th International Conference on Software Engineering, Baltimore, Maryland, USA, May 17-21, 1993., 1993, pp. 267-276.

URL http://portal . acm.org/citation. cfm?id=257572.257631

[161] K. Tai, Theory of fault-based predicate testing for computer programs, IEEE Trans. Software Eng. 22 (8) (1996) 552-562. doi:10.1109/32. 536956 .

URL https://doi.org/10.1109/32.536956

[162] M. Papadakis, Error detection methods in java programs using the mutation method, Masters thesis, Athens University of Economics and Business (June 2005).

[163] G. Kaminski, P. Ammann, J. Offutt, Better predicate testing, in: Proceedings of the 6th International Workshop on Automation of Software Test, AST 2011, Waikiki, Honolulu, HI, USA, May 23-24, 2011, 2011, pp. 57-63. doi:10.1145/1982595.1982608. URL http://doi .acm.org/10.1145/1982595.1982608

[164] R. Just, G. M. Kapfhammer, F. Schweiggert, Do redundant mutants affect the effectiveness and efficiency of mutation analysis?, in: Fifth IEEE International Conference on Software Testing, Verification and Validation, ICST 2012, Montreal, QC, Canada, April 17-21, 2012, 2012, pp. 720-725. doi:10.1109/ICST.2012.162.

URL http://dx.doi.org/10.1109/ICST.2012.162

[165] R. Just, F. Schweiggert, Higher accuracy and lower run time: efficient mutation analysis using non-redundant mutation operators, Softw. Test., Verif. Reliab. 25 (5-7) (2015) 490-507. doi:10.1002/stvr.1561.

URL http://dx.doi.org/10.1002/stvr.1561

[166] L. Fernandes, M. Ribeiro, L. Carvalho, R. Gheyi, M. Mongiovi, A. Santos, A. Cavalcanti, F. Ferrari, J. C. Maldonado, Avoiding useless mutants, 
in: Proceedings of the 16th ACM SIGPLAN International Conference on Generative Programming: Concepts and Experiences, GPCE 2017, ACM, New York, NY, USA, 2017, pp. 187-198. doi:10.1145/3136040.3136053

[167] T. Laurent, A. Ventresque, M. Papadakis, C. Henard, Y. L. Traon, Assessing and improving the mutation testing practice of PIT, CoRR abs/1601.02351.

URL http://arxiv .org/abs/1601.02351

[168] B. Lindström, A. Marki, On strong mutation and subsuming mutants, in: Ninth IEEE International Conference on Software Testing, Verification and Validation Workshops, ICST Workshops 2016, Chicago, IL, USA, April 11-15, 2016, 2016, pp. 112-121. doi:10.1109/ICSTW.2016.28

URL http://dx.doi.org/10.1109/ICSTW.2016.28

[169] B. Kurtz, P. Ammann, J. Offutt, Static analysis of mutant subsumption, in: Eighth IEEE International Conference on Software Testing, Verification and Validation, ICST 2015 Workshops, Graz, Austria, April 13-17, 2015, 2015, pp. 1-10. doi:10.1109/ICSTW.2015.7107454.

URL https://doi.org/10.1109/ICSTW.2015.7107454

[170] R. A. DeMillo, A. J. Offutt, Constraint-based automatic test data generation, IEEE Trans. Software Eng. 17 (9) (1991) 900-910. doi:10.1109/ 32.92910 .

URL https ://doi.org/10.1109/32.92910

[171] G. Fraser, A. Zeller, Mutation-driven generation of unit tests and oracles IEEE Trans. Software Eng. 38 (2) (2012) 278-292. doi:10.1109/TSE. 2011.93.

URL https://doi.org/10.1109/TSE.2011.93

[172] M. Harman, Y. Jia, W. B. Langdon, Strong higher order mutationbased test data generation, in: SIGSOFT/FSE'11 19th ACM SIGSOFT Symposium on the Foundations of Software Engineering (FSE-19) and 
ESEC'11: 13th European Software Engineering Conference (ESEC-13), Szeged, Hungary, September 5-9, 2011, 2011, pp. 212-222. doi:10.1145/ 2025113.2025144 .

URL http://doi.acm.org/10.1145/2025113.2025144

[173] S. Anand, E. K. Burke, T. Y. Chen, J. A. Clark, M. B. Cohen, W. Grieskamp, M. Harman, M. J. Harrold, P. McMinn, An orchestrated survey of methodologies for automated software test case generation, Journal of Systems and Software 86 (8) (2013) 1978-2001. doi: $10.1016 / \mathrm{j} \cdot \mathrm{jss} .2013 .02 .061$. URL https://doi.org/10.1016/j.jss.2013.02.061

[174] F. Wotawa, M. Nica, B. K. Aichernig, Generating distinguishing tests using the minion constraint solver, in: Third International Conference on Software Testing, Verification and Validation, ICST 2010, Paris, France, April 7-9, 2010, Workshops Proceedings, 2010, pp. 325-330. doi:10. 1109/ICSTW.2010.11.

URL http://dx.doi.org/10.1109/ICSTW .2010.11

[175] S. Nica, On the improvement of the mutation score using distinguishing test cases, in: Fourth IEEE International Conference on Software Testing, Verification and Validation, ICST 2011, Berlin, Germany, March 21-25, 2011, 2011, pp. 423-426. doi:10.1109/ICST.2011.40.

URL https://doi.org/10.1109/ICST.2011.40

[176] M. Papadakis, N. Malevris, An effective path selection strategy for mutation testing, in: 16th Asia-Pacific Software Engineering Conference, APSEC 2009, 1-3 December 2009, Batu Ferringhi, Penang, Malaysia, 2009, pp. 422-429. doi:10.1109/APSEC.2009.68

URL https://doi.org/10.1109/APSEC.2009.68

[177] M. Papadakis, N. Malevris, Automatically performing weak mutation with the aid of symbolic execution, concolic testing and search-based testing, Software Quality Journal 19 (4) (2011) 691-723. doi:10.1007/ 
s11219-011-9142-y

URL http://dx.doi .org/10.1007/s11219-011-9142-y

[178] S. Anand, C. S. Pasareanu, W. Visser, JPF-SE: A symbolic execution extension to java pathfinder, in: Tools and Algorithms for the Construction and Analysis of Systems, 13th International Conference, TACAS 2007, Held as Part of the Joint European Conferences on Theory and Practice of Software, ETAPS 2007 Braga, Portugal, March 24 - April 1, 2007, Proceedings, 2007, pp. 134-138. doi:10.1007/978-3-540-71209-1_12.

URL https://doi .org/10.1007/978-3-540-71209-1_12

[179] C. Cadar, D. Dunbar, D. R. Engler, KLEE: unassisted and automatic generation of high-coverage tests for complex systems programs, in: 8th USENIX Symposium on Operating Systems Design and Implementation, OSDI 2008, December 8-10, 2008, San Diego, California, USA, Proceedings, 2008, pp. 209-224.

URL http://wWw.usenix.org/events/osdi08/tech/full_papers/ cadar/cadar.pdf

[180] H. Riener, R. Bloem, G. Fey, Test case generation from mutants using model checking techniques, in: Fourth IEEE International Conference on Software Testing, Verification and Validation, ICST 2012, Berlin, Germany, 21-25 March, 2011, Workshop Proceedings, 2011, pp. 388-397. doi:10.1109/ICSTW.2011.55.

URL http://dx.doi.org/10.1109/ICSTW.2011.55

[181] M. Papadakis, N. Malevris, M. Kallia, Towards automating the generation of mutation tests, in: The 5th Workshop on Automation of Software Test, AST 2010, May 3-4, 2010, Cape Town, South Africa, 2010, pp. 111-118. doi:10.1145/1808266.1808283.

URL http://doi .acm .org/10.1145/1808266.1808283

[182] L. Zhang, T. Xie, L. Zhang, N. Tillmann, J. de Halleux, H. Mei, Test generation via dynamic symbolic execution for mutation testing, in: 26th 
IEEE International Conference on Software Maintenance (ICSM 2010), September 12-18, 2010, Timisoara, Romania, 2010, pp. 1-10. doi:10. 1109/ICSM.2010.5609672

URL https://doi.org/10.1109/ICSM.2010.5609672

[183] S. Bardin, N. Kosmatov, F. Cheynier, Efficient leveraging of symbolic execution to advanced coverage criteria, in: Seventh IEEE International Conference on Software Testing, Verification and Validation, ICST 2014, March 31 2014-April 4, 2014, Cleveland, Ohio, USA, 2014, pp. 173-182. doi:10.1109/ICST.2014.30. URL http://dx.doi.org/10.1109/ICST.2014.30

[184] K. Jamrozik, G. Fraser, N. Tillmann, J. de Halleux, Augmented dynamic symbolic execution, in: IEEE/ACM International Conference on Automated Software Engineering, ASE'12, Essen, Germany, September 3-7, 2012, 2012, pp. 254-257. doi:10.1145/2351676.2351716.

URL http://doi .acm.org/10.1145/2351676.2351716

[185] F. C. M. Souza, M. Papadakis, Y. L. Traon, M. E. Delamaro, Strong mutation-based test data generation using hill climbing, in: Proceedings of the 9th International Workshop on Search-Based Software Testing, SBST@ICSE 2016, Austin, Texas, USA, May 14-22, 2016, 2016, pp. 4554. doi:10.1145/2897010.2897012. URL http://doi.acm.org/10.1145/2897010.2897012

[186] K. Ayari, S. Bouktif, G. Antoniol, Automatic mutation test input data generation via ant colony, in: Genetic and Evolutionary Computation Conference, GECCO 2007, Proceedings, London, England, UK, July 711, 2007, 2007, pp. 1074-1081. doi:10.1145/1276958.1277172. URL http://doi .acm.org/10.1145/1276958.1277172

[187] G. Fraser, A. Zeller, Mutation-driven generation of unit tests and oracles, in: Proceedings of the Nineteenth International Symposium on Software Testing and Analysis, ISSTA 2010, Trento, Italy, July 12-16, 2010, 2010, 
pp. 147-158. doi:10.1145/1831708.1831728.

URL http://doi .acm.org/10.1145/1831708.1831728

[188] M. Papadakis, N. Malevris, Automatic mutation based test data generation, in: 13th Annual Genetic and Evolutionary Computation Conference, GECCO 2011, Companion Material Proceedings, Dublin, Ireland, July 1216, 2011, 2011, pp. 247-248. doi:10.1145/2001858.2001997. URL http://doi .acm.org/10.1145/2001858.2001997

[189] M. Papadakis, N. Malevris, Killing mutants effectively a search based approach in: Knowledge-Based Software Engineering - Proceedings of the Tenth Conference on Knowledge-Based Software Engineering, JCKBSE 2012, Rodos, Greece, August 23-26, 2012, 2012, pp. 217-226. doi:10. 3233/978-1-61499-094-9-217 URL http://dx . doi .org/10.3233/978-1-61499-094-9-217

[190] M. Papadakis, N. Malevris, Searching and generating test inputs for mutation testing, SpringerPlus 2 (1) (2013) 121. doi:10.1186/ 2193-1801-2-121.

URL http://dx.doi.org/10.1186/2193-1801-2-121

[191] M. Patrick, R. Alexander, M. Oriol, J. A. Clark, Using mutation analysis to evolve subdomains for random testing, in: Sixth IEEE International Conference on Software Testing, Verification and Validation, ICST 2013 Workshops Proceedings, Luxembourg, Luxembourg, March 18-22, 2013, 2013, pp. 53-62. doi:10.1109/ICSTW.2013.14. URL http://dx.doi.org/10.1109/ICSTW .2013.14

[192] G. Fraser, A. Arcuri, Achieving scalable mutation-based generation of whole test suites, Empirical Software Engineering 20 (3) (2015) 783-812. doi:10.1007/s10664-013-9299-z URL http://dx . doi .org/10.1007/s10664-013-9299-z

[193] A. Shi, A. Gyori, M. Gligoric, A. Zaytsev, D. Marinov, Balancing tradeoffs in test-suite reduction, in: Proceedings of the 22nd ACM SIGSOFT 
International Symposium on Foundations of Software Engineering, (FSE22), Hong Kong, China, November 16 - 22, 2014, 2014, pp. 246-256. doi:10.1145/2635868.2635921.

URL http://doi.acm.org/10.1145/2635868.2635921

[194] Y. Lou, D. Hao, L. Zhang, Mutation-based test-case prioritization in software evolution, in: 26th IEEE International Symposium on Software Reliability Engineering, ISSRE 2015, Gaithersbury, MD, USA, November 2-5, 2015, 2015, pp. 46-57. doi:10.1109/ISSRE.2015.7381798

URL http://dx.doi.org/10.1109/ISSRE. 2015.7381798

[195] L. Zhang, D. Marinov, S. Khurshid, Faster mutation testing inspired by test prioritization and reduction, in: International Symposium on Software Testing and Analysis, ISSTA '13, Lugano, Switzerland, July 15-20, 2013, 2013, pp. 235-245. doi:10.1145/2483760.2483782

URL http://doi.acm.org/10.1145/2483760.2483782

[196] R. Just, G. M. Kapfhammer, F. Schweiggert, Using non-redundant mutation operators and test suite prioritization to achieve efficient and scalable mutation analysis, in: 23rd IEEE International Symposium on Software Reliability Engineering, ISSRE 2012, Dallas, TX, USA, November 27-30, 2012, 2012, pp. 11-20. doi:10.1109/ISSRE.2012.31

URL http://dx.doi.org/10.1109/ISSRE.2012.31

[197] Q. Zhu, A. Panichella, A. Zaidman, Speeding-up mutation testing via data compression and state infection, in: 2017 IEEE International Conference on Software Testing, Verification and Validation Workshops (ICSTW), 2017, pp. 103-109. doi:10.1109/ICSTW.2017.25.

[198] M. E. Delamaro, Proteum - A Mutation Analysis Based Testing Environmen, Masters thesis, University of São Paulo, Sao Paulo, Brazil (1993).

[199] S. Kim, Y. Ma, Y. R. Kwon, Combining weak and strong mutation for a noninterpretive java mutation system, Softw. Test., Verif. Reliab. 23 (8) 
(2013) 647-668. doi:10.1002/stvr.1480.

URL http://dx.doi.org/10.1002/stvr.1480

[200] R. Just, M. D. Ernst, G. Fraser, Efficient mutation analysis by propagating and partitioning infected execution states, in: International Symposium on Software Testing and Analysis, ISSTA '14, San Jose, CA, USA - July 21 - 26, 2014, 2014, pp. 315-326. doi:10.1145/2610384.2610388. URL http://doi .acm .org/10.1145/2610384.2610388

[201] P. R. Mateo, M. P. Usaola, Mutant execution cost reduction: Through MUSIC (mutant schema improved with extra code), in: Fifth IEEE International Conference on Software Testing, Verification and Validation, ICST 2012, Montreal, QC, Canada, April 17-21, 2012, 2012, pp. 664-672. doi:10.1109/ICST.2012.156.

URL http: //dx.doi.org/10.1109/ICST .2012.156

[202] P. R. Mateo, M. P. Usaola, Reducing mutation costs through uncovered mutants, Softw. Test., Verif. Reliab. 25 (5-7) (2015) 464-489. doi:10. $1002 /$ stvr. 1534 .

URL http://dx.doi .org/10.1002/stvr.1534

[203] K. N. King, A. J. Offutt, A fortran language system for mutation-based software testing, Softw., Pract. Exper. 21 (7) (1991) 685-718. doi:10. 1002/spe.4380210704.

URL https://doi.org/10.1002/spe.4380210704

[204] M. Papadakis, M. E. Delamaro, Y. L. Traon, Proteum/fl: A tool for localizing faults using mutation analysis, in: 13th IEEE International Working Conference on Source Code Analysis and Manipulation, SCAM 2013, Eindhoven, Netherlands, September 22-23, 2013, 2013, pp. 94-99. doi:10.1109/SCAM.2013.6648189.

URL http://dx .doi .org/10.1109/SCAM . 2013.6648189

[205] D. Schuler, A. Zeller, Javalanche: efficient mutation testing for java, in: Proceedings of the 7th joint meeting of the European Software Engineering 
Conference and the ACM SIGSOFT International Symposium on Foundations of Software Engineering, 2009, Amsterdam, The Netherlands, August 24-28, 2009, 2009, pp. 297-298. doi:10.1145/1595696.1595750.

URL http://doi.acm.org/10.1145/1595696.1595750

[206] P. R. Mateo, M. P. Usaola, Parallel mutation testing, Softw. Test., Verif. Reliab. 23 (4) (2013) 315-350. doi:10.1002/stvr.1471. URL http://dx.doi.org/10.1002/stvr.1471

[207] M. Gligoric, V. Jagannath, Q. Luo, D. Marinov, Efficient mutation testing of multithreaded code, Softw. Test., Verif. Reliab. 23 (5) (2013) 375-403. doi:10.1002/stvr.1469.

URL http://dx.doi.org/10.1002/stvr.1469

[208] M. Gligoric, V. Jagannath, D. Marinov, Mutmut: Efficient exploration for mutation testing of multithreaded code, in: Third International Conference on Software Testing, Verification and Validation, ICST 2010, Paris, France, April 7-9, 2010, 2010, pp. 55-64. doi:10.1109/ICST.2010.33. URL http://dx.doi .org/10.1109/ICST .2010.33

[209] P. Gong, R. Zhao, Z. Li, Faster mutation-based fault localization with a novel mutation execution strategy, in: Eighth IEEE International Conference on Software Testing, Verification and Validation, ICST 2015 Workshops, Graz, Austria, April 13-17, 2015, 2015, pp. 1-10. doi: 10.1109/ICSTW. 2015.7107448,

URL http://dx.doi.org/10.1109/ICSTW.2015.7107448

[210] L. Zhang, D. Marinov, L. Zhang, S. Khurshid, Regression mutation testing, in: International Symposium on Software Testing and Analysis, ISSTA 2012, Minneapolis, MN, USA, July 15-20, 2012, 2012, pp. 331-341. doi:10.1145/2338965.2336793.

URL http://doi . acm.org/10.1145/2338965.2336793

[211] C. J. Wright, G. M. Kapfhammer, P. McMinn, Efficient mutation analysis of relational database structure using mutant schemata and paral- 
lelisation, in: Sixth IEEE International Conference on Software Testing, Verification and Validation, ICST 2013 Workshops Proceedings, Luxembourg, Luxembourg, March 18-22, 2013, 2013, pp. 63-72. doi: 10.1109/ICSTW.2013.15.

URL http://dx.doi.org/10.1109/ICSTW.2013.15

[212] C. Zhou, P. G. Frankl, Inferential checking for mutants modifying database states, in: Fourth IEEE International Conference on Software Testing, Verification and Validation, ICST 2011, Berlin, Germany, March 21-25, 2011, 2011, pp. 259-268. doi:10.1109/ICST.2011.63.

URL http://dx.doi .org/10.1109/ICST .2011.63

[213] M. Patrick, A. P. Craig, N. J. Cunniffe, M. Parry, C. A. Gilligan, Testing stochastic software using pseudo-oracles, in: Proceedings of the 25th International Symposium on Software Testing and Analysis, ISSTA 2016, Saarbrücken, Germany, July 18-20, 2016, 2016, pp. 235-246. doi:10.1145/2931037.2931063.

URL http://doi.acm.org/10.1145/2931037.2931063

[214] M. J. Rutherford, A. Carzaniga, A. L. Wolf, Evaluating test suites and adequacy criteria using simulation-based models of distributed systems, IEEE Trans. Software Eng. 34 (4) (2008) 452-470. doi:10.1109/TSE. 2008.33 .

URL https://doi.org/10.1109/TSE.2008.33

[215] B. J. M. Grün, D. Schuler, A. Zeller, The impact of equivalent mutants, in: Second International Conference on Software Testing Verification and Validation, ICST 2009, Denver, Colorado, USA, April 1-4, 2009, Workshops Proceedings, 2009, pp. 192-199. doi:10.1109/ICSTW.2009.37.

URL http://dx.doi.org/10.1109/ICSTW.2009.37

[216] D. Schuler, V. Dallmeier, A. Zeller, Efficient mutation testing by checking invariant violations, in: Proceedings of the Eighteenth International Symposium on Software Testing and Analysis, ISSTA 2009, Chicago, IL, USA, 
July 19-23, 2009, 2009, pp. 69-80. doi:10.1145/1572272.1572282.

URL http://doi .acm.org/10.1145/1572272.1572282

[217] D. Schuler, A. Zeller, (un-)covering equivalent mutants, in: Third International Conference on Software Testing, Verification and Validation, ICST 2010, Paris, France, April 7-9, 2010, 2010, pp. 45-54. doi: 10.1109/ICST . 2010.30.

URL http://dx.doi.org/10.1109/ICST .2010.30

[218] D. Schuler, A. Zeller, Covering and uncovering equivalent mutants, Softw. Test., Verif. Reliab. 23 (5) (2013) 353-374. doi:10.1002/stvr.1473.

URL http://dx.doi.org/10.1002/stvr.1473

[219] B. Schwarz, D. Schuler, A. Zeller, Breeding high-impact mutations, in: Fourth IEEE International Conference on Software Testing, Verification and Validation, ICST 2012, Berlin, Germany, 21-25 March, 2011, Workshop Proceedings, 2011, pp. 382-387. doi:10.1109/ICSTW.2011.56. URL https://doi .org/10.1109/ICSTW .2011.56

[220] M. Papadakis, Y. L. Traon, Mutation testing strategies using mutant classification, in: Proceedings of the 28th Annual ACM Symposium on Applied Computing, SAC '13, Coimbra, Portugal, March 18-22, 2013, 2013, pp. 1223-1229. doi:10.1145/2480362.2480592.

URL http://doi.acm.org/10.1145/2480362.2480592

[221] M. Papadakis, M. E. Delamaro, Y. L. Traon, Mitigating the effects of equivalent mutants with mutant classification strategies, Sci. Comput. Program. 95 (2014) 298-319. doi:10.1016/j.scico.2014.05.012.

URL http://dx.doi.org/10.1016/j.scico.2014.05.012

[222] M. Kintis, M. Papadakis, N. Malevris, Isolating first order equivalent mutants via second order mutation, in: Fifth IEEE International Conference on Software Testing, Verification and Validation, ICST 2012, Montreal, QC, Canada, April 17-21, 2012, 2012, pp. 701-710. doi: 
10.1109/ICST.2012.160.

URL http://dx.doi.org/10.1109/ICST.2012.160

[223] M. Kintis, M. Papadakis, N. Malevris, Employing second-order mutation for isolating first-order equivalent mutants, Softw. Test., Verif. Reliab. 25 (5-7) (2015) 508-535. doi:10.1002/stvr.1529

URL http://dx.doi.org/10.1002/stvr.1529

[224] M. P. Usaola, P. R. Mateo, B. P. Lamancha, Reduction of test suites using mutation, in: Fundamental Approaches to Software Engineering - 15th International Conference, FASE 2012, Held as Part of the European Joint Conferences on Theory and Practice of Software, ETAPS 2012, Tallinn, Estonia, March 24 - April 1, 2012. Proceedings, 2012, pp. 425-438. doi: 10.1007/978-3-642-28872-2_29. URL http: //dx.doi.org/10.1007/978-3-642-28872-2_29

[225] D. Hao, L. Zhang, X. Wu, H. Mei, G. Rothermel, On-demand test suite reduction, in: 34th International Conference on Software Engineering, ICSE 2012, June 2-9, 2012, Zurich, Switzerland, 2012, pp. 738-748. doi: 10.1109/ICSE. 2012.6227144.

URL http://dx.doi.org/10.1109/ICSE.2012.6227144

[226] M. A. Alipour, A. Shi, R. Gopinath, D. Marinov, A. Groce, Evaluating non-adequate test-case reduction, in: Proceedings of the 31st IEEE/ACM International Conference on Automated Software Engineering, ASE 2016, Singapore, September 3-7, 2016, 2016, pp. 16-26. doi:10.1145/2970276. 2970361 .

URL http://doi .acm.org/10.1145/2970276.2970361

[227] D. C. Nguyen, A. Marchetto, P. Tonella, Change sensitivity based prioritization for audit testing of webservice compositions, in: Fourth IEEE International Conference on Software Testing, Verification and Validation, ICST 2012, Berlin, Germany, 21-25 March, 2011, Workshop Proceedings, 
2011, pp. 357-365. doi:10.1109/ICSTW.2011.50.

URL http://dx.doi.org/10.1109/ICSTW.2011.50

[228] D. Tengeri, L. Vidács, Á. Beszédes, J. Jász, G. Balogh, B. Vancsics, T. Gyimóthy, Relating code coverage, mutation score and test suite reducibility to defect density, in: Ninth IEEE International Conference on Software Testing, Verification and Validation Workshops, ICST Workshops 2016, Chicago, IL, USA, April 11-15, 2016, 2016, pp. 174-179. doi:10.1109/ICSTW.2016.25.

URL http://dx.doi.org/10.1109/ICSTW.2016.25

[229] B. Kurtz, P. Ammann, J. Offutt, M. Kurtz, Are we there yet? how redundant and equivalent mutants affect determination of test completeness, in: Ninth IEEE International Conference on Software Testing, Verification and Validation Workshops, ICST Workshops 2016, Chicago, IL, USA, April 11-15, 2016, 2016, pp. 142-151. doi:10.1109/ICSTW.2016.41.

URL https://doi.org/10.1109/ICSTW.2016.41

[230] G. Fraser, A. Zeller, Generating parameterized unit tests, in: Proceedings of the 20th International Symposium on Software Testing and Analysis, ISSTA 2011, Toronto, ON, Canada, July 17-21, 2011, 2011, pp. 364-374. doi:10.1145/2001420.2001464. URL http://doi . acm . org/10.1145/2001420.2001464

[231] T. Knauth, C. Fetzer, P. Felber, Assertion-driven development: Assessing the quality of contracts using meta-mutations, in: Second International Conference on Software Testing Verification and Validation, ICST 2009, Denver, Colorado, USA, April 1-4, 2009, Workshops Proceedings, 2009, pp. 182-191. doi:10.1109/ICSTW.2009.40.

URL https://doi .org/10.1109/ICSTW.2009.40

[232] G. Fraser, A. Arcuri, Evosuite: automatic test suite generation for object-oriented software, in: SIGSOFT/FSE'11 19th ACM SIGSOFT Symposium on the Foundations of Software Engineering (FSE-19) and 
ESEC'11: 13th European Software Engineering Conference (ESEC-13), Szeged, Hungary, September 5-9, 2011, 2011, pp. 416-419. doi:10.1145/ 2025113.2025179 .

URL http://doi.acm.org/10.1145/2025113.2025179

[233] H. Yoshida, S. Tokumoto, M. R. Prasad, I. Ghosh, T. Uehara, FSX: finegrained incremental unit test generation for $\mathrm{C} / \mathrm{C}++$ programs, in: Proceedings of the 25th International Symposium on Software Testing and Analysis, ISSTA 2016, Saarbrücken, Germany, July 18-20, 2016, 2016, pp. 106-117. doi:10.1145/2931037.2931055.

URL http://doi .acm.org/10.1145/2931037.2931055

[234] H. Yoshida, S. Tokumoto, M. R. Prasad, I. Ghosh, T. Uehara, FSX: a tool for fine-grained incremental unit test generation for $\mathrm{C} / \mathrm{C}++$ programs, in: Proceedings of the 24th ACM SIGSOFT International Symposium on Foundations of Software Engineering, FSE 2016, Seattle, WA, USA, November 13-18, 2016, 2016, pp. 1052-1056. doi:10.1145/2950290. 2983937.

URL http://doi.acm.org/10.1145/2950290.2983937

[235] S. Mirshokraie, A. Mesbah, K. Pattabiraman, PYTHIA: generating test cases with oracles for javascript applications, in: 2013 28th IEEE/ACM International Conference on Automated Software Engineering, ASE 2013, Silicon Valley, CA, USA, November 11-15, 2013, 2013, pp. 610-615. doi: 10.1109/ASE. 2013.6693121

URL http://dx.doi.org/10.1109/ASE.2013.6693121

[236] S. Mirshokraie, A. Mesbah, K. Pattabiraman, JSEFT: automated javascript unit test generation, in: 8th IEEE International Conference on Software Testing, Verification and Validation, ICST 2015, Graz, Austria, April 13-17, 2015, 2015, pp. 1-10. doi:10.1109/ICST.2015.7102595.

URL http://dx.doi .org/10.1109/ICST . 2015.7102595 
[237] M. Staats, G. Gay, M. P. E. Heimdahl, Automated oracle creation support, or: How I learned to stop worrying about fault propagation and love mutation testing, in: 34th International Conference on Software Engineering, ICSE 2012, June 2-9, 2012, Zurich, Switzerland, 2012, pp. 870-880. doi:10.1109/ICSE.2012.6227132

URL http://dx.doi.org/10.1109/ICSE.2012.6227132

[238] G. Gay, M. Staats, M. W. Whalen, M. P. E. Heimdahl, Automated oracle data selection support, IEEE Trans. Software Eng. 41 (11) (2015) 11191137. doi:10.1109/TSE.2015.2436920.

URL https://doi.org/10.1109/TSE.2015.2436920

[239] G. Jahangirova, D. Clark, M. Harman, P. Tonella, Test oracle assessment and improvement, in: Proceedings of the 25th International Symposium on Software Testing and Analysis, ISSTA 2016, Saarbrücken, Germany, July 18-20, 2016, 2016, pp. 247-258. doi:10.1145/2931037.2931062. URL http://doi .acm .org/10.1145/2931037.2931062

[240] E. T. Barr, M. Harman, P. McMinn, M. Shahbaz, S. Yoo, The oracle problem in software testing: A survey, IEEE Trans. Software Eng. 41 (5) (2015) 507-525. doi:10.1109/TSE.2014.2372785.

URL https://doi.org/10.1109/TSE.2014.2372785

[241] M. Papadakis, Y. L. Traon, Using mutants to locate "unknown" faults, in: Fifth IEEE International Conference on Software Testing, Verification and Validation, ICST 2012, Montreal, QC, Canada, April 17-21, 2012, 2012, pp. 691-700. doi:10.1109/ICST.2012.159.

URL http://dx.doi.org/10.1109/ICST.2012.159

[242] T. T. Chekam, M. Papadakis, Y. L. Traon, Assessing and comparing mutation-based fault localization techniques, CoRR abs/1607.05512.

URL http://arxiv.org/abs/1607.05512

[243] S. Moon, Y. Kim, M. Kim, S. Yoo, Ask the mutants: Mutating faulty programs for fault localization, in: Seventh IEEE International Conference 
on Software Testing, Verification and Validation, ICST 2014, March 31 2014-April 4, 2014, Cleveland, Ohio, USA, 2014, pp. 153-162. doi:10. 1109/ICST.2014.28

URL https://doi.org/10.1109/ICST .2014.28

[244] L. Zhang, L. Zhang, S. Khurshid, Injecting mechanical faults to localize developer faults for evolving software, in: Proceedings of the 2013 ACM SIGPLAN International Conference on Object Oriented Programming Systems Languages \& Applications, OOPSLA 2013, part of SPLASH 2013, Indianapolis, IN, USA, October 26-31, 2013, 2013, pp. 765-784. doi:10.1145/2509136.2509551. URL http://doi .acm.org/10.1145/2509136.2509551

[245] S. Hong, B. Lee, T. Kwak, Y. Jeon, B. Ko, Y. Kim, M. Kim, Mutationbased fault localization for real-world multilingual programs $(\mathrm{T})$, in: 30th IEEE/ACM International Conference on Automated Software Engineering, ASE 2015, Lincoln, NE, USA, November 9-13, 2015, 2015, pp. 464475. doi:10.1109/ASE.2015.14.

URL http://dx.doi .org/10.1109/ASE.2015.14

[246] S. Hong, T. Kwak, B. Lee, Y. Jeon, B. Ko, Y. Kim, M. Kim, MUSEUM: debugging real-world multilingual programs using mutation analysis, Information \& Software Technology 82 (2017) 80-95. doi:10.1016/ j.infsof.2016.10.002. URL https://doi.org/10.1016/j.inf sof .2016.10.002

[247] S. S. Murtaza, N. H. Madhavji, M. Gittens, Z. Li, Diagnosing new faults using mutants and prior faults, in: Proceedings of the 33rd International Conference on Software Engineering, ICSE 2011, Waikiki, Honolulu , HI, USA, May 21-28, 2011, 2011, pp. 960-963. doi:10.1145/1985793. 1985959.

URL http://doi.acm.org/10.1145/1985793.1985959 
[248] S. S. Murtaza, A. Hamou-Lhadj, N. H. Madhavji, M. Gittens, An empirical study on the use of mutant traces for diagnosis of faults in deployed systems, Journal of Systems and Software 90 (2014) 29-44. doi:10.1016/j.jss.2013.11.1094

URL https://doi.org/10.1016/j.jss.2013.11.1094

[249] V. Musco, M. Monperrus, P. Preux, Mutation-based graph inference for fault localization, in: 16th IEEE International Working Conference on Source Code Analysis and Manipulation, SCAM 2016, Raleigh, NC, USA, October 2-3, 2016, 2016, pp. 97-106. doi:10.1109/SCAM.2016.24.

URL http://dx.doi.org/10.1109/SCAM.2016.24

[250] V. Debroy, W. E. Wong, Using mutation to automatically suggest fixes for faulty programs, in: Third International Conference on Software Testing, Verification and Validation, ICST 2010, Paris, France, April 7-9, 2010, 2010, pp. 65-74. doi:10.1109/ICST.2010.66.

URL http://dx.doi.org/10.1109/ICST.2010.66

[251] V. Debroy, W. E. Wong, Combining mutation and fault localization for automated program debugging, Journal of Systems and Software 90 (2014) 45-60. doi:10.1016/j.jss.2013.10.042

URL https://doi.org/10.1016/j.jss.2013.10.042

[252] W. Weimer, T. Nguyen, C. Le Goues, S. Forrest, Automatically finding patches using genetic programming, in: 31st International Conference on Software Engineering, ICSE 2009, May 16-24, 2009, Vancouver, Canada, Proceedings, 2009, pp. 364-374. doi:10.1109/ICSE.2009.5070536.

URL http://dx.doi .org/10.1109/ICSE.2009.5070536

[253] C. Le Goues, M. Dewey-Vogt, S. Forrest, W. Weimer, A systematic study of automated program repair: Fixing 55 out of 105 bugs for $\$ 8$ each, in: 34th International Conference on Software Engineering, ICSE 2012, June 2-9, 2012, Zurich, Switzerland, 2012, pp. 3-13. doi:10.1109/ICSE. 2012. 
6227211.

URL http://dx.doi .org/10.1109/ICSE.2012.6227211

[254] W. Weimer, Z. P. Fry, S. Forrest, Leveraging program equivalence for adaptive program repair: Models and first results, in: 2013 28th IEEE/ACM International Conference on Automated Software Engineering, ASE 2013, Silicon Valley, CA, USA, November 11-15, 2013, 2013, pp. 356-366. doi:10.1109/ASE.2013.6693094 URL http://dx.doi.org/10.1109/ASE.2013.6693094

[255] S. H. Tan, A. Roychoudhury, relifix: Automated repair of software regressions, in: 37th IEEE/ACM International Conference on Software Engineering, ICSE 2015, Florence, Italy, May 16-24, 2015, Volume 1, 2015, pp. 471-482. doi:10.1109/ICSE.2015.65.

URL http://dx.doi.org/10.1109/ICSE.2015.65

[256] F. Long, M. Rinard, Staged program repair with condition synthesis, in: Proceedings of the 2015 10th Joint Meeting on Foundations of Software Engineering, ESEC/FSE 2015, Bergamo, Italy, August 30 - September 4, 2015, 2015, pp. 166-178. doi:10.1145/2786805.2786811.

URL http://doi . acm . org/10.1145/2786805.2786811

[257] D. Kim, J. Nam, J. Song, S. Kim, Automatic patch generation learned from human-written patches, in: 35th International Conference on Software Engineering, ICSE '13, San Francisco, CA, USA, May 18-26, 2013, 2013, pp. 802-811. doi:10.1109/ICSE.2013.6606626.

URL https://doi .org/10.1109/ICSE.2013.6606626

[258] J. Petke, S. Haraldsson, M. Harman, w. langdon, D. White, J. Woodward, Genetic improvement of software: a comprehensive survey, IEEE Transactions on Evolutionary Computation PP (99) (2017) 1-1. doi: 10.1109/TEVC.2017.2693219.

[259] S. Chandra, E. Torlak, S. Barman, R. Bodík, Angelic debugging, in: Proceedings of the 33rd International Conference on Software Engineering, 
ICSE 2011, Waikiki, Honolulu , HI, USA, May 21-28, 2011, 2011, pp. 121-130. doi:10.1145/1985793.1985811.

URL http://doi .acm.org/10.1145/1985793.1985811

[260] J. Zhang, Z. Wang, L. Zhang, D. Hao, L. Zang, S. Cheng, L. Zhang, Predictive mutation testing, in: Proceedings of the 25th International Symposium on Software Testing and Analysis, ISSTA 2016, Saarbrücken, Germany, July 18-20, 2016, 2016, pp. 342-353. doi:10.1145/2931037. 2931038.

URL http://doi .acm.org/10.1145/2931037.2931038

[261] J. M. Rojas, G. Fraser, Code defenders: A mutation testing game, in: Ninth IEEE International Conference on Software Testing, Verification and Validation Workshops, ICST Workshops 2016, Chicago, IL, USA, April 11-15, 2016, 2016, pp. 162-167. doi:10.1109/ICSTW.2016.43. URL https://doi.org/10.1109/ICSTW .2016.43

[262] J. M. Rojas, T. D. White, B. S. Clegg, G. Fraser, Code defenders: crowdsourcing effective tests and subtle mutants with a mutation testing game in: Proceedings of the 39th International Conference on Software Engineering, ICSE 2017, Buenos Aires, Argentina, May 20-28, 2017, 2017, pp. 677-688.

URL http: //dl . acm.org/citation . cfm?id=3097449

[263] M. Harman, Y. Jia, W. B. Langdon, A manifesto for higher order mutation testing, in: Third International Conference on Software Testing, Verification and Validation, ICST 2010, Paris, France, April 7-9, 2010, Workshops Proceedings, 2010, pp. 80-89. doi:10.1109/ICSTW.2010.13. URL https://doi.org/10.1109/ICSTW.2010.13

[264] Y. Jia, M. Harman, Constructing subtle faults using higher order mutation testing, in: Eighth IEEE International Working Conference on Source Code Analysis and Manipulation (SCAM 2008), 28-29 September 2008, 
Beijing, China, 2008, pp. 249-258. doi:10.1109/SCAM.2008.36.

URL https://doi.org/10.1109/SCAM.2008.36

[265] M. Harman, Y. Jia, P. R. Mateo, M. Polo, Angels and monsters: an empirical investigation of potential test effectiveness and efficiency improvement from strongly subsuming higher order mutation, in: ACM/IEEE International Conference on Automated Software Engineering, ASE '14, Vasteras, Sweden - September 15 - 19, 2014, 2014, pp. 397-408. doi: $10.1145 / 2642937.2643008$

URL http://doi .acm.org/10.1145/2642937.2643008

[266] E. Omar, S. Ghosh, D. Whitley, Constructing subtle higher order mutants for java and aspectj programs, in: IEEE 24th International Symposium on Software Reliability Engineering, ISSRE 2013, Pasadena, CA, USA, November 4-7, 2013, 2013, pp. 340-349. doi:10.1109/ISSRE.2013. 6698887.

URL https://doi.org/10.1109/ISSRE.2013.6698887

[267] E. Omar, S. Ghosh, D. Whitley, Subtle higher order mutants, Information \& Software Technology 81 (2017) 3-18. doi:10.1016/j.infsof.2016. 01.016 .

URL https://doi.org/10.1016/j.infsof .2016.01.016

[268] E. Omar, S. Ghosh, D. Whitley, Comparing search techniques for finding subtle higher order mutants, in: Genetic and Evolutionary Computation Conference, GECCO '14, Vancouver, BC, Canada, July 12-16, 2014, 2014, pp. 1271-1278. doi:10.1145/2576768.2598286.

URL http://doi .acm.org/10.1145/2576768.2598286

[269] F. Wu, M. Harman, Y. Jia, J. Krinke, HOMI: searching higher order mutants for software improvement, in: Search Based Software Engineering - 8th International Symposium, SSBSE 2016, Raleigh, NC, USA, October 8-10, 2016, Proceedings, 2016, pp. 18-33. doi:10.1007/ 
978-3-319-47106-8_2.

URL http://dx.doi .org/10.1007/978-3-319-47106-8_2

[270] D. Shin, S. Yoo, D. Bae, Diversity-aware mutation adequacy criterion for improving fault detection capability, in: Ninth IEEE International Conference on Software Testing, Verification and Validation Workshops, ICST Workshops 2016, Chicago, IL, USA, April 11-15, 2016, 2016, pp. 122-131. doi:10.1109/ICSTW.2016.37.

URL https : //doi .org/10.1109/ICSTW .2016.37

[271] D. Shin, S. Yoo, D. H. Bae, A theoretical and empirical study of diversityaware mutation adequacy criterion, IEEE Transactions on Software Engineering PP (99) (2017) 1-1. doi:10.1109/TSE.2017.2732347.

[272] C. Henard, M. Papadakis, G. Perrouin, J. Klein, Y. L. Traon, Assessing software product line testing via model-based mutation: An application to similarity testing, in: Sixth IEEE International Conference on Software Testing, Verification and Validation, ICST 2013 Workshops Proceedings, Luxembourg, Luxembourg, March 18-22, 2013, 2013, pp. 188-197. doi: 10.1109/ICSTW. 2013.30.

URL http://dx.doi .org/10.1109/ICSTW.2013.30

[273] C. Henard, M. Papadakis, Y. L. Traon, Mutalog: A tool for mutating logic formulas, in: Seventh IEEE International Conference on Software Testing, Verification and Validation, ICST 2014 Workshops Proceedings, March 31 - April 4, 2014, Cleveland, Ohio, USA, 2014, pp. 399-404. doi: 10.1109/ICSTW. 2014.54.

URL https : //doi.org/10.1109/ICSTW . 2014.54

[274] C. Henard, M. Papadakis, G. Perrouin, J. Klein, Y. L. Traon, Towards automated testing and fixing of re-engineered feature models, in: 35th International Conference on Software Engineering, ICSE '13, San Francisco, CA, USA, May 18-26, 2013, 2013, pp. 1245-1248. doi:10.1109/ICSE. 
2013.6606689

URL https://doi.org/10.1109/ICSE.2013.6606689

[275] C. Henard, M. Papadakis, Y. L. Traon, Mutation-based generation of software product line test configurations, in: Search-Based Software Engineering - 6th International Symposium, SSBSE 2014, Fortaleza, Brazil, August 26-29, 2014. Proceedings, 2014, pp. 92-106. doi:10.1007/ 978-3-319-09940-8_7 URL http://dx.doi.org/10.1007/978-3-319-09940-8_7

[276] R. A. M. Filho, S. R. Vergilio, A mutation and multi-objective test data generation approach for feature testing of software product lines in: 29th Brazilian Symposium on Software Engineering, SBES 2015, Belo Horizonte, MG, Brazil, September 21-26, 2015, 2015, pp. 21-30. doi:10.1109/SBES.2015.17

URL https://doi.org/10.1109/SBES.2015.17

[277] R. A. M. Filho, S. R. Vergilio, A multi-objective test data generation approach for mutation testing of feature models, J. Software Eng. R\&D 4 (2016) 4. doi:10.1186/s40411-016-0030-9

URL https://doi.org/10.1186/s40411-016-0030-9

[278] P. Arcaini, A. Gargantini, P. Vavassori, Generating tests for detecting faults in feature models, in: 8th IEEE International Conference on Software Testing, Verification and Validation, ICST 2015, Graz, Austria, April 13-17, 2015, 2015, pp. 1-10. doi:10.1109/ICST.2015.7102591.

URL http://dx.doi.org/10.1109/ICST . 2015.7102591

[279] P. Arcaini, A. Gargantini, P. Vavassori, Automatic detection and removal of conformance faults in feature models, in: 2016 IEEE International Conference on Software Testing, Verification and Validation, ICST 2016, Chicago, IL, USA, April 11-15, 2016, 2016, pp. 102-112. doi:10.1109/ICST.2016.10

URL http://dx.doi.org/10.1109/ICST.2016.10 
[280] M. B. Trakhtenbrot, Implementation-oriented mutation testing of statechart models, in: Third International Conference on Software Testing, Verification and Validation, ICST 2010, Paris, France, April 7-9, 2010, Workshops Proceedings, 2010, pp. 120-125. doi:10.1109/ICSTW.2010. 55.

URL http://dx.doi.org/10.1109/ICSTW.2010.55

[281] B. K. Aichernig, E. Jöbstl, Towards symbolic model-based mutation testing: Pitfalls in expressing semantics as constraints, in: Fifth IEEE International Conference on Software Testing, Verification and Validation, ICST 2012, Montreal, QC, Canada, April 17-21, 2012, 2012, pp. 752-757. doi:10.1109/ICST.2012.169.

URL http://dx.doi.org/10.1109/ICST.2012.169

[282] B. K. Aichernig, H. Brandl, E. Jöbstl, W. Krenn, Efficient mutation killers in action, in: Fourth IEEE International Conference on Software Testing, Verification and Validation, ICST 2011, Berlin, Germany, March 21-25, 2011, 2011, pp. 120-129. doi:10.1109/ICST.2011.57.

URL http://dx.doi.org/10.1109/ICST .2011.57

[283] B. K. Aichernig, H. Brandl, E. Jöbstl, W. Krenn, R. Schlick, S. Tiran, Killing strategies for model-based mutation testing, Softw. Test., Verif. Reliab. 25 (8) (2015) 716-748. doi:10.1002/stvr.1522

URL http://dx.doi.org/10.1002/stvr.1522

[284] F. Belli, M. Beyazit, Exploiting model morphology for event-based testing, IEEE Trans. Software Eng. 41 (2) (2015) 113-134. doi:10.1109/TSE. 2014.2360690 .

URL https://doi .org/10.1109/TSE.2014.2360690

[285] K. El-Fakih, A. Kolomeez, S. Prokopenko, N. Yevtushenko, Extended finite state machine based test derivation driven by user defined faults, in: First International Conference on Software Testing, Verification, and Validation, ICST 2008, Lillehammer, Norway, April 9-11, 2008, 2008, pp. 
308-317. doi:10.1109/ICST.2008.16.

URL http://dx.doi.org/10.1109/ICST.2008.16

[286] T. Su, G. Meng, Y. Chen, K. Wu, W. Yang, Y. Yao, G. Pu, Y. Liu, Z. Su, Guided, stochastic model-based gui testing of android apps, in: Proceedings of the 2017 11th Joint Meeting on Foundations of Software Engineering, ESEC/FSE 2017, ACM, New York, NY, USA, 2017, pp. 245-256. doi:10.1145/3106237.3106298. URL http://doi .acm .org/10.1145/3106237.3106298

[287] B. K. Aichernig, S. Marcovic, R. Schumi, Property-based testing with external test-case generators, in: 2017 IEEE International Conference on Software Testing, Verification and Validation Workshops (ICSTW), 2017, pp. 337-346. doi:10.1109/ICSTW.2017.62.

[288] B. K. Aichernig, F. Lorber, Towards generation of adaptive test cases from partial models of determinized timed automata, in: Eighth IEEE International Conference on Software Testing, Verification and Validation, ICST 2015 Workshops, Graz, Austria, April 13-17, 2015, 2015, pp. 1-6. doi:10.1109/ICSTW.2015.7107409.

URL http://dx.doi .org/10.1109/ICSTW.2015.7107409

[289] B. K. Aichernig, F. Lorber, D. Nickovic, Time for mutants - model-based mutation testing with timed automata, in: Tests and Proofs - 7th International Conference, TAP 2013, Budapest, Hungary, June 16-20, 2013. Proceedings, 2013, pp. 20-38. doi:10.1007/978-3-642-38916-0_2. URL https : //doi .org/10.1007/978-3-642-38916-0_2

[290] K. G. Larsen, F. Lorber, B. Nielsen, U. M. Nyman, Mutation-based testcase generation with ecdar, in: 2017 IEEE International Conference on Software Testing, Verification and Validation Workshops (ICSTW), 2017, pp. 319-328. doi:10.1109/ICSTW.2017.60

[291] T. Zhou, H. Sun, J. Liu, X. Chen, D. Du, Improving testing coverage for safety-critical system by mutated specification, in: 21st Asia- 
Pacific Software Engineering Conference, APSEC 2014, Jeju, South Korea, December 1-4, 2014. Volume 1: Research Papers, 2014, pp. 43-46. doi:10.1109/APSEC.2014.15.

URL https://doi.org/10.1109/APSEC.2014.15

[292] S. F. Adra, P. McMinn, Mutation operators for agent-based models, in: Third International Conference on Software Testing, Verification and Validation, ICST 2010, Paris, France, April 7-9, 2010, Workshops Proceedings, 2010, pp. 151-156. doi:10.1109/ICSTW.2010.9. URL http://dx.doi.org/10.1109/ICSTW.2010.9

[293] M. Stephan, M. H. Alalfi, A. Stevenson, J. R. Cordy, Using mutation analysis for a model-clone detector comparison framework, in: 35th International Conference on Software Engineering, ICSE '13, San Francisco, CA, USA, May 18-26, 2013, 2013, pp. 1261-1264. doi:10.1109/ICSE. 2013.6606693 .

URL http://dx.doi.org/10.1109/ICSE.2013.6606693

[294] C. K. Roy, J. R. Cordy, A mutation/injection-based automatic framework for evaluating code clone detection tools, in: Second International Conference on Software Testing Verification and Validation, ICST 2009, Denver, Colorado, USA, April 1-4, 2009, Workshops Proceedings, 2009, pp. 157166. doi:10.1109/ICSTW.2009.18. URL http://dx.doi.org/10.1109/ICSTW.2009.18

[295] M. Stephan, M. H. Alalfi, J. R. Cordy, Towards a taxonomy for simulink model mutations, in: Seventh IEEE International Conference on Software Testing, Verification and Validation, ICST 2014 Workshops Proceedings, March 31 - April 4, 2014, Cleveland, Ohio, USA, 2014, pp. 206-215. doi: 10.1109/ICSTW.2014.17.

URL http://dx.doi.org/10.1109/ICSTW.2014.17

[296] I. Pill, I. Rubil, F. Wotawa, M. Nica, SIMULTATE: A toolset for fault injection and mutation testing of simulink models, in: Ninth IEEE In- 
ternational Conference on Software Testing, Verification and Validation Workshops, ICST Workshops 2016, Chicago, IL, USA, April 11-15, 2016, 2016, pp. 168-173. doi:10.1109/ICSTW.2016.21.

URL http://dx.doi.org/10.1109/ICSTW.2016.21

[297] Y. Khan, J. Hassine, Mutation operators for the atlas transformation language, in: Sixth IEEE International Conference on Software Testing, Verification and Validation, ICST 2013 Workshops Proceedings, Luxembourg, Luxembourg, March 18-22, 2013, 2013, pp. 43-52. doi: 10.1109/ICSTW.2013.13. URL http://dx.doi.org/10.1109/ICSTW.2013.13

[298] J. Troya, A. Bergmayr, L. Burgueño, M. Wimmer, Towards systematic mutations for and with ATL model transformations, in: Eighth IEEE International Conference on Software Testing, Verification and Validation, ICST 2015 Workshops, Graz, Austria, April 13-17, 2015, 2015, pp. 1-10. doi:10.1109/ICSTW.2015.7107455.

URL http://dx.doi.org/10.1109/ICSTW .2015.7107455

[299] M. Tisi, F. Jouault, P. Fraternali, S. Ceri, J. Bézivin, On the use of higherorder model transformations, in: Model Driven Architecture - Foundations and Applications: 5th European Conference, ECMDA-FA 2009, Enschede, The Netherlands, June 23-26, 2009. Proceedings, Vol. 5562 of LNCS, 2009, pp. 18-33. doi:10.1007/978-3-642-02674-4_3. URL http://dx .doi .org/10.1007/978-3-642-02674-4_3

[300] V. Aranega, J. Mottu, A. Etien, T. Degueule, B. Baudry, J. Dekeyser, Towards an automation of the mutation analysis dedicated to model transformation, Softw. Test., Verif. Reliab. 25 (5-7) (2015) 653-683. doi:10.1002/stvr.1532,

URL http://dx.doi.org/10.1002/stvr.1532

[301] A. Bartel, B. Baudry, F. Munoz, J. Klein, T. Mouelhi, Y. L. Traon, Model driven mutation applied to adaptative systems testing, in: Fourth IEEE 
International Conference on Software Testing, Verification and Validation, ICST 2011 Workshops Proceedings, March 21 - March 25, 2011, Berlin, Germany, 2011, pp. 408-413. doi:10.1109/ICSTW.2011.24.

URL http://dx.doi.org/10.1109/ICSTW.2011.24

[302] P. Arcaini, A. Gargantini, E. Riccobene, Using mutation to assess fault detection capability of model review, Softw. Test., Verif. Reliab. 25 (5-7) (2015) 629-652. doi:10.1002/stvr.1530. URL http://dx.doi.org/10.1002/stvr.1530

[303] M. Kaplan, T. Klinger, A. M. Paradkar, A. Sinha, C. Williams, C. Yilmaz, Less is more: A minimalistic approach to UML model-based conformance test generation, in: First International Conference on Software Testing, Verification, and Validation, ICST 2008, Lillehammer, Norway, April 911, 2008, 2008, pp. 82-91. doi:10.1109/ICST.2008.48.

URL http://dx.doi .org/10.1109/ICST .2008.48

[304] G. Fraser, F. Wotawa, Using model-checkers to generate and analyze property relevant test-cases, Software Quality Journal 16 (2) (2008) 161-183. doi:10.1007/s11219-007-9031-6

URL https://doi .org/10.1007/s11219-007-9031-6

[305] M. Trakhtenbrot, Mutation patterns for temporal requirements of reactive systems, in: 2017 IEEE International Conference on Software Testing, Verification and Validation Workshops (ICSTW), 2017, pp. 116-121. doi: 10.1109/ICSTW.2017.27.

[306] A. Sullivan, K. Wang, R. N. Zaeem, S. Khurshid, Automated test generation and mutation testing for alloy, in: 2017 IEEE International Conference on Software Testing, Verification and Validation (ICST), 2017, pp. 264-275. doi:10.1109/ICST.2017.31.

[307] D. Xu, O. el Ariss, W. Xu, L. Wang, Testing aspect-oriented programs with finite state machines, Softw. Test., Verif. Reliab. 22 (4) (2012) $267-$ 293. doi:10.1002/stvr.440 
[308] B. Lindström, S. F. Andler, J. Offutt, P. Pettersson, D. Sundmark, Mutating aspect-oriented models to test cross-cutting concerns, in: Eighth IEEE International Conference on Software Testing, Verification and Validation, ICST 2015 Workshops, Graz, Austria, April 13-17, 2015, 2015, pp. 1-10. doi:10.1109/ICSTW.2015.7107456

URL https ://doi .org/10.1109/ICSTW . 2015.7107456

[309] Y. Elrakaiby, T. Mouelhi, Y. L. Traon, Testing obligation policy enforcement using mutation analysis, in: Fifth IEEE International Conference on Software Testing, Verification and Validation, ICST 2012, Montreal, QC, Canada, April 17-21, 2012, 2012, pp. 673-680. doi: 10.1109/ICST.2012.157.

URL http://dx.doi.org/10.1109/ICST.2012.157

[310] T. Mouelhi, F. Fleurey, B. Baudry, A generic metamodel for security policies mutation, in: First International Conference on Software Testing Verification and Validation, ICST 2008, Lillehammer, Norway, April 9-11, 2008, Workshops Proceedings, 2008, pp. 278-286. doi:10.1109/ICSTW. 2008.2 .

URL http://dx.doi .org/10.1109/ICSTW.2008.2

[311] A. Bertolino, S. Daoudagh, F. Lonetti, E. Marchetti, F. Martinelli, P. Mori, Testing of polpa-based usage control systems, Software Quality Journal 22 (2) (2014) 241-271. doi:10.1007/s11219-013-9216-0. URL http://dx.doi.org/10.1007/s11219-013-9216-0

[312] T. Mouelhi, Y. L. Traon, B. Baudry, Transforming and selecting functional test cases for security policy testing, in: Second International Conference on Software Testing Verification and Validation, ICST 2009, Denver, Colorado, USA, April 1-4, 2009, 2009, pp. 171-180. doi:10.1109/ICST. 2009.49 .

URL http://dx.doi.org/10.1109/ICST .2009.49 
[313] P. H. Nguyen, M. Papadakis, I. Rubab, Testing delegation policy enforcement via mutation analysis, in: Sixth IEEE International Conference on Software Testing, Verification and Validation, ICST 2013 Workshops Proceedings, Luxembourg, Luxembourg, March 18-22, 2013, 2013, pp. 34-42. doi:10.1109/ICSTW.2013.12.

URL http://dx.doi.org/10.1109/ICSTW.2013.12

[314] J. Hwang, T. Xie, D. E. Kateb, T. Mouelhi, Y. L. Traon, Selection of regression system tests for security policy evolution, in: IEEE/ACM International Conference on Automated Software Engineering, ASE'12, Essen, Germany, September 3-7, 2012, 2012, pp. 266-269. doi:10.1145/ 2351676.2351719 .

URL http://doi .acm.org/10.1145/2351676.2351719

[315] F. Dadeau, P. Héam, R. Kheddam, Mutation-based test generation from security protocols in HLPSL, in: Fourth IEEE International Conference on Software Testing, Verification and Validation, ICST 2011, Berlin, Germany, March 21-25, 2011, 2011, pp. 240-248. doi:10.1109/ICST.2011. 42 .

URL http://dx.doi.org/10.1109/ICST.2011.42

[316] F. Dadeau, P. Héam, R. Kheddam, G. Maatoug, M. Rusinowitch, Modelbased mutation testing from security protocols in HLPSL, Softw. Test., Verif. Reliab. 25 (5-7) (2015) 684-711. doi:10.1002/stvr.1531.

URL http://dx.doi.org/10.1002/stvr.1531

[317] M. Papadakis, C. Henard, Y. L. Traon, Sampling program inputs with mutation analysis: Going beyond combinatorial interaction testing, in: Seventh IEEE International Conference on Software Testing, Verification and Validation, ICST 2014, March 31 2014-April 4, 2014, Cleveland, Ohio, USA, 2014, pp. 1-10. doi:10.1109/ICST.2014.11.

URL http://dx.doi.org/10.1109/ICST.2014.11 
[318] Z. Zhang, T. Wu, J. Zhang, Boundary value analysis in automatic whitebox test generation, in: 26th IEEE International Symposium on Software Reliability Engineering, ISSRE 2015, Gaithersbury, MD, USA, November 2-5, 2015, 2015, pp. 239-249. doi:10.1109/ISSRE. 2015.7381817.

URL http://dx.doi.org/10.1109/ISSRE.2015.7381817

[319] M. Patrick, Y. Jia, Kernel density adaptive random testing, in: Eighth IEEE International Conference on Software Testing, Verification and Validation, ICST 2015 Workshops, Graz, Austria, April 13-17, 2015, 2015, pp. 1-10. doi:10.1109/ICSTW.2015.7107451. URL http://dx.doi.org/10.1109/ICSTW.2015.7107451

[320] M. Patrick, Y. Jia, KD-ART: should we intensify or diversify tests to kill mutants?, Information \& Software Technology 81 (2017) 36-51. doi: $10.1016 / j$.infsof.2016.04.009

URL https://doi.org/10.1016/j.infsof .2016.04.009

[321] J. P. Galeotti, C. A. Furia, E. May, G. Fraser, A. Zeller, Inferring loop invariants by mutation, dynamic analysis, and static checking, IEEE Trans. Software Eng. 41 (10) (2015) 1019-1037. doi:10.1109/TSE. 2015. 2431688 .

URL http://dx.doi.org/10.1109/TSE.2015.2431688

[322] C. Andrés, M. G. Merayo, M. Núñez, Passive testing of stochastic timed systems, in: Second International Conference on Software Testing Verification and Validation, ICST 2009, Denver, Colorado, USA, April 1-4, 2009, 2009, pp. 71-80. doi:10.1109/ICST.2009.35

URL http://dx.doi.org/10.1109/ICST .2009.35

[323] C. Andrés, M. G. Merayo, C. Molinero, Advantages of mutation in passive testing: An empirical study, in: Second International Conference on Software Testing Verification and Validation, ICST 2009, Denver, Colorado, USA, April 1-4, 2009, Workshops Proceedings, 2009, pp. 230-239. 
doi:10.1109/ICSTW.2009.33.

URL http://dx.doi.org/10.1109/ICSTW .2009.33

[324] C. Andrés, M. G. Merayo, M. Núñez, Formal passive testing of timed systems: theory and tools, Softw. Test., Verif. Reliab. 22 (6) (2012) 365405. doi:10.1002/stvr.1464

URL http://dx.doi.org/10.1002/stvr.1464

[325] T. Pankumhang, M. Rutherford, Iterative instrumentation for code coverage in time-sensitive systems, in: 8th IEEE International Conference on Software Testing, Verification and Validation, ICST 2015, Graz, Austria, April 13-17, 2015, 2015, pp. 1-10. doi:10.1109/ICST.2015.7102594.

URL http://dx.doi .org/10.1109/ICST .2015.7102594

[326] A. Groce, I. Ahmed, C. Jensen, P. E. McKenney, How verified is my code? falsification-driven verification (T), in: 30th IEEE/ACM International Conference on Automated Software Engineering, ASE 2015, Lincoln, NE, USA, November 9-13, 2015, 2015, pp. 737-748. doi: 10.1109/ASE. 2015.40.

URL http://dx .doi .org/10.1109/ASE .2015.40

[327] J. Svajlenko, C. K. Roy, S. Duszynski, Forksim: Generating software forks for evaluating cross-project similarity analysis tools, in: 13th IEEE International Working Conference on Source Code Analysis and Manipulation, SCAM 2013, Eindhoven, Netherlands, September 22-23, 2013, 2013, pp. 37-42. doi:10.1109/SCAM. 2013.6648182.

URL http://dx .doi .org/10.1109/SCAM.2013.6648182

[328] D. Bowes, T. Hall, M. Harman, Y. Jia, F. Sarro, F. Wu, Mutation-aware fault prediction, in: Proceedings of the 25th International Symposium on Software Testing and Analysis, ISSTA 2016, Saarbrücken, Germany, July 18-20, 2016, 2016, pp. 330-341. doi:10.1145/2931037.2931039

URL http://doi .acm .org/10.1145/2931037.2931039 
[329] J. Zhang, Y. Lou, L. Zhang, D. Hao, L. Zhang, H. Mei, Isomorphic regression testing: executing uncovered branches without test augmentation, in: Proceedings of the 24th ACM SIGSOFT International Symposium on Foundations of Software Engineering, FSE 2016, Seattle, WA, USA, November 13-18, 2016, 2016, pp. 883-894. doi:10.1145/2950290. 2950313.

URL http://doi .acm.org/10.1145/2950290.2950313

[330] D. D. Nardo, F. Pastore, L. C. Briand, Generating complex and faulty test data through model-based mutation analysis, in: 8th IEEE International Conference on Software Testing, Verification and Validation, ICST 2015, Graz, Austria, April 13-17, 2015, 2015, pp. 1-10. doi:10.1109/ICST. 2015.7102589 .

URL http://dx.doi.org/10.1109/ICST . 2015.7102589

[331] P. Arcaini, A. Gargantini, E. Riccobene, P. Vavassori, Rehabilitating equivalent mutants as static anomaly detectors in software artifacts, in: Eighth IEEE International Conference on Software Testing, Verification and Validation, ICST 2015 Workshops, Graz, Austria, April 13-17, 2015, 2015, pp. 1-6. doi:10.1109/ICSTW.2015.7107452

URL https://doi .org/10.1109/ICSTW . 2015.7107452

[332] P. Arcaini, A. Gargantini, E. Riccobene, P. Vavassori, A novel use of equivalent mutants for static anomaly detection in software artifacts, Information \& Software Technology 81 (2017) 52-64. doi:10.1016/j.infsof. 2016.01 .019

URL https://doi.org/10.1016/j.infsof .2016.01.019

[333] B. Baudry, S. Allier, M. Monperrus, Tailored source code transformations to synthesize computationally diverse program variants, in: International Symposium on Software Testing and Analysis, ISSTA '14, San Jose, CA, USA - July 21 - 26, 2014, 2014, pp. 149-159. doi:10.1145/2610384. 
2610415.

URL http://doi.acm.org/10.1145/2610384.2610415

[334] B. Lisper, B. Lindstrm, P. Potena, M. Saadatmand, M. Bohlin, Targeted mutation: Efficient mutation analysis for testing non-functional properties, in: 2017 IEEE International Conference on Software Testing, Verification and Validation Workshops (ICSTW), 2017, pp. 65-68. doi:10.1109/ICSTW.2017.18,

[335] A. Babu, mutatepy: A mutation testing tool for c, "Last Accessed May $2017 "$

URL "http://members.femto-st.fr/pierre-cyrille-heam/ mutatepy"

[336] I. Moore, Jester and pester, "Last Accessed May 2017" (2001). URL "http://jester.sourceforge.net/"

[337] M. E. Delamaro, J. C. Maldonado, A. M. R. Vincenzi, Proteum/IM 2.0: An Integrated Mutation Testing Environment, Springer US, Boston, MA, 2001, Ch. Mutation Testing for the New Century, pp. 91-101. doi:10. 1007/978-1-4757-5939-6_17. URL http://dx.doi.org/10.1007/978-1-4757-5939-6_17

[338] J. H. Andrews, Y. Zhang, General test result checking with log file analysis, IEEE Trans. Software Eng. 29 (7) (2003) 634-648. doi:10.1109/ TSE.2003.1214327.

URL https://doi .org/10.1109/TSE.2003.1214327

[339] J. H. Andrews, L. C. Briand, Y. Labiche, Is mutation an appropriate tool for testing experiments?, in: G. Roman, W. G. Griswold, B. Nuseibeh (Eds.), 27th International Conference on Software Engineering (ICSE 2005), 15-21 May 2005, St. Louis, Missouri, USA, ACM, 2005, pp. 402411. doi:10.1145/1062455.1062530.

URL http://doi .acm.org/10.1145/1062455.1062530 
[340] Y. Ma, J. Offutt, Y. R. Kwon, Mujava: a mutation system for java, in: L. J. Osterweil, H. D. Rombach, M. L. Soffa (Eds.), 28th International Conference on Software Engineering (ICSE 2006), Shanghai, China, May 20-28, 2006, ACM, 2006, pp. 827-830. doi:10.1145/1134425

URL http://doi .acm.org/10.1145/1134425

[341] J. Offutt, Y. Ma, Y. R. Kwon, An experimental mutation system for java, ACM SIGSOFT Software Engineering Notes 29 (5) (2004) 1-4. doi: 10.1145/1022494.1022537.

URL http://doi .acm.org/10.1145/1022494.1022537

[342] H. Do, G. Rothermel, On the use of mutation faults in empirical assessments of test case prioritization techniques, IEEE Trans. Software Eng. 32 (9) (2006) 733-752. doi:10.1109/TSE.2006.92.

URL https://doi.org/10.1109/TSE.2006.92

[343] J. Tuya, M. J. Suarez-Cabal, C. de la Riva, Sqlmutation: A tool to generate mutants of sql database queries, in: Second Workshop on Mutation Analysis (Mutation 2006 - ISSRE Workshops 2006), 2006, pp. 1-1. doi:10.1109/MUTATION.2006.13

[344] "jumble testing tool for java", "Last Accessed May 2017" (2007). URL "http://jumble.sourceforge.net/"

[345] X. Feng, S. Marr, T. O'Callaghan, Estp: An experimental software testing platform, in: Testing: Academic Industrial Conference - Practice and Research Techniques (taic part 2008), 2008, pp. 59-63. doi:10.1109/ TAIC-PART . 2008.8.

[346] R. P. Tan, S. Edwards, Evaluating automated unit testing in sulu, in: First International Conference on Software Testing, Verification, and Validation, ICST 2008, Lillehammer, Norway, April 9-11, 2008, IEEE Computer Society, 2008, pp. 62-71. doi:10.1109/ICST.2008.59.

URL https://doi.org/10.1109/ICST.2008.59 
[347] Y. Jia, M. Harman, Milu: A customizable, runtime-optimized higher order mutation testing tool for the full c language, in: Proceedings of the 3rd Testing: Academic and Industrial Conference Practice and Research Techniques (TAIC PART'08), Windsor, UK, 2008, pp. 94-98.

[348] A. Rajan, M. W. Whalen, M. Staats, M. P. E. Heimdahl, Requirements coverage as an adequacy measure for conformance testing, in: S. Liu, T. S. E. Maibaum, K. Araki (Eds.), Formal Methods and Software Engineering, 10th International Conference on Formal Engineering Methods, ICFEM 2008, Kitakyushu-City, Japan, October 27-31, 2008. Proceedings, Vol. 5256 of Lecture Notes in Computer Science, Springer, 2008, pp. 86104. doi:10.1007/978-3-540-88194-0_8. URL https://doi .org/10.1007/978-3-540-88194-0_8

[349] M. Weiglhofer, F. Wotawa, "on the fly" input output conformance verification, in: Proceedings of the IASTED International Conference on Software Engineering, SE '08, ACTA Press, Anaheim, CA, USA, 2008, pp. 286-291.

[350] C. Zhou, P. G. Frankl, Mutation testing for java database applications, in: Second International Conference on Software Testing Verification and Validation, ICST 2009, Denver, Colorado, USA, April 1-4, 2009, 2009, pp. 396-405. doi:10.1109/ICST.2009.43.

URL https://doi.org/10.1109/ICST.2009.43

[351] C. Zhou, P. G. Frankl, JDAMA: java database application mutation analyser, Softw. Test., Verif. Reliab. 21 (3) (2011) 241-263. doi:10.1002/ stvr.462.

URL http://dx.doi.org/10.1002/stvr.462

[352] R. Delamare, B. Baudry, S. Ghosh, Y. L. Traon, A test-driven approach to developing pointcut descriptors in aspectj, in: Second International Conference on Software Testing Verification and Validation, ICST 2009, Denver, Colorado, USA, April 1-4, 2009, 2009, pp. 376-385. doi:10. 
1109/ICST. 2009.41.

URL https://doi.org/10.1109/ICST.2009.41

[353] R. Delamare, B. Baudry, Y. L. Traon, Ajmutator: A tool for the mutation analysis of aspectj pointcut descriptors, in: Second International Conference on Software Testing Verification and Validation, ICST 2009, Denver, Colorado, USA, April 1-4, 2009, Workshops Proceedings, 2009, pp. 200-204. doi:10.1109/ICSTW.2009.41.

URL https://doi .org/10.1109/ICSTW .2009.41

[354] J. J. Domínguez-Jiménez, A. Estero-Botaro, I. Medina-Bulo, A framework for mutant genetic generation for WS-BPEL, in: M. Nielsen, A. Kucera, P. B. Miltersen, C. Palamidessi, P. Tuma, F. D. Valencia (Eds.), SOFSEM 2009: Theory and Practice of Computer Science, 35th Conference on Current Trends in Theory and Practice of Computer Science, Spindleruv Mlýn, Czech Republic, January 24-30, 2009. Proceedings, Vol. 5404 of Lecture Notes in Computer Science, Springer, 2009, pp. 229-240. doi:10.1007/978-3-540-95891-8_23.

URL https://doi .org/10.1007/978-3-540-95891-8_23

[355] E. G. Aydal, R. F. Paige, M. Utting, J. Woodcock, Putting formal specifications under the magnifying glass: Model-based testing for validation, in: Second International Conference on Software Testing Verification and Validation, ICST 2009, Denver, Colorado, USA, April 1-4, 2009, IEEE Computer Society, 2009, pp. 131-140. doi:10.1109/ICST.2009.20.

URL https://doi.org/10.1109/ICST .2009.20

[356] K. Dobolyi, W. Weimer, Harnessing web-based application similarities to aid in regression testing, in: ISSRE 2009, 20th International Symposium on Software Reliability Engineering, Mysuru, Karnataka, India, 16-19 November 2009, IEEE Computer Society, 2009, pp. 71-80. doi:10.1109/ISSRE.2009.18.

URL https : //doi .org/10.1109/ISSRE . 2009.18 
[357] M. Ellims, D. Ince, M. Petre, The csaw c mutation tool: Initial results, in: Testing: Academic and Industrial Conference Practice and Research Techniques - MUTATION (TAICPART-MUTATION 2007), 2007, pp. 185-192. doi:10.1109/TAIC.PART.2007.28

[358] A. Lakehal, I. Parissis, Structural coverage criteria for LUSTRE/SCADE programs, Softw. Test., Verif. Reliab. 19 (2) (2009) 133-154. doi:10. 1002/stvr.394.

URL https://doi.org/10.1002/stvr.394

[359] J. Durães, H. Madeira, Definition of software fault emulation operators: A field data study, in: 2003 International Conference on Dependable Systems and Networks (DSN 2003), 22-25 June 2003, San Francisco, CA, USA, Proceedings, IEEE Computer Society, 2003, pp. 105-114. doi:10.1109/DSN.2003.1209922

URL https://doi.org/10.1109/DSN .2003.1209922

[360] L. Madeyski, N. Radyk, Judy - a mutation testing tool for java, IET Software 4 (1) (2010) 32-42. doi:10.1049/iet-sen.2008.0038.

URL https://doi.org/10.1049/iet-sen.2008.0038

[361] R. Just, The major mutation framework: efficient and scalable mutation analysis for java, in: International Symposium on Software Testing and Analysis, ISSTA '14, San Jose, CA, USA - July 21 - 26, 2014, 2014, pp. 433-436. doi:10.1145/2610384.2628053.

URL http://doi . acm.org/10.1145/2610384.2628053

[362] P. Madiraju, A. S. Namin, Para $\mu$ - A partial and higher-order mutation tool with concurrency operators, in: Fourth IEEE International Conference on Software Testing, Verification and Validation, ICST 2012, Berlin, Germany, 21-25 March, 2011, Workshop Proceedings, 2011, pp. 351-356. doi:10.1109/ICSTW.2011.34.

URL https://doi .org/10.1109/ICSTW .2011.34 
[363] "muBPEL - A mutation testing tool for WS-BPEL", "Last Accessed May 2017" (2011).

URL "https://neptuno.uca.es/redmine/projects/sources-fm/ wiki/MuBPEL/"

[364] K. Winbladh, A. Ranganathan, Evaluating test selection strategies for end-user specified flow-based applications, in: P. Alexander, C. S. Pasareanu, J. G. Hosking (Eds.), 26th IEEE/ACM International Conference on Automated Software Engineering (ASE 2011), Lawrence, KS, USA, November 6-10, 2011, IEEE Computer Society, 2011, pp. 400-403. doi: 10.1109/ASE. 2011.6100083

URL https://doi .org/10.1109/ASE.2011.6100083

[365] A. A. Saifan, J. Dingel, J. S. Bradbury, E. Posse, Implementing and evaluating a runtime conformance checker for mobile agent systems, in: Fourth IEEE International Conference on Software Testing, Verification and Validation, ICST 2011, Berlin, Germany, March 21-25, 2011, IEEE Computer Society, 2011, pp. 269-278. doi:10.1109/ICST.2011.62.

URL https://doi.org/10.1109/ICST .2011.62

[366] R. Just, G. M. Kapfhammer, F. Schweiggert, Using conditional mutation to increase the efficiency of mutation analysis, in: Proceedings of the 6th International Workshop on Automation of Software Test, AST 2011, Waikiki, Honolulu, HI, USA, May 23-24, 2011, 2011, pp. 50-56. doi: $10.1145 / 1982595.1982606$

URL http://doi .acm.org/10.1145/1982595.1982606

[367] H. Dan, R. M. Hierons, SMT-C: A semantic mutation testing tools for C, in: Fifth IEEE International Conference on Software Testing, Verification and Validation, ICST 2012, Montreal, QC, Canada, April 17-21, 2012, 2012, pp. 654-663. doi:10.1109/ICST.2012.155.

URL https://doi .org/10.1109/ICST . 2012.155 
[368] M. Schirp," "mutation testing for ruby", "Last Accessed May 2017" (2012). URL "https://github.com/mbj/mutant"

[369] M. Kusano, C. Wang, Ccmutator: A mutation generator for concurrency constructs in multithreaded $\mathrm{C} / \mathrm{C}++$ applications, in: 2013 28th IEEE/ACM International Conference on Automated Software Engineering, ASE 2013, Silicon Valley, CA, USA, November 11-15, 2013, 2013, pp. 722-725. doi:10.1109/ASE.2013.6693142. URL https://doi.org/10.1109/ASE . 2013.6693142

[370] J. S. Bradbury, J. R. Cordy, J. Dingel, Mutation operators for concurrent java (j2se 5.0), in: Second Workshop on Mutation Analysis (Mutation 2006 - ISSRE Workshops 2006), 2006, pp. 11-11. doi:10.1109/ MUTATION.2006.10,

[371] G. M. Kapfhammer, P. McMinn, C. J. Wright, Search-based testing of relational schema integrity constraints across multiple database management systems, in: Sixth IEEE International Conference on Software Testing, Verification and Validation, ICST 2013, Luxembourg, Luxembourg, March 18-22, 2013, IEEE Computer Society, 2013, pp. 31-40. doi:10.1109/ICST.2013.47.

URL https://doi.org/10.1109/ICST.2013.47

[372] A. Bertolino, S. Daoudagh, F. Lonetti, E. Marchetti, XACMUT: XACML 2.0 mutants generator, in: Sixth IEEE International Conference on Software Testing, Verification and Validation, ICST 2013 Workshops Proceedings, Luxembourg, Luxembourg, March 18-22, 2013, IEEE Computer Society, 2013, pp. 28-33. doi:10.1109/ICSTW.2013.11.

URL https://doi .org/10.1109/ICSTW.2013.11

[373] C. Ye, H. Jacobsen, Whitening SOA testing via event exposure, IEEE Trans. Software Eng. 39 (10) (2013) 1444-1465. doi:10.1109/TSE. 2013. 20.

URL https://doi.org/10.1109/TSE.2013.20 
[374] A. Derezińska, K. Hałas, Analysis of Mutation Operators for the Python Language, Springer International Publishing, Cham, 2014, pp. 155-164. doi:10.1007/978-3-319-07013-1_15.

[375] E. Omar, S. Ghosh, D. Whitley, HOMAJ: A tool for higher order mutation testing in aspectj and java, in: Seventh IEEE International Conference on Software Testing, Verification and Validation, ICST 2014 Workshops Proceedings, March 31 - April 4, 2014, Cleveland, Ohio, USA, 2014, pp. 165-170. doi:10.1109/ICSTW.2014.19. URL https ://doi .org/10.1109/ICSTW .2014.19

[376] S. Mahajan, W. G. J. Halfond, Finding HTML presentation failures using image comparison techniques, in: I. Crnkovic, M. Chechik, P. Grünbacher (Eds.), ACM/IEEE International Conference on Automated Software Engineering, ASE '14, Vasteras, Sweden - September 15 - 19, 2014, ACM, 2014, pp. 91-96. doi:10.1145/2642937.2642966.

URL http://doi.acm.org/10.1145/2642937.2642966

[377] K. El-Fakih, A. Simão, N. Jadoon, J. C. Maldonado, On studying the effectiveness of extended finite state machine based test selection criteria in: Seventh IEEE International Conference on Software Testing, Verification and Validation, ICST 2014 Workshops Proceedings, March 31 April 4, 2014, Cleveland, Ohio, USA, IEEE Computer Society, 2014, pp. 222-229. doi:10.1109/ICSTW.2014.25.

URL https://doi.org/10.1109/ICSTW.2014.25

[378] K. Maruchi, H. Shin, M. Sakai, Mc/dc-like structural coverage criteria for function block diagrams, in: Seventh IEEE International Conference on Software Testing, Verification and Validation, ICST 2014 Workshops Proceedings, March 31 - April 4, 2014, Cleveland, Ohio, USA, IEEE Computer Society, 2014, pp. 253-259. doi:10.1109/ICSTW.2014.27.

URL https://doi.org/10.1109/ICSTW.2014.27

[379] T. A. Walsh, P. McMinn, G. M. Kapfhammer, Automatic detection of 
potential layout faults following changes to responsive web pages $(\mathrm{N})$,

in: M. B. Cohen, L. Grunske, M. Whalen (Eds.), 30th IEEE/ACM International Conference on Automated Software Engineering, ASE 2015, Lincoln, NE, USA, November 9-13, 2015, IEEE Computer Society, 2015, pp. 709-714. doi:10.1109/ASE. 2015.31.

URL https://doi.org/10.1109/ASE.2015.31

[380] R. Abreu, B. Hofer, A. Perez, F. Wotawa, Using constraints to diagnose faulty spreadsheets, Software Quality Journal 23 (2) (2015) 297-322. doi: $10.1007 / \mathrm{s} 11219-014-9236-4$

URL https://doi .org/10.1007/s11219-014-9236-4

[381] F. Belli, M. Beyazit, A. T. Endo, A. P. Mathur, A. da Silva Simão, Fault domain-based testing in imperfect situations: a heuristic approach and case studies, Software Quality Journal 23 (3) (2015) 423-452. doi:10. 1007/s11219-014-9242-6.

URL https://doi.org/10.1007/s11219-014-9242-6

[382] S. C. P. F. Fabbri, M. E. Delamaro, J. C. Maldonado, P. C. Masiero, Mutation analysis testing for finite state machines, in: 5th International Symposium on Software Reliability Engineering, ISSRE 1994, Monterey, CA, USA, November 6-9, 1994, IEEE, 1994, pp. 220-229. doi:10.1109/ ISSRE.1994.341378,

URL https://doi.org/10.1109/ISSRE.1994.341378

[383] A. Simao, A. Petrenko, J. C. Maldonado, Comparing finite state machine test coverage criteria, IET software 3 (2) (2009) 91-105.

[384] J. Guan, J. Offutt, A model-based testing technique for component-based real-time embedded systems, in: Eighth IEEE International Conference on Software Testing, Verification and Validation, ICST 2015 Workshops, Graz, Austria, April 13-17, 2015, IEEE Computer Society, 2015, pp. 1-10. doi:10.1109/ICSTW.2015.7107407.

URL https://doi .org/10.1109/ICSTW . 2015.7107407 
[385] F. S. O. Jr., K. Pattabiraman, A. Mesbah, Detecting inconsistencies in javascript MVC applications, in: A. Bertolino, G. Canfora, S. G. Elbaum (Eds.), 37th IEEE/ACM International Conference on Software Engineering, ICSE 2015, Florence, Italy, May 16-24, 2015, Volume 1, IEEE Computer Society, 2015, pp. 325-335. doi:10.1109/ICSE.2015.52.

URL https://doi.org/10.1109/ICSE.2015.52

[386] W. Krenn, R. Schlick, S. Tiran, B. Aichernig, E. Jobstl, H. Brandl, Momut::uml model-based mutation testing for uml, in: 2015 IEEE 8th International Conference on Software Testing, Verification and Validation (ICST), 2015, pp. 1-8. doi:10.1109/ICST. 2015.7102627.

[387] E. P. Enoiu, A. Causevic, D. Sundmark, P. Pettersson, A controlled experiment in testing of safety-critical embedded software, in: 2016 IEEE International Conference on Software Testing, Verification and Validation, ICST 2016, Chicago, IL, USA, April 11-15, 2016, IEEE Computer Society, 2016, pp. 1-11. doi:10.1109/ICST.2016.15

URL https://doi.org/10.1109/ICST.2016.15

[388] R. Matinnejad, S. Nejati, L. C. Briand, T. Bruckmann, Automated test suite generation for time-continuous simulink models, in: L. K. Dillon, W. Visser, L. Williams (Eds.), Proceedings of the 38th International Conference on Software Engineering, ICSE 2016, Austin, TX, USA, May 1422, 2016, ACM, 2016, pp. 595-606. doi:10.1145/2884781.2884797.

URL http://doi .acm.org/10.1145/2884781.2884797

[389] C. Henard, M. Papadakis, M. Harman, Y. Jia, Y. L. Traon, Comparing white-box and black-box test prioritization, in: L. K. Dillon, W. Visser, L. Williams (Eds.), Proceedings of the 38th International Conference on Software Engineering, ICSE 2016, Austin, TX, USA, May 14-22, 2016, ACM, 2016, pp. 523-534. doi:10.1145/2884781.2884791.

URL http://doi .acm .org/10.1145/2884781.2884791

[390] X. Devroey, G. Perrouin, P. Y. Schobbens, P. Heymans, Poster: Vibes, 
transition system mutation made easy, in: 2015 IEEE/ACM 37th IEEE International Conference on Software Engineering, Vol. 2, 2015, pp. 817818. doi:10.1109/ICSE.2015.263.

[391] A. Parsai, A. Murgia, S. Demeyer, LittleDarwin: A Feature-Rich and Extensible Mutation Testing Framework for Large and Complex Java Systems, Springer International Publishing, Cham, 2017, pp. 148-163. doi : 10.1007/978-3-319-68972-2_10

[392] G. Rothermel, R. H. Untch, C. Chu, M. J. Harrold, Test case prioritization: An empirical study, in: 1999 International Conference on Software Maintenance, ICSM 1999, Oxford, England, UK, August 30 - September 3, 1999, 1999, pp. 179-188. doi:10.1109/ICSM.1999.792604. URL https://doi.org/10.1109/ICSM.1999.792604

[393] A. S. Namin, S. Kakarla, The use of mutation in testing experiments and its sensitivity to external threats, in: Proceedings of the 20th International Symposium on Software Testing and Analysis, ISSTA 2011, Toronto, ON, Canada, July 17-21, 2011, 2011, pp. 342-352. doi:10.1145/2001420. 2001461 .

URL http://doi .acm.org/10.1145/2001420.2001461

[394] M. Kintis, M. Papadakis, A. Papadopoulos, E. Valvis, N. Malevris, Analysing and comparing the effectiveness of mutation testing tools: $\mathrm{A}$ manual study, in: 16th IEEE International Working Conference on Source Code Analysis and Manipulation, SCAM 2016, Raleigh, NC, USA, October 2-3, 2016, 2016, pp. 147-156. doi:10.1109/SCAM.2016.28. URL https : //doi.org/10.1109/SCAM . 2016.28

[395] R. V. Binder, Testing Object-oriented Systems: Models, Patterns, and Tools, Addison-Wesley Longman Publishing Co., Inc., Boston, MA, USA, 1999.

[396] W. Visser, What makes killing a mutant hard, in: Proceedings of the 
31st IEEE/ACM International Conference on Automated Software Engineering, ASE 2016, Singapore, September 3-7, 2016, 2016, pp. 39-44. doi:10.1145/2970276.2970345.

URL http://doi.acm.org/10.1145/2970276.2970345

[397] P. Ammann, M. E. Delamaro, J. Offutt, Establishing theoretical minimal sets of mutants, in: Seventh IEEE International Conference on Software Testing, Verification and Validation, ICST 2014, March 31 2014-April 4, 2014, Cleveland, Ohio, USA, 2014, pp. 21-30. doi:10.1109/ICST.2014. 13.

URL https://doi.org/10.1109/ICST.2014.13

[398] B. Kurtz, P. Ammann, M. E. Delamaro, J. Offutt, L. Deng, Mutant subsumption graphs, in: Seventh IEEE International Conference on Software Testing, Verification and Validation, ICST 2014 Workshops Proceedings, March 31 - April 4, 2014, Cleveland, Ohio, USA, 2014, pp. 176-185. doi:10.1109/ICSTW.2014.20.

URL https://doi.org/10.1109/ICSTW.2014.20

[399] A. Arcuri, L. Briand, A practical guide for using statistical tests to assess randomized algorithms in software engineering, in: ICSE, 2011, pp. 1-10. doi:10.1145/1985793.1985795. URL http://doi .acm.org/10.1145/1985793.1985795

[400] M. Harman, P. McMinn, J. T. de Souza, S. Yoo, Search Based Software Engineering: Techniques, Taxonomy, Tutorial, Springer Berlin Heidelberg, Berlin, Heidelberg, 2012, pp. 1-59. doi:10.1007/ 978-3-642-25231-0_1 URL http://dx.doi.org/10.1007/978-3-642-25231-0_1

[401] M. E. Delamaro, J. Offutt, Assessing the influence of multiple test case selection on mutation experiments, in: Seventh IEEE International Conference on Software Testing, Verification and Validation, ICST 2014 Workshops Proceedings, March 31 - April 4, 2014, Cleveland, Ohio, USA, 2014, 
pp. 171-175. doi:10.1109/ICSTW.2014.22

URL https : //doi .org/10.1109/ICSTW . 2014.22

[402] M. Kintis, M. Papadakis, A. Papadopoulos, E. Valvis, N. Malevris, Y. Le Traon, How effective mutation testing tools are? An empirical analysis of Java mutation testing tools with manual analysis and real faults, Empirical Software Engineering (EMSE)(accepted for publication). doi:10.1007/s10664-017-9582-5

[403] R. Gopinath, I. Ahmed, M. A. Alipour, C. Jensen, A. Groce, Does choice of mutation tool matter?, Software Quality Journal (2016) 1-50 doi:10. 1007/s11219-016-9317-7. URL http://dx.doi .org/10.1007/s11219-016-9317-7

[404] A. Márki, B. Lindström, Mutation tools for java, in: Proceedings of the Symposium on Applied Computing, SAC 2017, Marrakech, Morocco, April 3-7, 2017, 2017, pp. 1364-1415. doi:10.1145/3019612.3019825. URL http://doi .acm .org/10.1145/3019612.3019825

[405] A. Arcuri, L. C. Briand, Adaptive random testing: an illusion of effectiveness?, in: M. B. Dwyer, F. Tip (Eds.), Proceedings of the 20th International Symposium on Software Testing and Analysis, ISSTA 2011, Toronto, ON, Canada, July 17-21, 2011, ACM, 2011, pp. 265-275. doi: $10.1145 / 2001420.2001452$

URL http: //doi . acm . org/10.1145/2001420.2001452 